KERNFORSCHUNGSANLAGE JULICH

GESELLSCHAFT MIT BESCHRANKTER HAFTUNG Institut für Kernphysik

\title{
The Break-Up of the Deuteron and Stripping to Unbound States
}

by

G. Baur and D. Trautmann

Jül - 1252

Dezember 1975 


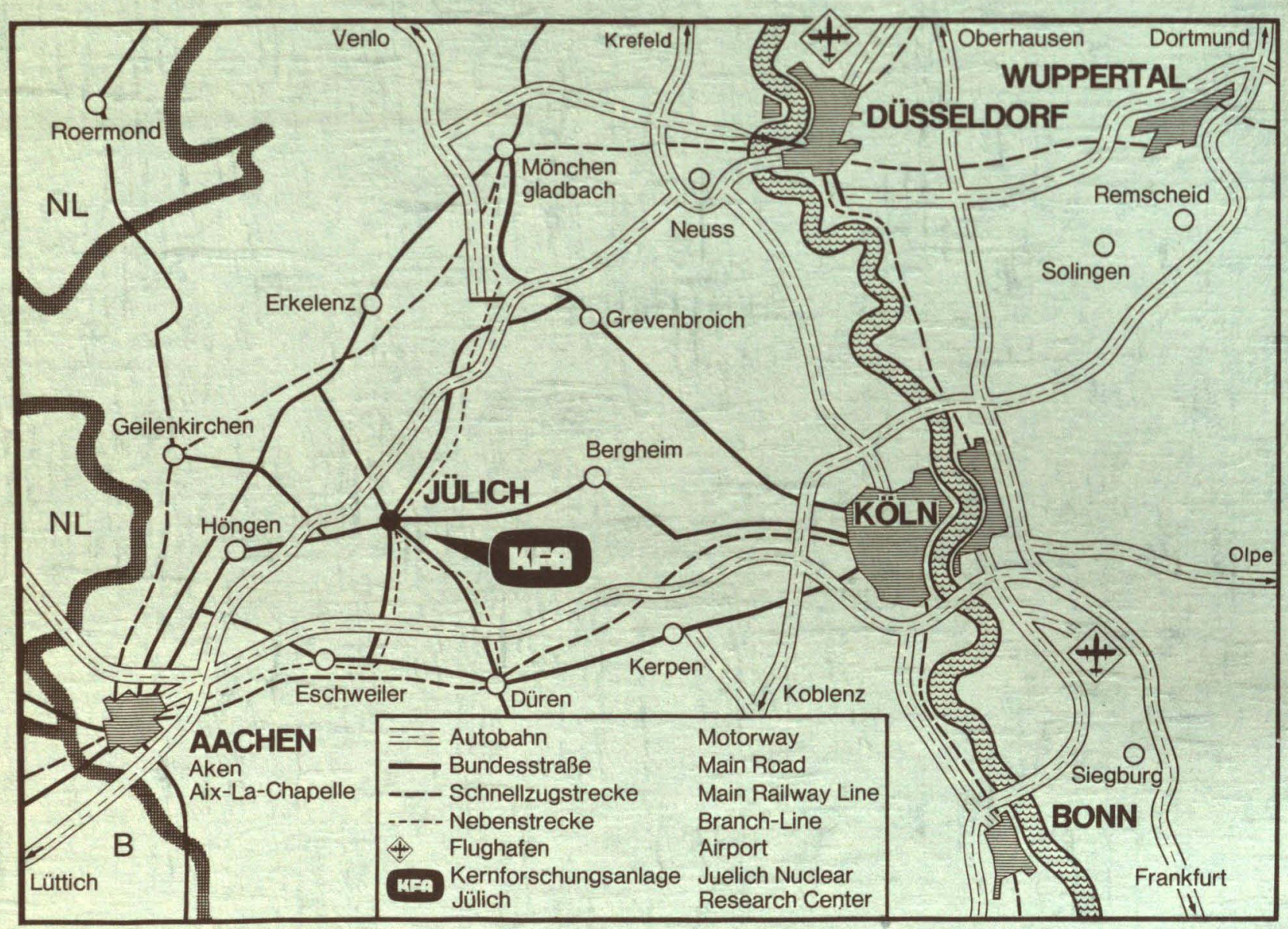

Berichte der Kernforschungsanlage Jülich - Nr. 1252

Instituł für Kernphysik Jül - 1252

Im Tausch zu beziehen durch: ZENTRALBIBLIOTHEK der Kernforschungsanlage Jülich GmbH, Jülich, Bundesrepublik Deutschland 


\title{
The Break-Up of the Deuteron and Stripping to Unbound States
}

\author{
by \\ G. Baur* and D. Trautmann**
}

* Institut für Kernphysik, Kernforschungsanlage Jülich GmbH, Jülich, Germany

** Institut für Theoretische Physik, Universität Basel, Basel, Switzerland 
List of Contents

1. Introduction

2. General Theory

2.1 Introduction

2.2 Kinematics of the Three Body Problem

2.3 Exact T-Matrix

3. DWBA Treatment of the Break-Up Reaction

3.1 DWBA-T-Matrix

3.2 Pure Coulomb T-Matrix

4. The Direct Break-Up Process

4.1 Treatment in the Prior Formalism of the DWBA

4.2 Treatment in the Post Formalism of the DWBA

4.3 Subcoulomb Deuteron Break-Up

4.4 Calculation of the Coulomb Integrals

4.5 Comparison of Subcoulomb Calculations with Experiments

5. Small Corrections

5.1 Spin Orbit Potential in the Neutron Nucleus Interaction

5.2 "Polarization" of the Deuteron in the Coulomb Field of the Nucleus

5.3 Influence of the Deuteron D-State

5.4 Compound Nuclear Effects

6. Semiclassical Model

7. Stripping into the Continuum

7.1 General Considerations

7.2 Theory

7.3 Discussion of the Model-Independent Case

7.4 Discussion of the Model-Dependent Case

7.5 Stripping to Unbound States

8. Comparison and Connection of Various Theories on Continuum Stripping Reactions

9. Conclusion and Outlook.

10. References 
Abstract

We treat the break-up of the deuteron in the field of a heavy nucleus. Our theory is based on the post formalism of the DWBA, which is discussed in detail. In particular we consider the case where the energy of the incoming deuteron is below the Coulomb barrier. This subcoulomb break-up permits a test of the DWBA by comparing experimental data with the theory. Small effects are investigated in this context, and other treatments of this process are reviewed critically. To give an intuitive picture of the break-up process we introduce a semiclassical model.

In the second part the theory is extended to stripping reactions to unbound (resonant) states. We show that this reaction which interferes with the direct break-up process, can again be well described in the post-form of the DWBA. Using simple models we discuss the most important features of such reactions. Particular attention is paid to the "l-enhancement", "parallelism" and the influence of the specific wave functions on resonance line shapes. A survey and comparison of various theories of stripping reactions to unbound states is given and finally we discuss possible future developments. 
1. Introduction

The problem of the break-up of the deuteron in the field of a nucleus is very old and basic in nuclear physics. The first speculations about this process were made by oppenheimer and Phillips $[1]$ as early as 1935. They tried to interpret the preponderance of $(d, p)$-reactions over $(d, n)$-reactions by a virtual dissociation of the deuteron in the coulomb field of the nucleus before the actual nuclear interaction takes place. Because of the Coulomb repulsion of the proton this would explain the afore mentioned dominance of $(d, p)$-reactions. In this context, oppenheimer $[2]$ also tried to treat the real break-up of the deuteron in the coulomb field of a nucleus.

It was not before the important work by Butler $[3]$ that stripping reactions became accessible to theoretical treatment. Later on Butler's theory was superseded by the more complicated distorted-wave-born-approximation (DWBA), yielding a quantitative description of stripping reactions to bound states. (For a detailed and excellent review of this topic, see e.g. ref. [4]). In most treatments up to now the influence of the actual breakup of the deuteron on stripping reactions has been neglected. There are theoretical arguments $[5]$ that it is a small effect, but the problem actually involves all the complications of a three-body rearrangement collision. In an approximate way Johnson andSoper have shown $[6]$ how to include virtual break-up effects in the deuteron optical model potential. 
The question of the real break-up of the deuteron, on the other hand, has been considered in an important paper by Landau and Lifshitz $[7]$. They used boundary condition methods, similar to the ones introduced later on by Butler $[3]$. The result obtained by these authors is equivalent to the zero-range-DWBA result. Their paper has been critically discussed by Breit $[8]$. Some numerical results based on the formulae of Landau and Lifshitz have been reported by Ketchum $[9] ;$ extensive investigations of the corresponding DWBA expressions were given by the present authors $[10]$. The appealing result of Landau and Lifshitz was also rederived by Butler and Austern [11] .

The ideas of Landau and Lifshitz did not receive the attention which they deserved. Instead, theorists pursued different ways of investigation. Attempts were made to treat the deuteron break-up in a perturbation approach which completely neglected the fact that the center-of-mass of the proton-neutron system in the final state is not moving on a Rutherford trajectory. The first paper along these lines by Mullin and Guth $[12]$ appeared in 1951. They considered the deuteron break-up as a Coulomb excitation process into the continuum. In 1963, more extensive numerical calculations, based on the same model, were made by Gold and wong $[13]$. During that time numerous experiments on the deuteron break-up reaction were also performed. After the early measurements of the $(d, p)$-reaction in the medium energy region by Cohen and Falk $[14]$ and Aschenbrenner $[15]$, extensive coincidence measurements of the deuteron break-up reaction were performed by the Pittsburgh group $[16-18]$ by Udo and coworkers $[19-21]$ and by Nemets et al. $[22]$. Later, very 
accurate coincidence measurements were performed by J. Lang, L. Jarczyk et al. $[23-26]$.

The accuracy of these measurements allows to test the different existing theories. In 1972, Rybicky and Austern $[27]$ compared extensive numerical calculations based on their theory, which incorporates nuclear interactions in the model of Mullin and Guth $[12]$, with experiment. Finding no agreement they interpreted the failure as a consequence of the poor description of the final state.

In this work we want to describe a post-interaction DWBA approach to the problem of deuteron break-up, which overcomes these short-comings and is able to quantitatively explain the experiments. This report is based on (and in part reviews) our earlier investigations $[28-32]$. Furthermore, we study small corrections, like the deuteron D-state, "polarization", and spinorbit interactions, which may be important in the break-up reaction. Particularly instructive is the "subcoulomb" breakup, where the energy of the incoming deuteron is below the coulomb barrier. In this case ambiguities due to optical model parameters are absent and hence the investigation of the breakup process allows for a clean test of the DWBA. Furthermore, the semiclassical limit can be derived and thus an intuitive picture of the process as well as an accurate approximation are provided. Our approach to the break-up reaction lends itself very easily to the more particular problem of stripping reactions to unbound states. Indeed, in the experimental study of transfer reactions not only the usual type of stripping re- 
actions to bound states is observed (see e.g. ref. $[4]$ ), but occasionally resonant states are also populated. Early observations of this type were made by Alty et al. [33]. A particularly careful study of transfer reactions into the continuum, measuring resonant as well as background contributions, was made by Fuchs et al. $[34,35]$. They established an interesting relation between transfer reactions to unbound states and the elastic scattering of neutrons.

Theoretically, the problem of stripping reactions to unbound states was first studied by Friedmann and Tobocman [36], who modified the old Butler theory of stripping reactions to treat the case of stripping to unbound states also. We will show that this simple theory can explain many experimentally observed features of such reactions $[34,35,37]$. Further progress was made by Huby and Mines $[38]$, and later on by Vincent [39]. They solved the convergence problem of the postform expression of the DWBA. A formal discussion of this DWBA expression was given by Levin $[40,4.1]$ and a complex integration metheod to calculate numerically the slowly convergent radial integrals occurring in this DWBA treatment was described by vincent and Fortune $[42]$. Their powerful method allows an easy calculation of stripping reactions to unbound states. On the other hand, different models were introduced into the theory of continuum stripping reactions in order to avoid the calculation of these slowly convergent integrals, namely the theories developed by Bang and zymany $[43]$, by coker $[44]$, by Bunakov et al $[45-48]$, by Schlessinger and Payne $[49]$, by Lipperheide and Möhring $[50-52]$, 
by Noble $[53]$, by Sharaf $[54]$ and by Dolinsky et al. $[55-57]$. A short review of all these theories is given in this paper.

Although it is possible to analyze the experimental data in the framework of the DWBA by using the integration technique of Vincent and Fortune $[58-60]$, it does not give an easy insight to the physical mechanism of such transfer reactions into the continuum. Nevertheless, in some special cases intuitive interpretation is possible. Therefore, we will discuss here in particular the subcoulomb case and the plane-wave limit which still contain most of the interesting features of such processes.

Finally, we will discuss some of the possible future experimental and theoretical investigations.

\section{General Theory}

\section{2.=1_Introoㅁuㅡ늠으}

The process we want to describe is of the type

$$
d+A \rightarrow p+n+A
$$

where a particle d (typically a deuteron) upon hitting the target nucleus A breaks up into a particle $\mathrm{p}$ (proton) and a particle $n$ (neutron). We are mostly interested in the special case where $\mathrm{A}$ remains unexcited, i.e. we do not ascribe any internal structure to $A$. 
One can think of various mechanisms leading to this final state. Firstly, the proton-target and the neutron-target interaction, characterized by optical potentials $V_{p A}$ and $V_{n A}$ respectively, can directly lead to a disintegration of the deuteron. This process, which we want to call direct break-up, will be the subject of chapter 4. Secondly, we can also imagine that either the proton or the neutron, or even the deuteron, will form a resonant state with the target nucleus (resonances that may not be accounted for by the energy averaged optical potentials $V_{p}$ and $V_{n A}$ mentioned above). The subsequent decay of the resonance will then again lead to the three-body final state $p+n+A$ of eq. (2.1), i.e.

$$
d+\mathrm{A} \longrightarrow \begin{aligned}
& (n+A)_{\text {res }}+p \\
& (p+A)_{\text {res }}+n \longrightarrow \\
& (d+A)_{\text {res }}
\end{aligned} \longrightarrow p+n+A
$$

It is worth considering the energetics of the various reaction mechanisms of eq. (2.2) : for a given incident deuteron energy $E_{d}$ the resonant states ${ }^{(n+A)}$ res and $(p+A)$ res can be reached by sharing the total energy between the outgoing proton or neutron. Thus a sharp energy $E_{p}$ (within the width of the resonance) is associated with the formation of the resonance in the neutron chanel $(n+A)$, and a sharp energy $E_{n}$ corresponds to the formation of a resonance in the channel $(p+A)$. Note that in this case the energy of the emitted proton will be spread out due to recoil effects. The $(d+A)$ res state can of course only be formed for a selected energy $E_{d}$. In this case, both the neutron and proton final 
state energies will be spread out due to recoil effects. Of course, all these processes cannot be distinguished from each other. The various amplitudes will have to be added coherently.

In general, the resonant states can also decay into other fragments than $A+p+n$. This means that one has to consider the internal structure of A.

In the following, special emphasis is put on deuteron and heavy ion induced reactions with energies comparable to the coulomb barrier.

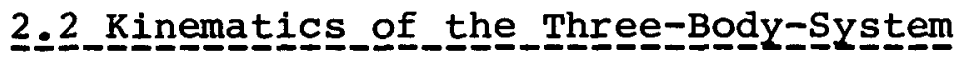

Since the reactions we want to describe are characterized by a three-body final state, we want to review briefly the pertinent kinematics (for further information we refer the reader to the literature, see e.g. ref. $[61$, pg $550 \mathrm{ff.}]$ or ref. $[62]$ ). The "canonical"parameters are introduced as shown in fig. 1 . (Hereby we use a notation which is suggestive of an $A(d, p n) A$ reaction, though, of course, $d=p+n$ may denote any particle).

The masses of the three particles are denoted by $m_{A}, m_{P}$ and $m_{n}$, their coordinates in an arbitrary frame of reference are given by $\vec{R}_{A}, \vec{R}_{p}$ and $\vec{R}_{n}$, their momenta by $\vec{p}_{A}, \vec{p}_{p}$ and $\vec{p}_{n}$. The total kinetic energy is given by

$$
T_{\text {kin }}=\frac{p_{A}^{2}}{2 m_{A}}+\frac{p_{p}^{2}}{2 m_{p}}+\frac{p_{n}^{2}}{2 m_{n}} \text {. }
$$


In the center of mass frame which is defined by

$$
m_{A} \vec{R}_{A}+m_{p} \vec{R}_{p}+m_{n} \vec{R}_{n}=0
$$

we have

$$
\vec{p}_{p}+\vec{p}_{p}+\vec{p}_{n}=0
$$

Let us now consider the subsystem $p+n$ and describe the motion of A with respect to the center of mass of this subsystem. We introduce the relative vector between particles $i$ and $j$ by $\vec{x}_{i j}=\vec{R}_{j}-\vec{R}_{i}$ and the distance of particle $k$ form the center of mass of particles $i$ and $j$ by

$$
\vec{\rho}_{k}=-\frac{M}{m_{i}+m_{j}} \vec{R}_{k}
$$

where

$$
M=m_{i}+m_{j}+m_{k}
$$

The momentum conjugate to $\vec{\rho}_{k}$ is then given by

$$
\vec{Q}_{k}=\mu_{k} \dot{\vec{\rho}}_{k}=-m_{k} \vec{R}_{k}=-\vec{p}_{k}
$$

with the reduced mass

$$
\mu_{k}=\frac{m_{k}\left(m_{i}+m_{j}\right)}{M}
$$

The momentum conjugate to $\vec{X}_{i j}$ is given by 
$\vec{p}_{i j}=m_{i j} \dot{\overrightarrow{x_{i j}}}=\frac{m_{i} \vec{p}_{j}-m_{j} \vec{p}_{i}}{m_{i}+m_{j}}$,

with

$$
m_{i j}=\frac{m_{i} m_{j}}{m_{i}+m_{j}}
$$

To describe the initial bound state of $p+n(=d)$, we introduce the convenient coordinates $\vec{x}_{n p}$ and $\vec{\rho}_{p}$ together with their conjugate momenta. The kinetic energy in the center of mass system is then given by

$$
T_{k i n}=\frac{Q_{A}^{2}}{2 \mu_{A}}+\frac{p_{n p}^{2}}{2 m_{n p}},
$$

In the exit channel we choose the coordinates $\vec{x}_{n A}$ and ${\overrightarrow{S_{p}}}_{p}$. The kinetic energy is then written as

$$
T_{k i n}=\frac{p_{n A}^{2}}{2 m_{h A}}+\frac{Q_{p}^{2}}{2 \mu_{p}},
$$

where

$$
\vec{p}_{n A}=\frac{m_{A} M}{\left(m_{p}+m_{A}\right)\left(m_{n}+m_{A}\right)} \vec{Q}_{n}+\frac{m_{n}}{m_{n}+m_{A}} \vec{p}_{p A} .
$$

Alternatively, we could have chosen the coordinates $\vec{x}_{p A}$ and $\vec{\rho}_{n}$. In this case we have

$$
T_{k, n}=\frac{Q_{n}^{2}}{2 \mu_{n}}+\frac{p_{p A}^{2}}{2 m_{p A}}
$$

These kinematical relations allow us to calculate the phase space factor $\rho$ (phase) (the "density of final states") which enters into the quantum mechanical expression for the cross section : 
$\frac{d^{3} \sigma}{d \Omega_{p} d \Omega_{n} d E_{n}}=2 \pi \cdot \frac{\mu_{d}}{q_{d}} \quad \rho$ (phase) $\left.|| T_{f i}\right|^{2}$,

where $T_{f i}$ is the corresponding T-matrix element. We assume that the plane-wave solution of the wave functions entering into the T-matrix is normalized as

$$
\Psi_{\vec{p}}^{(s)}(\vec{r})=U_{\sigma}^{s} e^{i \vec{p} \vec{r}},
$$

where $U_{\sigma}^{s}$ is a spinor of rank $s$ (the spin). Then we have the following normalization :

$$
\left\langle\Psi_{\vec{p}}^{(s)}, \Psi_{\vec{p}^{\prime}}^{\left(s^{\prime}\right)}\right\rangle=(2 \pi)^{3} \delta_{s, s^{\prime}} \cdot \delta\left(\vec{p}-\vec{p}^{\prime}\right)
$$

The space factor $\rho_{\text {(phase) }}$ can be evaluated by using the fact that the total number of states for the three final particles is given by $d^{3} q_{a} d^{3} p_{n p} / h^{6}$. Following ref. $[62]$, and writing the phase space factor as the following integral

$$
\begin{aligned}
\rho_{(\text {phase })}\left(E_{n}\right) d E_{n} d \Omega_{n} d \Omega_{p} & =\frac{1}{h^{6}} \int d^{3} p_{A} d^{3} p_{p} d^{3} p_{n} . \\
& \delta\left(\vec{p}_{-} \vec{p}_{p}-\vec{p}_{n}-\vec{p}_{p}\right) \cdot \delta\left(E-E_{n}-E_{n}-E_{p}\right),
\end{aligned}
$$

where $\vec{P}$ is the total momentum (=0 in the center of mass system) and $\mathrm{E}$ is the total available kinetic energy, we easily get :

$\rho_{(\text {phose) }}\left(E_{n}\right) d E_{n} d \Omega_{n} d \Omega_{p}=\frac{h^{-6} m_{A} m_{n} m_{P} p_{p} p_{n}}{\left(m_{A}+m_{n}\right)+\frac{m_{n}\left(\vec{p}_{p}-\vec{p}\right) \vec{p}_{n}}{p_{n}^{2}}} d E_{n} d \Omega_{n} d \Omega_{p}$. 
For the case $m_{A} \gg m_{n}, m_{p}$ to be considered in the following the phase space factor can safely be approximated by

$$
\rho(\text { phase }) \approx \frac{\mu_{p} m_{n}}{h^{6}} q_{p} q_{n}
$$

In this asymmetric case it becomes more convenient to introduce other coordinates, $\vec{r}_{p A}=-\vec{x}_{p A}, \vec{r}_{n A}=-\vec{x}_{n A}, \vec{r}_{d A}=\vec{\rho}_{A}$ and $\vec{r}_{n p}=-\vec{x}_{n p}$. For the momenta we write $\vec{q}_{d}=\vec{Q}_{A}, \vec{q}_{n}=\vec{p}_{n A}$ and $\vec{q}_{p}=-\vec{Q}_{p}$.

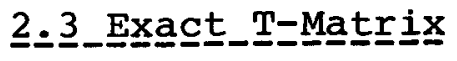

We idealize the problem of the break-up reaction to a three particle problem and write the total Hamiltonian in the following way :

$$
H=T_{p}+T_{n}+T_{A}+V_{n A}\left(\vec{r}_{n A}\right)+V_{p A}\left(\vec{r}_{p A}\right)+V_{n p}\left(\vec{r}_{n p}\right)
$$

where $T_{P}, T_{n}$ and $T_{A}$ denote the kinetic energy operator of $\mathrm{p}, \mathrm{n}$ and $\mathrm{A}$, respectively. The neutron-proton interaction $V_{n p}\left(\vec{r}_{n p}\right)$ is well known for our purposes in the low energy region. In the triplett state there exists a bound-state - the deuteron with a binding energy of $B_{d} \equiv \frac{\hbar^{2} \alpha^{2}}{2 m_{n p}}=2.2246 \mathrm{MeV}$. This defines the quantity $\alpha$ used later on. The neutron- and protontarget interactions $V_{n A}\left(\vec{r}_{n A}\right)$ and $V_{p A}\left(\vec{r}_{p n}\right)$ are given by their appropriate optical potentials.

As a side remark we mention here that effects due to the proper antisymmetrization of the target- and projectile-nucleons (not considered in our model) have been studied in various papers by Gambhir and Griffin $[63-65]$. These so-called "Pauli break-up" 
terms seem to be "neither large nor unusual" (see ref. $[66]$ ).

The free Hamiltonians of the deuteron- and of the break-up channel (in- and outgoing channel) are given by

$$
H_{i}=T_{k i n}+V_{n p}
$$

and

$$
H_{f}=T_{\text {kin }}
$$

where $T_{k i n}$ is total kinetic energy.

The initial and final state interactions are given by

$$
V_{i}=H-H_{i}=V_{n A}+V_{p A}
$$

and

$$
V_{f}=H-H_{f}=V_{n A}+V_{p A}+V_{n p} .
$$

According to Gerjuoy $[67]$ the transition matrix element in the "prior representation" is given by

$$
\left.T_{f i}^{(}\right)=\left\langle\Psi_{f}^{()}\left|V_{i}\right| \varphi_{d}\right\rangle,
$$

where $\Psi_{f}^{()}$is the full solution of the Schrödinger equation with only ingoing neutron and proton spherical waves.

$\Psi_{f}^{()}$can be defined by $[39]:$

$$
\Psi_{f}^{()}=\varphi_{f}+\frac{1}{E-i \varepsilon-H} V_{f} \varphi_{f},
$$

with

$$
\varphi_{f}=e^{i\left(\vec{q}_{n} \vec{r}_{n A}+\vec{q}_{P} \vec{r}_{P A}\right)}
$$

In the following part of this chapter, $A$ is assumed to be infinitely heavy. 
The wave function $\varphi_{d}$ denotes a free deuteron incident in direction $\vec{q}_{d}:$

$$
\varphi_{d}=\Phi_{d}\left(\vec{r}_{n p}\right) e^{i \vec{q}_{d} \vec{r}_{d A}}
$$

where $\Phi_{d}\left(\vec{r}_{n p}\right)$ is the internal deuteron wave function.

The corresponding "post representation" of the T-matrix is formally written as

$$
T_{f i}^{(+)}=\left\langle\varphi_{f}\left|V_{f}\right| \Psi_{i}^{(+)}\right\rangle,
$$

where $\Psi_{i}^{(+)}$is again the full solution of the schrödinger equation with the boundary condition for an outgoing deuteron. It was argued by Gerjuoy [67] that this expression is divergent and therefore should not be used. Later on, however, vincent $[39]$ introduced an exact formulation of the post-expression by taking the following limit :

$$
T_{f i}^{(t)}=\lim _{\varepsilon \rightarrow 0+}\left\langle\chi_{f}^{(-)}\left|e^{-\varepsilon r_{n p}} V_{n p}\left(\vec{r}_{n p}\right)\right| \Psi_{i}^{(t)}\right\rangle .
$$

This exact expression may be regarded as an interpretation of the non-convergent form

$$
T_{f i}^{(+)}=\left\langle\chi_{f}^{(-)}\left|V_{n p}\right| \Psi_{i}^{(+)}\right\rangle,
$$


which results from a purely formal derivation. (For the derivation of eq. (2.30) we refer the reader to the literature $[39]$ ).

By deriving eq. (2.30) the Gell-Mann-Goldberger relations were used (cf.ref $[68]$ ) to replace the full interaction in the breakup channel, $V_{f}=V_{n p}+V_{n A}+V_{p A}$, by $V_{n p}$. The remaining potentials $V_{p A}+V_{n A}$ were used to "distort" $\varphi_{f}$ into $\chi_{f}^{()}$:

$$
\chi_{f}^{(-)}=\varphi_{f}+\frac{1}{E-i \varepsilon-\tilde{H}_{f}}\left(V_{p D}+V_{n A}\right) \varphi_{f},
$$

which is an exact solution of the Hamiltonian

$$
\tilde{H}_{f}=T_{k i n}+V_{p A}+V_{n A} \quad, \tilde{V}_{f}=H-\tilde{H}_{f}=V_{n p} .
$$

A detailed and more suggestive derivation of eq. (2.31) with the help of wave packets is given in ref. $[4]$.

Naturally, the Gell-Mann-Goldberger relation can also be applied to the "prior-representation". For this reason a potential $V_{d A}$ acting only on the center of mass of the neutron-proton system, is introduced. This potential gives rise only to elastic deuteron scattering, and leads to

$$
T_{f i}^{(-)}=\left\langle\Psi_{f}^{(-)}\left|\tilde{V}_{i}\right| \chi_{i}^{(t)}\right\rangle
$$

where $\chi_{i}^{(t)}$ describes a deuteron, whose center of mass motion is given by a distorted wave in the potential $V_{d A}$. Thus $\chi_{i}^{(t)}$ is a solution of the initial state Hamiltonian 


$$
\tilde{H}_{i}=T_{k i n}+V_{d A}+V_{n p}, \tilde{V}_{l}=H-\widetilde{H}_{i}=V_{p A}+V_{n A}-V_{d A}
$$

We note here that the derivation of the prior form presents less difficulties than the corresponding derivation of the "post-type" transition matrix elements, since in the former one no divergence problems arise. However, as we will see later, the post-form is much more useful for practical purposes. This is due to the fact that $V_{n p}$ is of shorter range than $V_{p A}+V_{n A}-V_{d A}$

\section{DWBA Treatment of the Break-Up Reaction}

The computation of the exact T-matrices eqs. (2.30) or (2.34) requires a treatment of the full quantal three-body problem. There is an exact theory available, the Faddeev theory; however, this method is very hard to realize in practice. This leads us to consider the DWBA theory of stripping, which has successfully been passing the test of stripping experiments to bound states during the last two decades.

\section{$\underline{3}=\underline{1}$ DWBA-T-Matr}

In the DWBA the exact solution $\Psi_{i}^{(+)}$, which enters into the post-form matrix element (2.31), is approximated by the distorted wave $\chi_{i}^{(t)}$ introduced previously. In general, this approximation can be expected to be good if inelastic processes are weak*, i.e.

* Possible rescattering effects, which are not included in the DWBA, were studied by Friedman $[69]$. 


$$
\Psi_{i}^{(t)} \approx \chi_{i}^{(t)}\left(\vec{r}_{n p}, \vec{r}_{d A}\right)=\chi_{\vec{q}_{d}}^{(t)}\left(\vec{r}_{d A}\right) \Phi_{d}\left(\vec{r}_{n p}\right)
$$

A similar approximation, whose quality depends on the weakness of $V_{n p}$ in the break-up channel, can be introduced in the prior form of the DWBA*

$$
\Psi_{f}^{(-)} \approx \chi_{f}^{(-)}\left(\vec{r}_{p A}, \vec{r}_{n A}\right)=\chi_{\vec{q}_{p}}^{(-)}\left(\vec{r}_{p A}\right) \chi_{\vec{q}_{n}}^{(-)}\left(\vec{r}_{n A}\right) .
$$

This leads to the prior form of the DWBA. It can be shown $[38,39]$ that the post and prior form of the DWBA are identical, i.e.

$$
T_{f i}^{(-)}(D W B A)=T_{f i}^{(+)}(D W B A)
$$

with * *

$$
T_{f i}^{(-)}(D W B A)=\lim _{\varepsilon \rightarrow 0^{+}}\left\langle\chi_{f}^{(-)}\left|e^{-\varepsilon r_{n A}} V_{n p}\right| \chi_{i}^{(t)}\right\rangle
$$

and

$$
T_{f i}^{(t)}(D W B A)=\left\langle\chi_{f}^{\leftrightarrow)}\left|V_{n A}+V_{p A}-V_{d A}\right| \chi_{i}^{(t)}\right\rangle .
$$

Quite analogous to the case of bound state stripping, the usual definition of the optical potential $V_{p A}$ leads to terms in addition to $V_{n p}$ (see ref. $[4, \mathrm{pg} .159 \mathrm{ff}$.$] ). We assume that these$ correction terms are negligibly small.

If $A$ is not considered infinitely heavy the difference between $\vec{r}_{p A}$ and $-\vec{\rho}_{p}$ leads to correction terms of the order of $1 / A$, which are neglected in the following. 
From a historical point of view it is worth noticing here that this equivalence was first stated by Huby and Mines $[38]$ in 1965. They performed a partial wave decomposition of the prior DWBA matrix element $T_{f i}^{(-)} *$ ) and showed that each partial wave matrix element is identical to a corresponding partial wave matrix element in the post representation. Thus, in their treatment, convergence of the post-form matrix element was introduced not by a limiting process, but rather by a special order of the integration which is implied by the partial wave decomposition.

After having established the DWBA amplitudes $(3.4,3.5)$ it is a technical problem to calculate these T-matrices. In practice this means that we have to introduce a partial wave expansion for the distorted waves which may be written as

$$
\chi_{\vec{q} \sigma}^{(t)}(\vec{r})=\sum_{\sigma^{\prime}} \chi_{\sigma^{\prime}, \sigma}^{(t)}(\vec{q}, \vec{r}) U_{\sigma^{\prime}}^{s},
$$

where

$$
\begin{gathered}
\chi_{\sigma^{\prime}, \sigma}^{(t)}(\vec{q}, \vec{r})=\frac{4 \pi}{q r} \sum_{\substack{j l \mu \\
m m^{\prime}}} i^{l}\langle l m s \sigma \mid j \mu\rangle\left\langle l m^{\prime} s \sigma^{\prime} \mid j \mu\right\rangle \\
Y_{l m}^{*}(\hat{q}) Y_{l m^{\prime}}(\hat{r}) \chi_{j l}(q, r) .
\end{gathered}
$$

Here we have restricted our consideration to optical potentials which conserve orbital angular momentun. Eq.(3.7) satisfies the time reversal relation 
$-20-$

$$
\chi_{\sigma^{\prime}, \sigma}^{(-)}(\vec{q}, \vec{r})=(\rightarrow)^{\sigma-\sigma^{\prime}} \chi_{-\sigma^{\prime}-\sigma^{(+)}}^{(-\vec{q}, \vec{r})} .
$$

In the case of no spin-orbit interaction eq. (3.6) reduces to

$$
X_{\vec{q} \sigma}^{(+)}(\vec{r})=X_{\vec{q}}^{(+)}(\vec{r}) U_{\sigma}^{s}=\frac{4 \pi}{q r} \sum_{l m} i^{l} Y_{l m}^{*}(\hat{q}) Y_{l m}(\hat{r}) X_{l}(q, r) U_{\sigma}^{s}
$$

where the asymptotic form of $\chi_{l}(q, r)$ is given by

$$
X_{l}(q, r) \sim \frac{i}{2} e^{i \sigma_{l}(\eta)}\left\{H_{l}^{*}(\eta, q r)-S_{l} \cdot H_{l}(\eta, q r)\right\} .
$$

The $S_{L}$ are the usual s-matrix elements and $H_{l}(\eta, q r)$ is related to the regular and irregular coulomb functions $F_{l}(\eta, q r)$ and $G_{l}(\eta, q r)$ by

$$
H_{l}(\eta, q r)=G_{l}(\eta, q r)+i F_{l}(\eta, q r)
$$

The coulomb parameter $\eta$ is defined by

$$
\eta_{k}=\frac{z e^{2} \mu_{k}}{\hbar^{2} q_{k}}
$$

while $\sigma_{l}(\eta)=\arg \Gamma(l+1+i \eta)$ is the coulomb phase shift and $z$ denotes the product of the charges of the two colliding particles. In general, we use the same expansion (3.7) for the outgoing newtron. We then will denote the radial neutron wave function by

$$
f_{j i}(q, r)=\frac{\chi_{j l}(q, r)}{q r}
$$


In the special case, however, where the neutron is captured in a resonance state $B=(A+n)$ res' a more general neutron wave function has to be used, namely $[40]$ :

$$
\begin{aligned}
& \chi_{\vec{q}_{n}}^{(-)}\left(\vec{r}_{n A} ; J_{A}, M_{A}, \nu_{f}\right)=4 \pi \sum i^{l}\left\langle J_{A} M_{A} j^{\prime} \mu^{\prime} \mid J_{B} M_{B}\right\rangle\left\langle l^{\prime} m \frac{1}{2} \nu_{f} \mid j^{\prime} \mu^{\prime}\right\rangle . \\
& \sum_{l^{\prime} m^{\prime} J_{B} j_{B}^{\prime} \mu_{B}^{\prime} \bar{M}_{A}} \cdot\left\langle\operatorname{lm} \frac{1}{2} \nu l_{j \mu}\right\rangle\left\langle J_{A} \bar{M}_{A} j \mu \mid J_{B} M_{B}\right\rangle \text {. } \\
& \text { - } Y_{l m}\left(\hat{r}_{n A}\right) Y_{l^{\prime} m^{\prime}}^{*}\left(\hat{q}_{n}\right) U_{\nu}^{\frac{1}{2}} \\
& \Phi_{\bar{H}_{A}}^{J_{A}} \cdot f_{j j^{\prime}, l l^{\prime}}^{J_{B^{\prime}} J_{A}}\left(q_{n}, r_{n A}\right),
\end{aligned}
$$

where now the wave function $f_{j j^{\prime}, L^{\prime}}^{J_{B}, J_{A}}\left(q_{n}, r_{n A}\right)$ has to be determined by a coupled-channel calculation. The internal wave function of the core $A$, which is assumed to have spin and magnetic quantum numbers $J_{A}$ and $\bar{M}_{A}$ is denoted by $\Phi_{\bar{M}_{A}}^{J_{A}}$. of course, in this case, we also have to introduce the wave function $\Phi_{\hat{M}_{A}}^{J_{A}}$, describing $A$ in its initial state, into the $T$-matrix and to integrate over the internal coordinates of $\mathrm{A}$.

The internal wave function of the deuteron is given by

$$
\Phi_{d}^{s_{d} m_{d}}\left(\vec{r}_{n p}\right)=\sum_{J L} i^{L} u_{J L}\left(r_{n p}\right) y_{L s_{d} s_{d}^{\prime}}^{m_{d}},
$$

where $(J, L)=\left\{\left(\frac{1}{2}, 0\right) ;\left(\frac{3}{2}, 2\right)\right\}$ and $s_{d}=S_{d}^{\prime}-1$ and

$$
\eta_{l s j}^{\mu}=\sum_{m \sigma}\left\langle\left\lfloor m s \sigma|j \mu\rangle Y_{l m}(\hat{r}) U_{\sigma}^{s} .\right.\right.
$$

of course, the deuteron wave function can be represented in a different basis, more convenient for the description of other projectiles : 


$$
\begin{aligned}
\Phi_{d}^{s d, m d}\left(\vec{r}_{n p}\right)=\sum_{J L} i^{L} u_{J L}\left(r_{n p}\right) \sum_{\substack{M \mu_{d} \\
\sigma_{p} \sigma_{n}}} & \left\langle\frac{1}{2} \sigma_{n} L M \mid J \mu d\right\rangle Y_{L M}\left(\hat{r}_{n p}\right) \\
& \cdot\left\langle J \mu_{d} \frac{1}{2} \sigma_{p} \mid s_{d} m_{d}\right\rangle U_{\sigma_{n}}^{\frac{1}{2}} U_{\sigma_{p}}^{\frac{1}{2}} .
\end{aligned}
$$

The radial deuteron wave functions $u_{J L}(r)$ behave like $e^{-\alpha r} / r$ for $r \rightarrow \infty$, with $\alpha=0.2317 \mathrm{fm}^{-1}$. Several choices for these radial wave functions are known in the literature. In most cases we will use the wave functions proposed by Hulthen and Sugawara $[70]$ :

$$
u_{\frac{1}{2} 0}(r)=N \cos \varepsilon_{g} \frac{e^{-\alpha r}}{r}\left(1-e^{-\mu_{0}\left(r-r_{c}\right)}\right) \theta\left(r-r_{c}\right),
$$

and

$$
u_{\frac{3}{2} 2}(r)=N \sin \varepsilon_{g} \frac{e^{-\alpha r}}{r}\left(1-e^{-\mu_{2}\left(r-r_{c}\right)}\right)^{2}\left(1+\frac{3\left(1-e^{-\mu_{2} r}\right)}{\alpha r}+\frac{3\left(1-e^{-\mu_{2} r}\right)^{2}}{\alpha^{2} r^{2}}\right) \cdot \theta\left(r-r_{c}\right)
$$

where we have used the step-function, defined by

$$
\theta(x)=\begin{array}{ll}
0 & x<0 \\
1 & x \geqslant 0
\end{array}
$$

The normalization constant $\mathrm{N}$ is related to the effective range St obtained from neutron-proton scattering :

$$
N=\sqrt{2 \alpha}\left(1-\alpha \rho_{t}\right)^{-\frac{1}{2}}
$$

and $\varepsilon_{g}$ is the mixing parameter in the deuteron ground state. The parameters $\mu_{0}, \mu_{2}$ and $\varepsilon_{g}$ are connected with the hard core radius $r_{c}$, the D-state probability $P_{D}$ and the effective 
range $\rho_{t}$. For the corresponding values see ref. $[70]$.

In the special case where we are only considering the S-state of the deuteron $\left(\varepsilon_{g}=0\right)$ we will choose eq. (3.18a) with $r_{c}=0, \quad \mu_{0} \approx 5.39 \alpha$ and

$$
N \cos \varepsilon_{g}=N_{H}=\left(\frac{2 \alpha \beta(\beta+\alpha)}{\mu_{0}^{2}}\right)^{\frac{1}{2}}, \beta=\alpha+\mu_{0} \text {. }
$$

Inserting all these wave functions into the DWBA expressions eqs. $(3.4,3.5)$ yields the general T-matrix element :

$$
\begin{aligned}
& T_{f i}^{( \pm)}=4 \pi \sum i^{L-l_{n}}\left\langle\frac{1}{2} \sigma_{n} L M \mid J \mu_{d}\right\rangle\left\langle J \mu_{d} \frac{1}{2} \sigma_{p} \mid s_{d} m_{d}\right\rangle \\
& \underset{j L}{J_{L} \sigma_{n} \mu_{d} m_{d} M}\left\langle J_{A} \hat{M}_{A} j_{n}^{\prime} \mu_{n}^{\prime} \mu_{n}^{\prime} \mid J_{B} M_{B}\right\rangle\left\langle\ln _{n}^{\prime} m_{n}^{\prime} \frac{1}{2} \nu_{f} \mid j_{n}^{\prime} \mu_{n}^{\prime}\right\rangle \\
& \ln _{J_{B} m_{n} \ln _{B}^{\prime} m_{n}^{\prime}}\left\langle J_{A} M_{A} j_{n} \mu_{n} \mid J_{B} M_{B}\right\rangle\left\langle l_{n} m_{n} \frac{1}{2} \sigma_{n} \mid j n \mu_{n}\right\rangle \\
& Y_{l n m_{n}^{\prime}}\left(\hat{q}_{n}\right) t_{f i}^{( \pm)}\left(L M ; J_{B} J_{A} j n j_{n}^{\prime} \ln \ln ^{\prime} m_{n}\right),
\end{aligned}
$$

with

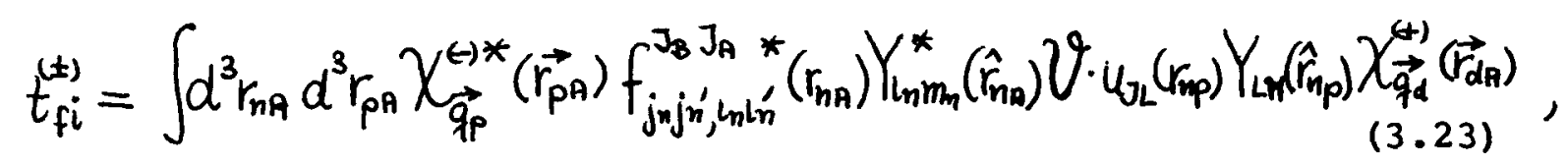

where we have neglected spin orbit interactions in the optical potentials and where $V$ denotes the corresponding potentials occurring in the post or prior T-matrix element, respectively.

All observable quantities can be expressed in terms of these matrix elements, e.g. the cross section where the neutron is not observed is given by : 


$$
\begin{aligned}
& \frac{d^{2} \sigma}{d \Omega_{p} d E_{n}}=\frac{\mu_{d} \mu_{p} m_{n}}{2\left(\pi \hbar^{2}\right)^{3}} \frac{q_{p}}{q_{\alpha}} q_{n} \frac{1}{2 J_{A}+1} \sum_{J L}\left(2 J_{B}+1\right)(2 k+1) . \\
& J_{B} j n j_{n}^{\prime} \ln k \\
& \left|\sum_{L M_{m}}(-)^{m_{n}}\left\{\begin{array}{lll}
l_{n} & L & k \\
J & j_{n} & \frac{1}{2}
\end{array}\right\}\left(\begin{array}{ccc}
l_{n} & L & k \\
m_{n}-M & k
\end{array}\right) t_{f i}^{( \pm)}\left(L M_{j} J_{B} J_{A} j_{n} j_{n}^{\prime} l_{n} m_{n}\right)\right|^{2},
\end{aligned}
$$

where we have used the approximate phase space factor (2.21). Equivalent formulae were given in ref. [40] (c.f. the analogous expressions used in bound state stripping reactions $[4,71,72]$ ).

After the principal problem of the convergence of the postform matrix element has been removed, there still remains the difficult numerical problem of calculating the occurring radial integrals in the partial wave decomposition. The radial integrals are, in the zero-range approximation, of the type

$$
R_{l d l n l p}=\int_{0}^{\infty} d r \chi_{l d}\left(q_{d}, r\right) f_{j n l_{n}}\left(q_{n}, r\right) \chi_{l_{p}}\left(q_{p}, r\right) .
$$

Due to the asymptotic behaviour (3.10) of the wave functions, convergence is ensured by the oscillations at infinity. But numerically, this convergence is not attained even at upper integration limits of, say, $400 \mathrm{fm}$. Huby and Mines proposed [38] to introduce a convergence factor $e^{-E r}$ into the radial integrals (3.25) and to evaluate the limit $R_{L d \ln l p}(\varepsilon \rightarrow 0)$. However, this method turns out to be rather impractical. Many calculations of $R_{l_{d} l_{n} l_{p}}(\varepsilon)$ with rather small values of $\varepsilon$ are needed in order to obtain a reliable extrapolation to $\varepsilon \rightarrow 0$. The much more ele- 
gant complex integration method was introduced by vincent and Fortune $[42]$. In their method the range of integration is divided into an inner part $0 \leqslant r \leqslant r_{t}$ and an outer part $r_{t} \leqslant r<\infty$ with $r_{t}$ chosen such that the nuclear potentials become negligible in the outer part. The integral over the inner range is evaluated by a standard program, while in the outer range the asymptotic wave function $\chi_{\ell_{\alpha}}$ is split into $H^{*}$ and $H$ parts. These parts can be integrated along a line parallel to the imaginary axis. Due to the kinematical condition

$$
q_{d}>q_{p}+q_{n},
$$

following from energy conservation, this integrand converges rapidly in this direction. Using Cauchy's integral theorem, it can be shown furthermore that the integral then can be completed by a circle with infinite radius giving a vanishing contribution. Since there are no contributions from poles of the integral in the outer region, the evaluation of the whole integral can therefore be done quite easily, and the post-form of the DWBA can be used for our further analysis.

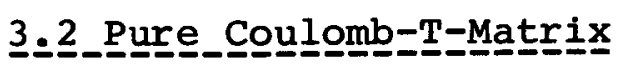

The case of the sub-Coulomb deuteron break-up is especially clean and important. By definition sub-Coulomb means here that the optical model wave function of the deuteron incident on the target A may be replaced by a pure Coulomb wave function 


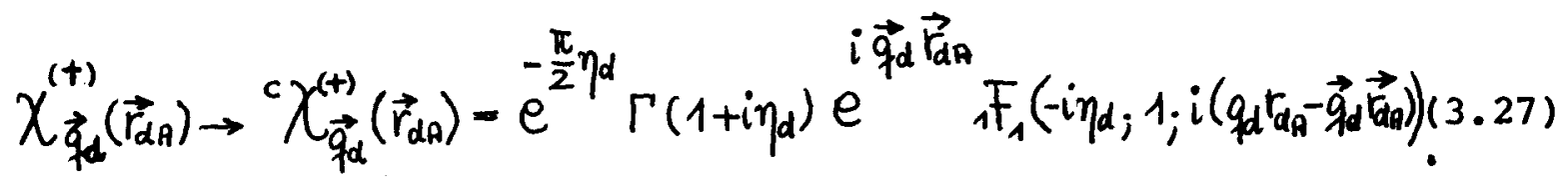

This occurs when the energy of the incoming deuteron is below a "safe bombarding energy" which is slightly smaller than the usual energy of the Coulomb barrier. This choice of the energy of the deuteron implies that the wave function of the outgoing proton may be, a fortiori, replaced by the corresponding coulomb function $\quad \chi_{\vec{q}_{P}}^{(-)}\left(\vec{r}_{P A}\right)$. This is justified because the proton energy is always smaller than $E_{d}-B_{d}$. This fact is very important for the discussion of sub-coulomb break-up reactions, compared to bound state stripping, where the mostly positive Q-value $\left(\equiv E_{p}-E_{d}\right)$ of the reaction leads to a nuclear distortion in the proton channel, even at small bombarding energies. Unfortunately, the same arguments cannot be used for the outgoing neutron, i.e. its wave function can never be replaced by a plane wave. Nevertheless, we will define a so-called pure coulomb T-matrix $[10]$ by using a plane wave for the neutron as

$$
T_{p c}=\left\langle\chi_{\vec{q}_{p}}^{(-)} e^{i \vec{q}_{n} \cdot \vec{r}_{n A}}\left|V_{n p}\left(r_{n p}\right)\right| \phi_{d}\left(\vec{r}_{n p}\right) \chi_{\vec{q}_{d}}^{c}\left(\vec{r}_{d A}\right)\right\rangle
$$

which is, in the zero range approximation (c.f. eq. (4.10)), approximated by

$$
T_{p} c=D_{0}\left\langle{ }^{c} x_{\vec{q}_{p}^{(-)}}^{(\vec{r})} e^{i \overrightarrow{q_{n} \cdot \vec{r}}}\right|{ }^{c} x_{\left.\vec{q}_{d}^{(+)}(\vec{r})\right\rangle=D_{0} M_{p} c .} .
$$

The introduction of this "model T-matrix" will be useful in our further investigations. 
4. The Direct Break-Up Process

As mentioned above, we mean by a direct break-up the process where the deuteron disintegrates directly under the influence of the nuclear optical model potentials (including the Coulomb part)

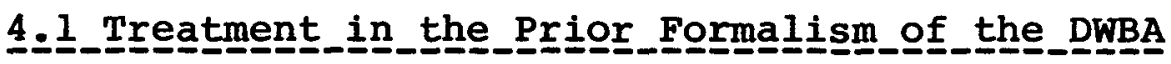

In the prior form of the DWBA we have to calculate the matrix element (3.5). In order to illustrate the difficulties in the evaluation of this matrix element, let us take a simple example : we neglect the neutron-target interaction $V_{n A}$ and restrict ourselves to pure coulomb distortions in the deuteron-target and proton-target interaction. We obtain the following matrix element ("pure Coulomb break-up") :

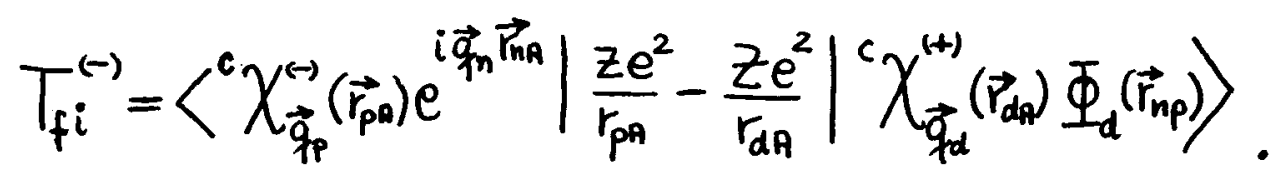

We attempt to evaluate this six-dimensional integral by choosing $\vec{\gamma}_{p p}$ and $\vec{r}_{d A}$ as integration variables. All other choices seem less favourable, because it would involve using addition theorems for coulomb (or even optical model) wave functions. Now we express $\vec{r}_{n A}$ and $\vec{r}_{n p}$ in terms of $\vec{r}_{p A}$ and $\vec{r}_{d A}$ :

$$
\vec{r}_{n A}=\vec{r}_{p A}+\vec{r}_{n p} \quad \text { and } \quad \vec{r}_{n p}=2\left(\vec{r}_{d A}-\vec{r}_{p A}\right)
$$


(where we have chosen $m_{A} \gg m_{n}$ for simplicity's sake). The neutron plane wave can most easily be expressed in our integration variables. By choosing a suitable form of the deuteron wave function $(e .9$. a Hulthèn wave function for an s-state deuteron) a convenient expansion in the integration coordinates $\vec{r}_{p A}$ and $\vec{r}_{d A}$ can be found. The final formula in partial wave decomposition is given by :

$$
\begin{aligned}
& T_{f i}^{(H)}=8 \cdot N_{H}(4 \pi)^{\frac{5}{2}} \sum_{\substack{l_{d} l_{p} l_{n} \\
l_{1} l_{2} \lambda}} i^{i l_{p}-L_{d}+l_{1}+l_{2}} e^{i\left(\sigma_{u}\left(\eta_{\alpha}\right)+\sigma_{p}\left(\eta_{p}\right)\right)} \hat{l}_{d} \hat{l}_{p} \hat{l}_{n} \hat{l}_{1}^{2} \hat{l}_{2}^{2} \hat{\lambda}^{2} \text {. }
\end{aligned}
$$

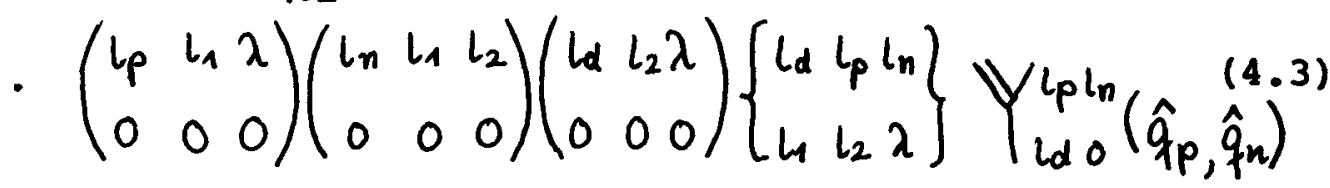

$$
\begin{aligned}
& \text { - }\left(R_{l_{d} l_{p} l_{n}}^{l_{1} l_{2} \lambda}(\alpha)-R_{l_{d} l_{p} l_{n}}^{l_{1} l_{2} \lambda}(\beta)\right) \text {. }
\end{aligned}
$$

Here we have introduced the tensor product of two shperical harmonics (so-called bipolar harmonics) by

$$
\begin{aligned}
Y_{l d m_{d}}^{l_{p} l_{n}}\left(\hat{q}_{p}, \hat{q}_{n}\right) & \equiv\left[Y_{l_{p} m_{p}}\left(\hat{q}_{p}\right) Y_{l_{n} m_{n}}\left(\hat{q}_{n}\right)\right]_{l_{d} m_{d}} \\
& =\sum_{m_{p} m_{n}}\left\langle l_{p} m_{p} l_{n} m_{n} \mid l_{d} m_{d}\right\rangle Y_{l_{p} m_{p}}\left(\hat{q}_{p}\right) Y_{l n m_{n}}\left(\hat{q}_{n}\right) .
\end{aligned}
$$

Using the incident-beam coordinate system, in which the z-axis is defined by the direction of $\vec{q}_{d}$ and the y-axis by $\vec{q}_{d} \times \vec{q}_{p}$ we have

$$
Y_{L_{d} m_{d}}\left(\hat{q}_{d}\right)=\sqrt{\frac{2 L_{d}+1}{4 \pi}} \delta_{m_{d}, 0}
$$

and therefore 


$$
\begin{aligned}
Y_{l d m_{d}}^{l_{p} l_{n}}\left(\hat{q}_{p}, \hat{q}_{n}\right) \rightarrow Y_{l_{d} 0}^{l_{p} l_{n}}\left(\hat{q}_{p}, \hat{q}_{n}\right)=\sum_{m_{n}}\left\langle l_{p}-m_{n} l_{n} m_{n} \mid l_{d} 0\right\rangle . \\
Y_{l_{p}, m_{n}}\left(\theta_{p}, 0\right) Y_{l_{n} m_{n}}\left(\theta_{n}, \Phi\right),
\end{aligned}
$$

where $\theta_{p}$ and $\theta_{n}$ are the scattering angles of proton and neutron in the center of mass system and $\Phi$ is the difference between their azimuth angles.

The radial integrals in eq. (4.3) are defined by

$$
\begin{aligned}
& R_{l_{d} l_{p} l_{n}}^{l_{1} l_{2} \lambda}(\alpha)=\frac{1}{q_{d q_{p}}} \int_{0}^{\infty} d r_{d A} j_{l_{2}}\left(2 q_{n} r_{d A}\right) F_{l_{d}}\left(\eta_{d}, q_{d} r_{d A}\right) \text {. } \\
& \left\{h_{\lambda}^{(t)}\left(2 i \alpha r_{d A}\right) \int_{0}^{r_{\alpha A}} d r_{p A} j_{\lambda}\left(2 i \alpha r_{p A}\right)\left(r_{d A}-r_{P A}\right) j_{l_{1}}\left(q_{n} r_{P A}\right) F_{\varphi_{P}}\left(\eta_{P}, q_{P} r_{P A}\right)\right. \\
& \left.+j_{\lambda}\left(2 i \alpha r_{d A}\right) \int_{r_{d A}}^{\infty} d r_{P A} h_{\lambda}^{(+)}\left(2 i \alpha r_{p A}\right)\left(r_{d A}-r_{p A}\right) j_{l_{1}}\left(q_{n} r_{p A}\right) F_{L_{p}}\left(\eta_{p}, q_{P} r_{p A}\right)\right\} .
\end{aligned}
$$

The second integral in eq. (4.3), defined as eq. (4.7) but with $\alpha$ replaced by $\beta$, leads to a very small angular dependent finiterange correction and can therefore be neglected.

In our numerical attempts we managed to calculate the radial integrals reasonably accurately and fast, but the ensuing sums over the $l$-values turned out to be fraught with difficulties. Partial cancellation effects show up, and the convergence is extremely poor. We definitely considered such a calculation as too time-consuming to be practicable. We want to note that the introduction of "distortion" into the neutron wave function can be handled in a simple way only in the region outside the nuclear interaction (where it is also most important); there a Sawaguri-Tobocman-type of addition theorem $[73]$ is available, leading to an expression quite similar to eq. (4.3). 
To overcome the difficulties by calculating the Coulomb part of the prior amplitude two approximations have been introduced in the literature $[12,13,27]$ : firstly a multipole expansion for the potential difference in (4.1) is used, giving

$$
\begin{aligned}
& P\left(\vec{r}_{n p}, \vec{r}_{d R}\right) \equiv Z e^{2}\left\{\frac{1}{r_{p A}}-\frac{1}{r_{d A}}\right\}=Z e^{2}\left\{\frac{1}{\left|\vec{r}_{d A}-\frac{1}{2} \vec{r}_{n p}\right|}-\frac{1}{r_{d A}}\right\}= \\
& =Z e^{2}\left\{\theta\left(r_{n p}-2 r_{d A}\right)\left(\frac{2}{r_{n p}}-\frac{1}{r_{d A}}\right)\right. \\
& \left.+4 \pi \sum_{l=1}^{\infty} \sum_{m=-l}^{l} \frac{1}{2 l+1}\left(\frac{\theta\left(2 r_{d A}-r_{n p}\right)}{r_{d A}^{l+1}}\left(\frac{r_{n p}}{2}\right)^{l}+\frac{\theta\left(r_{m p}-2 r_{d A}\right)}{\left(r_{m p} / 2\right)^{l+1}} r_{d A}^{l}\right) Y_{l m}^{*}\left(\hat{r}_{d A}\right) Y_{l m}\left(\hat{r}_{n p}\right)\right\} .
\end{aligned}
$$
Expression $(4.8)$ can be approximated, using $\bar{r}_{n p} \ll \bar{r}_{d \theta}$, by

$$
P\left(\vec{r}_{n p}, \vec{r}_{d \theta}\right) \simeq Z e^{2} \frac{2 \pi}{3} \sum_{\mu=-1}^{+1} \frac{r_{n p}}{r_{d A}^{2}} Y_{1 \mu}^{*}\left(\hat{r}_{d p}\right) Y_{1 \mu}\left(\hat{r}_{n p}\right) .
$$

This so-called "dipole approximation" will be well fulfilled because the main contribution to the radial integral comes, due to the short range character of the neutron-proton interaction, from $r_{d A} \gg r_{n p}$. Naturally, one can also take into account higher terms in eq. (4.8).

The second and more serious approximation consists of replacing the DWBA final state in eq. (4.1) by $\chi_{\overrightarrow{q_{\alpha}}}^{(-)}\left(\vec{r}_{d A}\right) \cdot \Phi_{d}^{()}\left(\vec{r}_{n p}\right)$, where $\chi_{\vec{q}_{\alpha}}^{\leftrightarrow}\left(\vec{r}_{d A}\right)$ is now an optical wave function for the unbound final deuteron moving in the field of the target nucleus and $\Phi_{d}^{\leftrightarrow}\left(\vec{r}_{n p}\right)$ a neutron-proton scattering wave function (to be used with incoming wave boundary conditions). This wave function may be interpreted as a Coulomb excitation of the initial bound state of the deuteron into an unbound scattering state $\Phi_{d}^{(-)}\left(\vec{r}_{n p}\right)$.

Introducing both approximations in the prior matrix element (4.1) we get for the pure coulomb break-up an expression which is very similar to the one given by Mullin and Guth in their 
perturbation treatment [12]. This approach was followed by Gold and wong [13]. They calculated numerous examples of break-up cross-sections. The matrix-elements occurring in their treatment are well known in the theory of Coulomb excitztion [74]. Ribicky and Austern $[27]$ refined the prior treatment of the break-up process by taking into acount all coulomb and nuslear interactions exactly. Again, the radial integrals occurring in Coulomb excitation theory can be used to speed up the numerical calculation. Although the authors were able to show that the nuclear interactions are very important and are never negligible they found even in their refined model no agreement between theory and experiment, especially for low deuteron energies. They explain this disagreement by the bad description of the finzl state.

Indeed, using this prescription the nuclear and coulomb field of the target nucleus act on the center of mass of the neutronproton system in the final state, which is surely a bad approximation : while the theoretical results are symmetric in the neutron and proton energy, the corresponding experimental values show a pronounced asymmetry.

Finally, we conclude that either the numerleal treatment of the prior form of the DWBA, using eqs. $(4.3-4.7)$, is very cumbersome or the approximations used to make such a calculation feasible are too poor to be acceptatic. 


\section{4._2_Treatment_in_the_Post_Formalism_of_the_DWBA}

In the post-form of the DWBA the T-matrix we have to calculate is given by eq. (3.4) .

In order to calculate the corresponding matrix element in stripping reactions to bound states it is customary to use the socalled zero-range approximation.:

$$
V_{n p}\left(\vec{r}_{n p}\right) \Phi_{d}\left(\vec{r}_{n p}\right) \simeq D_{0} \delta\left(\vec{r}_{n p}\right)
$$

where the zero-range constant $D_{0} \simeq-123.5 \mathrm{MeV} \mathrm{fm}^{3 / 2}$ is related to the asymptotic normalization $\mathbf{N}$ of the deuteron wave function by

$$
D_{0}=\frac{N}{\sqrt{2 \alpha}} D_{0}^{0} \quad \text { with } \quad D_{0}^{0}=-\frac{\hbar^{2}}{2 m_{m p}} \sqrt{8 \pi \alpha} \text {. }
$$

By using this zero-range approximation we get for the T-matrix

$$
T_{f i}^{(t)}=D_{0} \int d^{3} r \chi_{\vec{q}_{p}}^{(-) *}(\vec{r}) \chi_{\vec{q}_{n}^{*}}^{(t)}(\vec{r}) \chi_{\vec{q}_{d}}^{(t)}(\vec{r}),
$$

where the integral has to be understood in the sense discussed above.

Neglecting all spin-orbit interactions in the nuclear distortion we get by using (3.9) :

$$
\begin{array}{r}
T_{f i}^{(t)}=D_{0}(4 \pi)^{2} \sum_{l_{d} l_{n} l_{p}} i^{l_{d}+l_{n}+l_{p}} e^{i\left(\sigma_{l_{d}}(\eta d)+\sigma_{L_{p}}\left(\eta_{p}\right)\right)} \hat{l}_{d} \hat{l}_{n} \hat{l}_{p} \\
\left(\begin{array}{ccc}
l_{d} & \ln l_{p} \\
0 & 0 & 0
\end{array}\right) Y_{l_{d} 0}^{l_{p} l_{n}}\left(\hat{q}_{p}, \hat{q}_{n}\right) R_{l_{d} \ln l_{p},}^{n u c},
\end{array}
$$

with the radial integral over the nuclear optical model wave functions of deuteron, neutron and proton, respectively : 


$$
R_{l_{d} l_{n} l_{p}}^{n u c}=\int_{0}^{\infty} d r \chi_{l_{d}}\left(q_{d}, r\right) f_{l_{n}}\left(q_{n}, r\right) \chi_{l_{p}}\left(q_{p}, r\right) .
$$

These radial integrals can be calculated by using the integration technique of Vincent and Fortune described above. Nevertheless, due to the large numbers of angular momenta contributing to the sum the calculation of (4.13) would be quite cumbersome. The computing time can, however, be greatly reduced if the T-matrix is split up in the following way :

$$
\begin{array}{r}
T_{f i}^{(+)}=D_{0}\left\{M_{p c}+(4 \pi)^{2} \sum_{l_{d} l_{n} l_{p}} i^{l_{d}+l_{n}+l_{p}} \hat{l}_{d} \hat{l}_{n} \hat{l}_{p} e^{i\left(\sigma_{l_{d}}\left(\eta_{d}\right)+\sigma_{l p}\left(\eta_{p}\right)\right)}\right. \\
\left.\left(\begin{array}{ccc}
l_{d} & \ln l_{p} \\
0 & 0 & 0
\end{array}\right) Y_{l_{d} 0}^{l_{p} l_{n}}\left(\hat{q}_{p}, \hat{q}_{n}\right)\left(R_{l_{d} \ln l_{p}}^{n u c}-R_{l_{d} \ln l_{p}}^{0}\right)\right\}_{,}^{(4.15)}
\end{array}
$$

where the radial integrals $R_{\text {ld }}^{0} \ln l_{p}$ are given by

$$
R_{l d l n}^{0}=\frac{1}{q_{\alpha} q_{p}} \int_{0}^{\infty} d r F_{l_{\alpha}}\left(\eta_{d}, q_{\alpha} r\right) j_{l_{n}}\left(q_{n} r\right) F_{l_{p}}\left(\eta_{p}, q_{p} r\right) .
$$

If $l_{x}\left(=l_{d}, l_{n}\right.$ or $\left.l_{p}\right)$ is larger than $l_{x}^{\max }(\simeq 10)$ then the optical model functions $\chi_{l_{x}}$ are pure coulomb or Bessel functions, respectively. Therefore, the terms in the sum in eq. (4.15) vanish and we are left with the calculation of the "pure coulomb term" $M_{p c}$ defined in eq. (3.29). This matrix element also appears in the theory of the bremsstrahlung process and was expressed by Sommerfeld $[75]$ and Nordsieck $[76]$ in terms of hypergeometric functions and is given by $[7,9,10,25,26]$ :

$$
M_{p c}=e^{-\frac{\pi}{2}\left(\eta_{d}+\eta_{p}\right)} \Gamma\left(1+i \eta_{d}\right) \Gamma\left(1+i \eta_{p}\right) I_{p c},
$$


with

$$
\begin{aligned}
I_{p c}= & -i \lim _{\lambda \rightarrow 0+} \frac{d}{d \lambda}\left\{B(\lambda){\underset{2}{1}}_{1}\left(-i \eta_{d},-i \eta_{p} ; 1_{j} \xi(\lambda)\right)\right\} \\
=-i & \left\{B(0)\left(\frac{d \xi(\lambda)}{d \lambda}\right)_{\lambda=0}\left(-\eta_{d} \eta_{p}\right){ }_{2} F_{1}\left(1-i \eta_{d}, 1-i \eta_{p} ; 2 ; \xi(0)\right)\right. \\
& \left.+\left(\frac{d B(\lambda)}{d \lambda}\right)_{\lambda=0}{ }_{2} F_{1}\left(-i \eta_{d},-i \eta_{p} ; 1 ; \xi(0)\right)\right\},
\end{aligned}
$$

where we have used the following definitions :

$$
\begin{gathered}
B(\lambda)=\frac{4 \pi}{q^{2\left(i \eta_{\alpha}+i \eta_{p}+1\right)}}\left(q^{2}-2 \vec{q} \vec{q}_{\alpha}-2 \lambda q_{\alpha}\right)^{i \eta_{d}}\left(q^{2}+2 \vec{q} \vec{q}_{\alpha}-2 \lambda q_{p}\right)^{i \eta_{p}}, \\
\vec{q}=\vec{q}_{\alpha}-\vec{q}_{p}-\vec{q}_{n},
\end{gathered}
$$

and

$$
\xi(\lambda)=2 \cdot \frac{q^{2}\left(q_{d} q_{p}+\vec{q}_{d} \overrightarrow{q_{p}}\right)-2\left(\vec{q} \vec{q}_{d}+\lambda q_{d}\right)\left(\overrightarrow{q_{q}} \vec{q}_{p}-\lambda q_{p}\right)}{\left(q^{2}-2 \vec{q} \vec{q}_{d}-2 \lambda q_{\alpha}\right)\left(q^{2}+2 \vec{q} \vec{q}_{p}-2 \lambda q_{p}\right)} .
$$

Since $|\xi(0)|$ is for many actual cases greater than unity, a suitable analytic continuation of the hypergeometric function has then to be used $[77]$. (Note that $f(0)<0$ ).

As mentioned above, expression (4.17) is - multiplied by $D_{0}$ just the expression given by Landau and Lifshitz in their treatment of the direct break-up reaction $[7]$ (cf. also the critical discussion of this work in ref. [8]). In those days computers were not so widespread. Therefore, those authors gave suitable approximations for the different possible cross sections obtainable from eq. (4.17) which may be valuable for an estimate of the cross section. Using a saddle point approximation for the hypergeometric functions in (4.18) valid for $\eta_{d}, \eta_{p} \gg 1$ we have 
according to Landau and Lifshitz :

$\frac{d^{3} \sigma}{d \Omega_{p} d \Omega_{n} d E_{n}}=D_{0}^{2} \cdot \frac{\alpha \eta_{d}^{3} E_{n}^{1 / 2} S_{2} S_{3}}{B_{d}^{2} \pi^{2} 2^{1 / 2} E_{d}^{3 / 2}} e^{-\eta_{d} S}$

where we have used the following abbreviations (changing the authors' notation) :

$$
S=s_{0}+s_{1} \frac{E_{n}}{E_{d}}+s_{2} \frac{E_{n}}{E_{d}} \sin ^{2} \theta_{n}+s_{3} \theta_{p}^{2}+s_{4} \theta_{p} \sqrt{E_{n}} \sin \theta_{n} \cos \Phi, \text { (4.22) }
$$

with

$$
s_{0}=\sqrt{\frac{8}{1-v}} \arctan \sqrt{\frac{2 v}{1-v}}-4 \arctan \sqrt{v} \text {, where } v=\frac{B_{d}}{E_{d}} \text {, }
$$

and

$$
\begin{aligned}
& S_{1}=\frac{\sqrt{2}}{(1-v)^{2 / 2}} \arctan \sqrt{\frac{2 v}{1-v}}-\frac{2 \sqrt{v}(1-3 v)}{(1+v)^{2}(1-v)}, \\
& S_{2}=\frac{4 \sqrt{v}}{(1+v)^{2}}, \quad s_{3}=\frac{\sqrt{v}}{(\sqrt{2}+\sqrt{1-v})^{2}}, \\
& S_{4}=\frac{4 \sqrt{v}}{(1+v)(\sqrt{2}+\sqrt{1-v})}
\end{aligned}
$$

Integrating over the angles we get :

$$
\frac{d \sigma}{d \Omega_{p}}=\sigma_{\text {tot }} \eta_{d} \cdot \frac{s_{1} s_{3}}{\pi\left(s_{1}+s_{2}\right)} \exp \left\{-\eta_{d} \theta_{p}^{2} \frac{s_{1} s_{3}}{s_{1}+s_{2}}\right\},
$$


and for the energy distribution of the neutron :

$$
\frac{d \sigma}{d E_{n}}=\sigma_{\text {tot }} \frac{2}{\sqrt{\pi}}\left(\frac{\eta_{\alpha}}{E_{\alpha}} s_{1}\right)^{\frac{3}{2}} \sqrt{E_{n}} e^{-\frac{\eta_{d}}{E_{d}} E_{n} s_{1}}
$$

and where the total cross section is given by

$$
\sigma_{t o t}=D_{0}^{2} \frac{\alpha \sqrt{\pi_{\eta \alpha}} s_{2}}{B_{d}^{2} 2^{9 / 2} s_{1}^{3 / 2}} e^{-\eta_{\alpha} s_{0}} .
$$

Tables for the total cross section are given in ref. $[8]$. Although our calculations showed that the approximations (4.21 4.29) are only fulfilled within $10 \%$ to 508 in general, they display the correct dependence on the physical parameters, e.g. we obtain from eq. (4.28) for the energy of the neutron, where the cross section is maximal :

$$
E_{n}^{o p t}=\frac{E_{d}}{2 \eta d S_{1}}
$$

Furthermore, we have the possibility to estimate the order of magnitude of the break-up cross section due to the coulomb field of the nucleus.

In the neighbourhood of the Coulomb barrier a simple estimate of the influence of the nuclear distortions on the break-up cross section is possible by using the so-called diffraction model [4]. Applying this model we replace the nuclear wave functions in the radial integrals $(4.14)$ by their asymptotic values and simulate the 
nuclear distortion in the deuteron and proton channel by introducing the factor $\sqrt{a_{l d} a_{l_{p}}}$ in the partial wave sum (4.15). According to the strong absorption model $[78,79]$ we write

$$
a_{l}=\left(1+i \tau \frac{\partial}{\partial L}\right) \otimes\left(1+\exp \left(\frac{l_{0}-L}{\Delta}\right)\right)^{-1},
$$

where $l$ stands for $l_{d}$ and $l_{p}$ respectively. The parameters $\tau, l_{0}$ and $\Delta$ may be related in this model to the physical parameters entering in the reaction (e.g. Lo can be related to the angle $\theta_{c}$, where the deuteron or proton elastic scattering cross section deviates from the pure Rutherford cross section by the relation $l_{0}=\eta \operatorname{ctg} \frac{\theta_{c}}{2}$ ). Since, however, it is not clear whether the deuteron or even the proton is a strongly absorbing particle $[4]$ we will use this model only for an estimate of the nuclear distortions. (Nevertheless, it was shown in ref. [29] that the theoretical results based on this model are in very good agreement with experiment). Naturally, a careful treatment of the break-up reaction above the coulomb barrier, calculating eq. (4.15), should use optical model wave functions extracted from elastic scattering experiments.. Such calculations were performed by Lang et al. $[25]$, who use optical potentials of the usual Woods-saxon form :

$$
u(r)=u_{C}(r)-v_{0} f\left(x_{0}\right)-i\left(4 w_{D} \frac{d f\left(x_{D}\right)}{d x_{D}}+w f\left(x_{w}\right)\right),
$$

with

$$
f\left(x_{i}\right)=\left(1+e^{x_{i}}\right)^{-1}, \quad x_{i}=\frac{r-r_{i} A^{1 / 3}}{a_{i}},
$$


and where $U_{c}$ is the well-known coulomb potential of a uniformly charged sphere. The deuteron optical model parameters they used were the ones given by Perey and Perey [80] and for proton and neutron they used parameters given by Wilmore and Hodgson [81] . Calculations along the same lines were done in ref. $[82]$, where also energies far above the coulomb barrier were considered. The calculations at energies close to the Coulomb barrier show very good agreement with experiments presented in ref. $[25]$. Even at higher energies (17 MeV-deuterons incident on $\mathrm{Pb}$ ) the agreement between experiment and theory is quite satisfactory, as can be seen in fig. 2 in a typical case. In these calculations effects of non-locality of the optical potentials were taken into account just in the same way as has usually been done for bound state stripping reactions, i.e.every scattering wave function occurring in the radial integral (4.14) is multiplied with the corresponding non-local correction function (see ref. $[4, \mathrm{pg} .114]$ ). However, it turned out that this correction is completely negligible in the energy region of interest. On the other hand, including finite range effects by multiplying the integrand in (4.14) with the finite range correction function $[83,4]$

$$
\Lambda(r)=\left\{1-\frac{\alpha^{2}}{\beta^{2} B_{d}}\left(V_{P A}(r)+V_{n A}(r)-V_{d A}(r)+B_{d}\right)\right\}^{-1}
$$

gives a small but non-negligible correction, leading to an even better agreement between experiment and theory. Furthermore, these investigations show that the break-up cross section is 
quite sensitive to the optical model parameters which have been used, particularly to those of the neutron. Keeping the well-known optical model parameter ambiguities in mind it is therefore necessary to perform experiments in the sub-Coulomb energy region to have a clean test of the DWBA approach to break-up reactions.

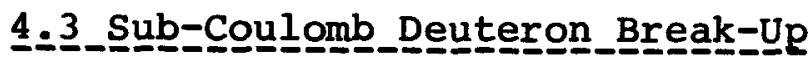

As was discussed before, the case where the energy of the incoming deuteron is below the Coulomb barrier is of special interest since this sub-Coulomb break-up allows a clean test of the reaction mechanism.

It is well known from sub-Coulomb stripping reactions to bound states that the contributions coming from the nuclear interior are largely supressed and we are therefore allowed to use only the asymptotic scattering wave functions, i.e. Coulomb wave functions $[72]$. Furthermore, we claim that we can also use the asymptotic neutron scattering wave function, given by (cf. eq. $(3.10))$ :

$$
f_{j n l n}\left(q_{n}, r_{n A}\right) \sim f_{j n l n}^{\text {asym. }}=j_{l n}\left(q_{n} r_{n A}\right)+O_{j_{n} l_{n}} h_{L_{n}}^{(+)}\left(q_{n} r_{n A}\right),
$$

where the elastic partial wave scattering amplitude $O_{j n}$ in connected with the phase shift $\delta_{j n l n}$ and the s-matrix element $S_{j n} l_{n}$ of the elastic neutron target scattering by :

$$
O_{j n l n}=i e^{i \delta_{j u l n}} \sin \delta_{j u l n}=\frac{1}{2}\left(e^{2 i \delta_{j n l n}}-1\right)=\frac{1}{2}\left(S_{j n l n}-1\right) \text {. }
$$


In order to see how good the replacement (4.35) really is, we plot in figs. 3,4 the quantity :

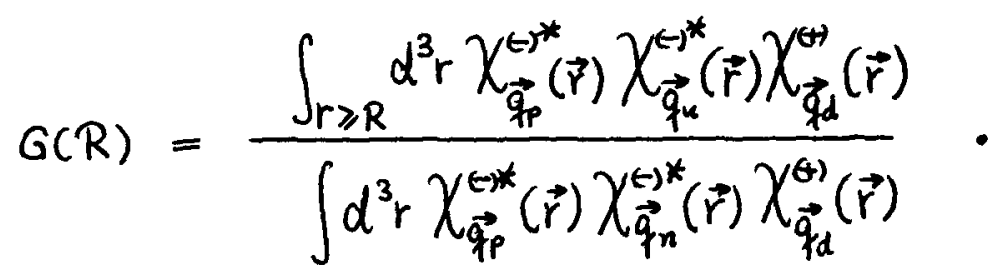

(For further details see ref. [82]). It can be seen that for lower energies (see fig. 3 for $E_{d}=12 \mathrm{MeV}$ ) contributions from the nuclear interior are completely negligible, while for higher energies, (see fig. 4 for $\mathrm{E}_{d}=18 \mathrm{MeV}$ ), a small but non-negligible contribution from the nuclear interior shows up. Nevertheless, even for energies well above the coulomb barrier ( $E_{d} \succsim 20 \mathrm{MeV}$ ) the contributions are quite small, due to the strong suppression by non-locality and finitorange effects.

For a more accurate treatment of the neutron wave function we approximate the post-form T-matrix element $(3.22)$ by

$$
T_{f i}^{(\theta)}=\int d^{3} r_{p A}{ }^{c} \chi_{\vec{q}_{p}}^{(-) *}\left(\vec{r}_{p A}\right) F_{L M}\left(\vec{r}_{p A}\right) c \chi_{\vec{q}_{\alpha}^{(t)}}^{\left(\vec{r}_{p A}\right)},
$$

with the "form factor":

$$
\begin{aligned}
& F_{L M}\left(\vec{r}_{p A}\right)=\int d^{3} r_{u p} X_{\vec{q}_{n}}^{\in *}\left(\vec{r}_{u A}\right) V_{n p}\left(\vec{r}_{n p}\right) u_{L}\left(r_{n p}\right) Y_{L M}\left(\hat{r}_{n p}\right) \\
& =4 \pi \sum_{\ln m_{n}} i^{-\ln } Y_{\ln m_{n}}^{*}\left(\hat{q}_{n}\right) . \\
& \quad \cdot \int d^{3} r_{n p} f_{j n l n}\left(q_{u}, r_{u A}\right) Y_{l_{n} m_{n}}\left(\hat{r}_{n A}\right) V_{u p}\left(\vec{r}_{u p}\right) u_{L}\left(r_{u p}\right) Y_{L M}\left(\hat{r}_{u p}\right) .
\end{aligned}
$$

The great simplification of the T-matrix (4.38) occurs since we introduced an approximation first proposed by Dar et al. $[84,85]$ for sub-Coulomb stripping to bound states. These authors claim that the small effects due to the "polarization" of the deuteron can be simulated by replacing the argument in the elastic scatte- 
ring deuteron wave function $c \chi_{\vec{q}_{d}}^{(t)}\left(\vec{r}_{d A}\right)$ by ${ }^{c} \chi_{\vec{q}_{d}^{(t)}}^{\left(\vec{r}_{p A}\right)}$. We will show later on whether this statement is correct or not and use it for the moment as a prescription. For our further calculation we use the general additon theorem of Sawaguri and Tobocman $[73]$ for an arbitrary function $h\left(\vec{r}_{1}+\vec{r}_{2}\right)$ :

$$
\begin{aligned}
& h\left(\vec{r}_{1}+\vec{r}_{2}\right)=\frac{4}{\sqrt{\pi}} \sum_{\substack{l l_{1} l_{2} \\
m}} i^{l_{1}+l_{2}-l} \hat{l}_{1} \hat{l}_{2} \hat{l}\left(\begin{array}{lll}
l_{1} & l_{2} & l \\
m_{1} & m_{2} & m
\end{array}\right)\left(\begin{array}{lll}
l_{1} & l_{2} & l \\
0 & 0 & 0
\end{array}\right) Y_{l_{1} m_{1}}\left(\hat{r}_{1}\right) Y_{l_{2} m_{2}}\left(\hat{r}_{2}\right) \text {. } \\
& \text { - } \int_{0}^{\infty} d k k^{2} j_{l_{1}}\left(k r_{1}\right) j_{l_{2}}\left(k r_{2}\right) \int d^{3} r j_{l}(k r) Y_{l m}(\hat{r}) h(\vec{r}),
\end{aligned}
$$

and obtain (cf. ref. $[86]$ )

$$
\begin{aligned}
F_{L M}\left(\vec{r}_{p A}\right)=4 \pi & \sum_{\substack{l_{2} m_{2} \\
l_{n} m_{n}}} i^{L+l_{2}-2 l_{n}} Y_{l_{n} m_{n}}^{*}\left(\hat{q}_{n}\right) Y_{L_{2} m_{2}}^{*}\left(\hat{r}_{p A}\right) \hat{L} \hat{l}_{2} \hat{l}_{n} \\
& \left(\begin{array}{lll}
L & l_{2} & l_{n} \\
M & m_{2} & m_{n}
\end{array}\right)\left(\begin{array}{ccc}
L & l_{2} & l_{n} \\
0 & 0 & 0
\end{array}\right) Z_{j n l_{n}}^{L l_{2}}\left(r_{p A}\right),
\end{aligned}
$$

where the radial form factor is given by *)

$$
Z_{j_{n} l_{n}}^{L l_{2}}\left(r_{p p}\right)=\frac{4}{\sqrt{\pi}} \int_{0}^{\infty} d k k^{2} j_{L_{2}}\left(k r_{p \beta}\right) A_{L}(k) \int_{0}^{\infty} d r r^{2} j_{l_{n}}(k r) f_{j_{n} l_{n}}\left(q_{n}, r\right),
$$

with

$$
A_{L}(k)=\int_{0}^{\infty} d r_{n p} r_{n p}^{2} j_{L}\left(k r_{n p}\right) V_{n p}\left(r_{u p}\right) u_{L}\left(r_{n p}\right)
$$

*) Some word of caution is necessary for the use of the general addition theorem (4.40). For "physical" wave functions, regular at the origin, this theorem can be well applied; however, for singular wave functions like $h_{l}^{(t)}\left(q_{n} r\right)$ it can not be used since the change of the integration order implied in eq. (4.42) is not allowed in this case. 
Using the Schrödinger equation for the bound deuteron we can write :

$$
A_{L}(k)=-\frac{\hbar^{2}}{2 m_{n p}}\left(k^{2}+\alpha^{2}\right) U_{L}(k)
$$

where the Fourier transform of the radial deuteron wave function is given by

$$
U_{L}(k)=\int_{0}^{\infty} d r r^{2} j_{L}(k r) u_{L}(r) .
$$

The radial form factor (4.42) can be more simplified by regarding only the s-state of the deuteron. Assuming a Hulthon wave function for the bound deuteron we immediately get :

$$
A_{0}(k)=\frac{D_{0}^{0}}{\sqrt{4 \pi}} \frac{\beta^{1 / 2}(\beta+\alpha)^{3 / 2}}{\beta^{2}+k^{2}},
$$

and for the radial form factor we obtain :

$$
\begin{aligned}
& Z_{j_{n} l_{n}}^{0 l_{n}}=\left(-\beta^{3}\right) A_{0}(0)\left\{h_{l_{n}}^{(+)}\left(i \beta r_{p R}\right) \int_{0}^{\rho^{a}} d x x^{2} f_{f_{n} \ln }\left(q_{u}, x\right) j_{l_{n}}(i \beta x)\right. \\
& \left.+j_{l_{n}}\left(i \beta r_{p A}\right) \int_{r_{p A}}^{\infty} d x x^{2} f_{j_{n} l_{n}}\left(q_{n}, x\right) h_{l_{n}}^{(t)}(i \beta x)\right\} \text {. }
\end{aligned}
$$

For the sake of simplicity we assume for the moment that the neutron-target interaction can be described by a complex square well as given e.g. by Feshbach et al. [87]:

$$
V_{n A}\left(r_{n A}\right)=-\theta\left(R_{n}-r_{n A}\right)\left(\bar{V}_{n A}+i \bar{W}_{n A}\right)
$$

with

$$
\bar{V}_{n A}=42 \mathrm{Mer}, \bar{W}_{n A}=0.03 \bar{V}_{n A} \text { and } R_{n}=1.45 \mathrm{~A}^{1 / 3} \text {. }
$$

In this case the neutron wave function for $r_{n A} \leqslant R_{n}$ is given by 


$$
f_{j n l_{n}}\left(q_{n}, r_{n \theta}\right)=K_{l_{n}} \cdot \dot{j}_{l_{n}}\left(K_{n} r_{n}\right),
$$

with

$$
x_{n}=\sqrt{\frac{2 \mu_{n}}{\hbar^{2}}\left(\bar{V}_{n A}+i \bar{W}_{n A}\right)}
$$

and where now the constant $K_{l_{n}}$ and the phase shifts in eq. (4.35) are determined by the matching condition at $r_{n A}=R_{n}$.

Using this simple wave function in eq. (4.42) gives

$$
\begin{aligned}
& Z_{j_{n} l_{n}}^{O l_{n}}\left(r_{p p}\right)=n_{0}\left(x_{n}\right) K_{l_{n}} j_{l_{n}}\left(x_{n} r_{p \theta}\right) \\
& +A_{0}\left(x_{n}\right) K_{l_{n}} R_{n}^{2} \beta \frac{q_{n}^{2}-K_{n}^{2}}{x_{n}^{2}+\beta^{2}} j_{l_{n}}\left(i \beta K_{p \theta}\right)\left(K_{n} k_{l n}^{(t)}\left(i \beta R_{n}\right) j_{l n}^{\prime}\left(K_{n} R_{n}\right)\right. \\
& -i \beta\left(\hat{h}_{l n}^{\prime \prime}\left(i \beta R_{n}\right) j_{l n}\left(x_{n} R_{n}\right)\right) \quad \text { for } r_{n A} \leqslant R_{n}
\end{aligned}
$$

and

$$
\begin{aligned}
& Z_{j n}^{O \ln }\left(r_{p \theta}\right)=A_{0}\left(q_{n}\right)\left(j_{l n}\left(q_{n} r_{p} \theta\right)+O_{j n} h_{n} h_{l n}^{(+)}\left(q_{n} r_{p \theta}\right)\right) \\
& +A_{0}\left(q_{n}\right) K_{l n} R_{n}^{2} \beta \frac{q_{n}^{2}-K_{n}^{2}}{K_{n}^{2}+\beta^{2}} h_{l n}^{(+)}\left(i \beta r_{p}\right)\left(K_{n} j_{l_{n}}\left(i \beta R_{n}\right) j_{l_{n}}^{\prime}\left(K_{n} R_{n}\right)\right. \\
& \left.-i \beta j_{l n}^{\prime}\left(i \beta R_{n}\right) j_{l n}\left(k_{n} R_{n}\right)\right) \text { for } r_{n A} \geqslant R_{n} \text {. }
\end{aligned}
$$

Neglecting the small correction terms in (4.5lb) and the interior part of the radial form factor (4.5la) we get the simple form factor already discussed in ref. [28]. In this ref. we used the asymptotic wave functions from the beginning and we obtained with the help of the well-known addition theorem for the Hankel function $[88]$ :

$$
F_{00}\left(\vec{r}_{p A}\right) \sim 4 \pi A_{0}\left(q_{n}\right) \sum_{\ln m_{n}} i^{-\ln } Y_{l n}^{*} m_{n}\left(\hat{q}_{n}\right) Y_{l n m_{n}}\left(\hat{r}_{p A}\right) f_{j_{n} l_{n}}^{\text {asym. }}\left(q_{n}, r_{p A}\right) \text {. }
$$


In fig. 5 we have plotted the approximate radial form factor used in eq. (4.52) and the exact radial form factor (4.51). It can be seen that the approximate form factor agrees very well with the exact one for $r_{p A} \gtrsim R_{n}$. According to our discussion above only this part contributes to the $\mathrm{T}$-matrix element in the sub-Coulomb region. Combining now (4.52) with eq. (4.38) we obtain $[28]$ :

$$
T_{f i}^{(t)}=(4 \pi)^{3 / 2} A_{0}\left(q_{n}\right) \sum_{l_{n} m_{n}} i^{-\ln } Y_{l_{n} m_{n}}\left(\hat{q}_{n}\right)\left(M_{l_{n} m_{n}}^{0}+O_{j_{n} l_{n}} M_{l_{n} m_{n}}^{+}\right),
$$

where

$$
M_{l_{n} m_{n}}^{0}=\int d^{3} r{ }^{c} \chi_{\vec{q}_{p}}^{(-) *}(\vec{r}) j_{l_{n}}\left(q_{n} r\right) Y_{l_{n} m_{n}}^{*}(\hat{r}){ }^{c} \chi_{\vec{q}_{d}}^{(t)}(\vec{r})
$$

and

$$
M_{l_{n} m_{n}}^{+}=\int d^{3} r^{c} X_{\vec{q}_{p}}^{(\rightarrow *}(\vec{r}) h_{l_{n}}^{(+)}\left(q_{n} r\right) Y_{l_{n} m_{n}}^{*}(\hat{r}){ }^{c} X_{\vec{q}_{d}}^{(t)}(\vec{r}) .
$$

Expression (4.53) can be rewritten by splitting up again the plane wave part of the neutron scattering wave function as

$$
T_{f i}^{(+)}=(4 \pi)^{3 / 2} A_{0}\left(q_{n}\right)\left\{M_{p c}+\sum_{l_{n}} O_{j_{n} l_{n}} T_{l_{n}}^{n}\right\},
$$

with

$$
T_{l_{n}}^{n}=i^{-l_{n}} \sum_{m_{n}} Y_{l_{n} m_{n}}\left(\hat{q}_{n}\right) M_{l_{n} m_{n}}^{+}
$$

This is the extremely simple expression describing the subCoulomb deuteron break-up. With the help of a Hulthen wave function for the bound deuteron we get, according to eq. (4.46): 


$$
A_{0}\left(q_{n}\right)=\frac{D_{0}^{0}}{\sqrt{4 \bar{k}}} \frac{\sqrt{\beta}(\beta+\alpha)^{3 / 2}}{\beta^{2}+q_{n}^{2}}=\frac{A_{0}(0)}{1+\left(\frac{q_{n}}{\beta}\right)^{2}},
$$

where

$$
A_{0}(0)=\frac{D_{0}^{0}}{\sqrt{4 \pi}}\left(1+\frac{\alpha}{\beta}\right)^{3 / 2}=1.244 \frac{D_{0}^{0}}{\sqrt{4 \pi}} \simeq \frac{D_{0}}{\sqrt{4 \pi}} .
$$

For low neutron energies where $q_{n}$ is small, we have $A_{0}\left(q_{n}\right) \simeq A_{0}(0)$ and therefore the modification due to the finite-range of the neutron-proton interaction is quite small. This gives us further confidence in the zero-range approximation used betore.

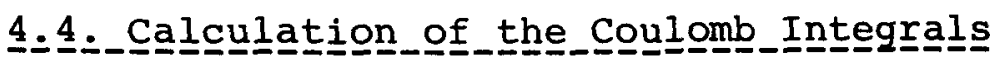

Using eq. (4.56) the numerical treatment of the sub-Coulomb break-up reaction reduces to the calculation of the three-dimensional integral eq. (4.55). The pure Coulomb matrix element

Mpcis, using eqs. $(4.17-4.20)$, easily obtainable. Such a closed analytical expression for $M_{\ln m_{n}}^{+}$is only available if $l_{n}=m_{n}=0$. In this case we have $[89,28]$ :

$$
\begin{aligned}
M_{00}^{+}= & \frac{\sqrt{4 \pi} e^{-\frac{\pi}{2}\left(\eta_{d}+\eta_{p}\right)}}{i q_{n}\left(\left(q_{d}-q_{p}\right)^{2}-q_{p}^{2}\right)} \Gamma\left(1+i \eta_{d}\right) \Gamma\left(1+i \eta_{p}\right)\left(\frac{q_{p}^{2}-\left(q_{d}+q_{n}\right)^{2}}{\left(\vec{q}_{d}-\vec{q}_{p}\right)^{2}-q_{n}^{2}}\right)^{i \eta_{d}} \\
& \cdot\left(\frac{q_{d}{ }^{2}-\left(q_{p}+q_{n}\right)^{2}}{\left(\overrightarrow{q_{d}}-\vec{q}_{p}\right)^{2}-q_{n}^{2}}\right)^{i \eta_{p}} \frac{{ }_{2} F_{1}\left(-i \eta_{d}-i \eta_{p} ; 1_{j}-\tilde{\xi}\right)}{1+\tilde{\xi}},
\end{aligned}
$$

with

$$
\tilde{\xi}=\frac{4 q_{d} q_{p}}{\left(q_{\alpha}-q_{p}\right)^{2}-q_{n}^{2}} \sin ^{2} \frac{\theta_{p}}{2} .
$$


The use of this simple expression not only reduces the computing time further but also gives us a simple description of the behaviour of the break-up reaction at low neutron energles. Using the well-known low energy behaviour of the phase shifts (see e.g. ref. $[90$, vol.I, pg.392]) :

$$
e^{i \delta_{l_{n}}} \sin \delta_{l_{n}} \simeq \sin \delta_{l_{n}} \simeq \delta_{l_{n}} \rightarrow \frac{l_{n}+1-\hat{q}_{l_{n}}}{l_{n}+\hat{q}_{l_{n}}} \frac{\left(q_{n} R_{n}\right)^{2 l_{n}+1}}{\left(2 l_{n}-1\right) ! !\left(2 l_{n}+1\right) ! !},
$$

where $\hat{q}_{l_{n}}$ denotes the quantity

$$
\hat{q}_{l_{n}}=\left.\lim _{q_{n} \rightarrow 0} \frac{d\left(r f_{j k l n}\right) / d r}{f_{j n l n}}\right|_{r=R_{n}}
$$

and the expansion $[77]$

$$
h_{l n}^{(+)}\left(q_{n} r\right) \simeq i n_{l_{n}}\left(q_{n} r\right) \simeq-i \frac{\left(2 l_{n}-1\right) ! !}{\left(q_{n} r\right)^{l_{n}+1}} \quad \text { for } q_{n} r \ll 1
$$

we see that only the s-wave contributes in the low energy limit.

Therefore we have

$$
T_{f i}^{(+)} \simeq 4 \pi D_{0}\left\{M_{p c}+\frac{O_{0}}{\sqrt{4 \pi}} M_{00}^{+}\right\}
$$

Furthermore, with $[77]$

$$
j_{l_{n}}\left(q_{n} r\right) \simeq \frac{\left(q_{n} r\right)^{l_{n}}}{\left(2 l_{n}+1\right) ! !} \quad \text { for } \quad q_{n} r \ll 1
$$

we conclude that for $q_{n} r \ll 1$

$$
M_{p C} \simeq \frac{1}{\sqrt{4 \pi}} M_{00}^{0}
$$


$-47-$

From eg. (4.60) one derives the general relation

$$
M_{00}^{\circ}=e^{i \frac{\gamma-\bar{k}}{2}} \sin \frac{\gamma}{2} M_{00}^{+}
$$

where

$$
\gamma=\eta_{d} \log \frac{\left(q_{d}+q_{n}\right)^{2}-q_{p}^{2}}{\left(q_{d}-q_{n}\right)^{2}-q_{p}^{2}}+\eta_{p} \log \frac{q_{d}^{2}-\left(q_{p}+q_{n}\right)^{2}}{q_{d}^{2}-\left(q_{p}-q_{n}\right)^{2}}
$$

In the limit of $q_{n} \rightarrow 0$ we therefore finally obtain :

$$
T_{f i}^{(t)}=\sqrt{4 \pi} D_{0} \Pi_{00}^{0}\left(q_{i}=0\right)\left\{1+\frac{a_{0}}{2} \frac{q_{d}^{2}-q_{p}^{2}}{\eta_{d} q_{d}-\eta_{p} q_{p}}\right\},
$$

where $a_{0}$ is the usual scattering length *).

If, in general, phase shifts with $l_{n}>0$ contribute to the T-matrix element, it is not possible to obtain such an easy closed expression as eq. (4.60). Therefore, we perform a partial wave expansion in the incident beam coordinate system, leading to

$$
\begin{aligned}
M_{l_{n} m_{n}}^{+}=(4 \pi) \hat{l}_{n} \sum_{l_{d} l_{p}} & i^{l_{d}-l_{p}} e^{i\left(\sigma_{d}\left(\eta_{d}\right)+\sigma_{l_{p}}\left(\eta_{p}\right)\right)} \hat{l}_{d} \hat{l}_{p}^{2} Y_{l_{p}, m_{n}}\left(\theta_{p}, 0\right) \\
& \left(\begin{array}{ccc}
l_{d} & l_{n} l_{p} \\
0 & 0 & 0
\end{array}\right)\left(\begin{array}{ll}
l_{d} & l_{n} l_{p} \\
0 & m_{n}-m_{n}
\end{array}\right) R_{l_{d} l_{n} l_{p}}^{+},
\end{aligned}
$$

with

$$
R_{l_{d} l_{n} l_{p}}^{+}=\frac{1}{q_{d} q_{p}} \int_{0}^{\infty} d r F_{l d}\left(\eta_{d}, q_{d} r\right) h_{l_{n}}^{(t)}\left(q_{n} r\right) F_{l_{p}}\left(\eta_{p}, q_{p} r\right) .
$$

A similar expansion of $M_{l_{n} m_{n}}^{0}$ leads to the same expression excopt that $R_{l_{d} l_{n} l_{p}}^{+}$is to be replaced by $R_{l_{d} \ln l_{p}}^{0}$, defined in eq. (4.16) and where

$$
R_{l_{d} \ln l_{p}}^{0}=\operatorname{Re}\left(R_{l_{d} l_{n} l_{p}}^{+}\right) \text {. }
$$

*) This result can be compared with the results of the WatsonMigdal theory $[91,92]$. 
While, in principle, the calculation of the integrals eq. (4.72) can be performed by Vincent and Fortune's integration technique, an introduction of the methods used in the theory of coulomb excitation $[74]$ leads to a rather drastic reduction of computing time. In this theory it has been shown that such integrals can be calculated exactly by means of generalized hypergeometric functions with two variables, so-called Appell functions $[93]$. Writing $[77]$

$$
h_{l_{n}}^{(t)}(z r)=i^{-\ln _{n}-1} \frac{e^{i z r}}{z r} \sum_{n=0}^{\ln } \frac{\left(l_{n}+n\right) !}{n !\left(l_{n}-n\right) !}(-2 i z r)^{-n},
$$

we get

$$
R_{l_{d} \ln l p}^{+}=\lim _{x \rightarrow 0+}\left\{\frac{i^{-\ln -1}}{z} \sum_{n=0}^{l_{n}} \frac{(\ln +n) !}{n !(\ln -n) !}(-2 i z)^{-n} M_{L_{d, l p}}^{-n-1, i z}\right\},
$$

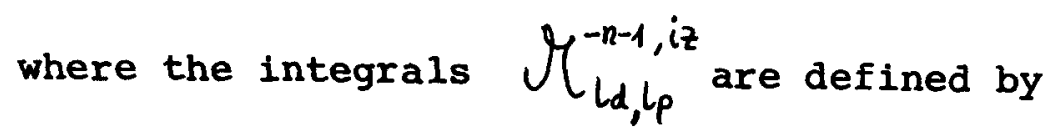

$$
\mathscr{K}_{l_{d, l p}}^{-n-1, i z}=\frac{1}{q_{d} q_{p}} \int_{0}^{\infty} d r F_{l_{\alpha}}\left(\eta_{\alpha}, q_{d} r\right) \frac{e^{i z r}}{r^{n+1}} F_{L_{p}}\left(\eta_{p}, q_{p} r\right),
$$

and where

$$
z=q_{n}+i k \quad \text { with } k>0 \text {, }
$$

was introduced to make the integral (4.76) well behaved for further analysis. It is easy to see that no numerical complication arises from taking the limit $x \rightarrow 0$, which is in striking contrast to the direct numerical integration of (4.72) after having introduced such a convergence factor $[38]$. 
The integrads (4.76) are quite similar to the integrals occurring in the coulomb excitation and sub-Coulomb transfer reactions to bound states $[94]$. While the first ones are obtained by setting $z=0$, the latter are given by $q_{n}=0$. Using the results of refs. $[74,94,32]$ we can write

$$
\begin{aligned}
\mathscr{I}_{l_{d, l}, l_{p}}^{-n-i z}= & e^{-\frac{\pi}{2}\left(\eta_{d}+\eta_{p}\right)} \frac{\left|\Gamma\left(l_{d}+1+i \eta_{d}\right) \| \Gamma\left(l_{p}+1+i \eta_{p}\right)\right|}{\left(2 l_{d}+1\right) !\left(2 l_{p}+1\right) !}\left(l_{d}+l_{p}+1-n\right) ! \\
& \cdot\left(q_{d}-q_{p}+z\right)^{n-2}(x)^{l_{d}}(-y)^{l_{p}} i^{l_{d}+l_{p}-n+2} \\
& \cdot F_{2}\left(l_{d}+l_{p}-n+2, l_{d}+1+i \eta_{\alpha}, l_{p}+1-i \eta_{p} ; 2 l_{d}+2,2 l_{p}+2 ; x, y\right),
\end{aligned}
$$

with

$$
x=\frac{2 q_{d}}{q_{d}-q_{p}+z}, \quad y=\frac{-2 q_{p}}{q_{d}-q_{p}+z} \text {. }
$$

The $F_{2}$-function can for $|x|+|y|<1$ be calculated by its power series :

$$
F_{2}\left(\alpha, \beta, \beta^{\prime} ; \gamma, \gamma^{\prime} ; x, y\right)=\sum_{m n} \frac{(\alpha)_{m+n}(\beta)_{m}\left(\beta^{\prime}\right)_{n}}{(\gamma)_{m}\left(\gamma^{\prime}\right)_{n} m ! n !} x^{m} y^{n}
$$

Since, due to the kinematical condition (3.26), this power series does not converge, we must use a suitable analytic continuation. This can be done in an especially simple way if $h_{d}=l_{p} \pm n$ (so-called "maximal matrix elements"). In this case we get (for further details see refs. $[74,94,32]$ ): 


$$
\begin{aligned}
& \mu_{l_{p+n, l_{p}}^{-n-1, i z}}=\frac{\left|\Gamma\left(l_{p}+1+i \eta_{p}\right)\right|}{\left|\Gamma\left(l_{p}+n+1+i \eta_{\alpha}\right)\right|}\left(\frac{q_{p}}{q_{d}}\right)^{l_{p}}\left(2 q_{d}\right)^{n-2} e^{\frac{\pi}{2} \xi} . \\
& \text { - }\left\{\frac{|\Gamma(n+i \xi)|^{2}}{(2 n-1) !} F_{2}\left(-2 n+1, l_{p}+1-i \eta_{p}, l_{p}+1+i \eta_{p}-n+1-i \xi,-n+1+i \xi ; u, v\right)\right. \\
& +\frac{\Gamma(-n-i \xi) \Gamma\left(l_{p}+n+1-i \eta_{d}\right)}{\Gamma\left(l_{p}+1-i \eta_{p}\right)}\left(e^{i \pi} u\right)^{i \xi+n} \text {. } \\
& \text { - } F_{2}\left(-n+1+i \xi, l_{p}+n+1-i \eta_{d}, l_{p}+1+i \eta_{p} ; n+1+i \xi,-n+1+i \xi ; u, v\right) \\
& +\frac{\Gamma(-n+i \xi) \Gamma\left(l_{p}+n+1+i \eta_{\alpha}\right)}{\Gamma\left(l_{p}+1+i \eta_{p}\right)}\left(e^{-i \pi} v\right)^{-i \xi+n} \text {. } \\
& \text { - } \left.F_{2}\left(-n+1-i \xi, l_{p}+n+1+i \eta_{d}, l_{p}+1-i \eta_{p} ; n+1-i \xi,-n+1-i \xi ; v, u\right)\right\} \text {, }
\end{aligned}
$$

with

$$
u=\frac{q_{d}-q_{p}-z}{2 q_{d}}, \quad v=\frac{q_{d}-q_{p}+z}{2 q_{d}},
$$

and

$$
\xi=\eta_{p}-\eta_{\alpha}
$$

Expression (4.81) is well suited for a numerical treatment since the first $F_{2}$ - function is only a polynomial and the other $F_{2}$-functions can easily be computed from their series expansion (4.80) . The other "maximal matrix elements" $\varkappa_{l_{p}-n, l_{p}}^{-n-1, i z}$ can be obtained from eq. (4.81) by the general symmetry relation :

$$
M_{L_{d, l p}}^{-n-1, i z}\left(\eta_{d}, \eta_{p} ; q_{d}, q_{p}\right)=M_{L_{p, l d}}^{-n-1, i z}\left(\eta_{p}, \eta_{d} ; q_{p}, q_{d}\right) .
$$

In some special cases other analytic continuations may wo ik even better, e.g. using olson's Fp-function $[95,96]$, as was shown in ref. $[32]$. 
If $n=0\left(l_{d}=l_{p}=l\right)$ eq. $(4.81)$ can be considerably reduced further, leading to the important special case $[94,32]$ :

$$
\begin{aligned}
K_{l, l}^{-1, i z}= & \frac{\left|\Gamma\left(l+1+i \eta_{d}\right) \| \Gamma\left(l+1+i \eta_{p}\right)\right|}{(2 l+1) !}\left(x_{0}\right)^{l} \cdot\left(\frac{q_{d}-q_{p}-z}{q_{d}+q_{p}-z}\right)^{i \eta_{p}}\left(\frac{q_{d}+q_{p}+z}{q_{d}-q_{p}-z}\right)^{i \eta_{d}} \\
& \frac{e^{-\frac{\pi}{2} \xi}}{\left(q_{d}+q_{p}\right)^{2}-z^{2}}{ }_{2} F_{1}\left(l+1-i \eta_{d}, l+1+i \eta_{p} ; 2 l+2 ; x_{0}\right),
\end{aligned}
$$

where

$$
x_{0}=\frac{4 q_{d} q_{p}}{\left(q_{d}-q_{p}\right)^{2}-z^{2}}
$$

In ref. $[32]$ we have shown in detail how the "non maximal matrix elements" necessary for the evaluation of $R_{l_{d} \ln l_{p}}^{+}$using eq. (4.75) can be calculated with the help of recursion relations. All these recursion relations are contained in the general relation

$$
\begin{aligned}
& x_{1} C_{l_{p}+1} Z_{l_{d}, l_{p+1}}+x_{2} C_{l_{d}} Z_{l_{d}-1, l_{p}}-x_{3} C_{l_{d}+1} Z_{l_{d+1}, l_{p}}-x_{4} C_{l_{p}} Z_{l_{d}, l_{p}-1} \\
& -\left(x_{1} \frac{\eta_{p} q_{p}}{l_{p}+1}+x_{2} \frac{\eta_{d} q_{d}}{l_{d}}-x_{3} \frac{\eta_{d} q_{d}}{l_{d}+1}-x_{4} \frac{\eta_{p} q_{p}}{l_{p}}\right) Z_{l_{d}, l_{p}} \\
& =\left(x_{1}+x_{4}\right) \int_{0}^{\infty} d r u_{l_{d}}\left(\eta_{d}, q_{d} r\right) \frac{d h(r)}{d r} u_{l_{p}}\left(\eta_{p}, q_{p} r\right) \\
& +\left(x_{1}\left(l_{p}+1\right)+x_{2} l_{d}-x_{3}\left(l_{d}+1\right)-x_{4} l_{p}\right) \int_{0}^{\infty} d r u_{l_{d}}\left(\eta_{d}, q_{d} r\right) \frac{\left.l_{(r}\right)}{r} u_{l_{p}}\left(\eta_{p}, q_{p} r\right),
\end{aligned}
$$

with

$$
Z_{L_{d, l p}}=\int_{0}^{\infty} d r u_{L_{d}}\left(\eta_{d}, q_{d} r\right) h(r) u_{L_{p}}\left(\eta_{p}, q_{p} r\right),
$$

where $u_{\iota}(\eta, q r)$ is the regular or irregular coulomb function or a linear combination of them and $h(r)$ is any analytic function so that the integral $(4.88)$ is convergent. 


$$
C_{l_{\nu}}=q_{\nu} \frac{\left|l_{\nu}+i \eta_{\nu}\right|}{l_{\nu}} \text { with } \quad \nu=d_{1} p
$$

The $x_{i}$ are arbitrary quantities, satisfying the condition

$$
x_{1}+x_{2}+x_{3}+x_{4}=0 \text {. }
$$

By giving appropriate values to these numbers we can eliminate from (4.87) the unwanted matrix elements and derive numerous recursion relations. So we can construct relations for the "maximal matrix elements" and other ones which allow the calculation of the "non-maximal matrix elements" from the "maximal matrix elements". By using these recursion relations in the backward direction with asymptotic starting values the numerical procedure is very stable. Since furthermore only four (!) maximal matrix elements have to be computed directly in order to normalize the matrix elements obtained from the recursion relations, this method allows a very quick computation of all the radial integrals necessary to calculate $M_{\ln m_{n}}^{+}$. We do not want to go into further details of this procedure but refer the reader to ref. $[32]$.

Finally, we mention the semiquantal approximation of the $\mathrm{T}$ matrix eq. (4.56). This approximation consists of replacing only the radial integrals in eq. (4.71) by their semiclassical limit.

Using the well-known WKB approximations for the Coulomb functions and neglecting rapidly oscillating terms we get : 


$$
R_{l_{d} \ln l_{p}}^{+} \simeq \frac{1}{2 q_{d} q_{n} q_{p}} I_{l_{n} \mu}^{+}\left(\varepsilon, \xi, \xi^{\prime}, \rho\right),
$$

with

$$
\begin{gathered}
I_{l_{n} \mu}^{+}\left(\varepsilon, \xi, \xi^{\prime}, \rho\right)=\frac{1}{2} \int_{-\infty}^{+\infty} d w e^{i\left(\xi^{\prime} \varepsilon \sinh \omega+\xi \omega\right)} \rho(1+\varepsilon \cosh \omega) \\
\cdot h_{l_{n}}^{(+)}(\rho(1+\varepsilon \cosh \omega))\left(\frac{\cosh \omega+\varepsilon+i \sqrt{\varepsilon^{2}-1} \sinh \omega}{1+\varepsilon \cosh \omega}\right)^{\mu} .
\end{gathered}
$$

Here, we have introduced the quantities

$$
\begin{array}{ll}
\eta=\frac{1}{2}\left(\eta_{d}+\eta_{p}\right), & \rho=q_{n} a_{c}, a_{c}=\frac{\eta}{Q}, Q=\frac{1}{2}\left(q_{d}+q_{p}\right), \\
\xi^{\prime}=a_{c}\left(q_{d}-q_{p}\right), & \mu=l_{p}-l_{d}, \\
\varepsilon=\sqrt{1+\frac{l(l+1)}{\eta^{2}},}, l=\frac{1}{2}\left(l_{d}+l_{p}\right) .
\end{array}
$$

The integral $I_{\ln \mu}^{+}$converges because of the rapid oscillations at infinity. From the numerical point of view it is useful to change the integration variable $w$ by $w+i \frac{\pi}{2}$. Since the integrand has no poles between the two contours we obtain

$$
\begin{gathered}
I_{\ln \mu}^{+}\left(\varepsilon, \xi, \xi^{\prime}, \rho\right)=\frac{1}{2} e^{-\frac{\pi}{2} \xi} \int_{-\infty}^{+\infty} d w e^{-\xi^{\prime} \varepsilon \cosh \omega+i \xi \omega} \rho(1+i \varepsilon \sin h \omega) \cdot \\
\cdot h_{l n}^{(+)}(\rho(1+i \varepsilon \sinh \omega))\left(\frac{i \sinh \omega+\varepsilon-\sqrt{\varepsilon^{2}-1} \cosh \omega}{1+i \varepsilon \sinh \omega}\right)^{\mu} .
\end{gathered}
$$

The integrand now behaves at infinity like exp $\left(-\frac{1}{2}\left(\xi^{\prime}-\rho\right) \varepsilon e^{\omega}\right)$. From the kinematical condition (3.26) it follows that $\xi^{\prime}>\rho$ from which it becomes obvious that the integral (4.94) converges rapidly. The inequality $\xi^{\prime}>\rho$ holds for any reaction where the projectile mass is smaller than the target mass, as is usually the case. For $\xi^{\prime}<\rho$, which occurs in the opposite case, the contour of the integration has to be suitably deformed. The 
divergent case $\xi^{\prime}=\rho$ cannot occur for kinematical reasons.

In fig. 6 we have shown the accuracy of the semiclassical approximation of the radial integral for a special example. From this it can be seen that this method can also be well used for a fast and rather accurate computation of the T-matrix element.

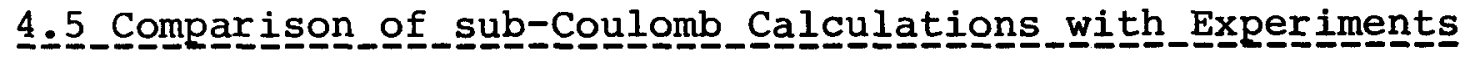

After having described in detail how to calculate the T-matrix in the sub-Coulomb energy region we are now able to compare calculated theoretical differential cross sections, using the definition (2.16), with experiments. For this purpose we have chosen very accurate coincidence measurements by Lang et al. By calculating the theoretical cross section only the phase shifts of the elastic neutron-target scattering have to be known. It is very important that on $\perp y$ these model independent parameters enter into the sub-Coulomb T-matrix.

In this context it is interesting to compare this result with the plane-wave theory of continuum stripping reactions given by Lipperheide $[50]$. He found that the cross section depends on the off-shell scattering amplitude of the neutron-target scattering. Therefore, the strong coulomb distortion brings, so to speak, the scattering amplitude completely back to the energy shell.

Here, we generate the phase shifts by an optical potential of 
the Woods-Saxon form, eqs. $(4.32,4.33)$, given by Becchetti and Greenlees $[97]$. This potential describes very well the elastic scattering of neutrons on heavy nuclei. In addition, our calculations show that the potential of Villiers et al. [98] which is of the Gaussian form gives also nearly the same results.

As a first example we show the energy dependence of the breakup reaction on gold. In fig. 7 the energy-integrated coincidence cross section is compared with our theoretical results showing a very good agreement. Two sets of neutron and proton angles have been chosen, $\theta_{n} \equiv 40^{\circ}, \theta_{p}=60^{\circ}$ and $\theta_{n}=40^{\circ}, \theta_{p}=80^{\circ}$ with a coplanar geometry $\left(\Phi=0^{\circ}\right)$. The predictions of the pure Coulomb theory of Landau and Lifshitz are also shown to emphasize the importance of the neutron-target interaction. Indeed, even at very low energies this interaction can never be neglected, because it reduces the pure luulomb cross section by about $25 \%-$ 508. This strong influence of the nuclear interaction is in agreement with the results of $\operatorname{refs} \cdot[25,27]$. In fig. 7 it can further be seen that at higher energies the nuclear deuteronnucleus interaction shows up and can, around the coulomb barrier, be described in a simple way by the diffraction model approach.

To show in more detail that our sub-Coulomb theory is able to explain the data quantitatively, we have compared in fig. 8 the theoretical angular dependence of the break-up cross section at $E_{d}=10.5 \mathrm{MeV}$ on $\mathrm{Au}$ with experiments. Again we see that the pure coulomb theory gives a rough estimate of the cross section. Furthermore, the small influence of the deuteron-target interaction taken into account by the diffraction model approach 
can be seen especially at backward proton angles, where the deuteron comes nearest to the target.

Finally, in figs. 9 and 10 the excellent agreement between experiment and theory is shown for two coincidence spectra at different neutron and proton angles for $\mathrm{E}_{\mathrm{d}}=12 \mathrm{MeV}$ on $\mathrm{Au}$. Of course, a triple differential cross section is the most crucial test for the T-matrix itself, so that we checked the high accuracy of the DWBA predictions. This may be the most important reason to study the sub-Coulomb deuteron break-up, since no free parameters enter into our expression. Therefore, we are able to test both the relative accuracy as well as the absolute accuracy of the DWBA. This is in contrast to the usual DWBA formalism for stripping reaction to bound states, where the so-called spectroscopic factor is adjusted by comparing the experiment with theory, making an absolute test of the DWBA impossible. Keeping in mind that the experimental results may have an absolute error of about $10 \% 15 \%$ [26] and that the theoretical calculation may also have an error of about 58 due to some small corrections (see below), and to the uncertainty of the neutrontarget interaction we have at least the result that the DWBAamplitude for deuteron induced reactions is correct within about 15\% $20 \%$. This gives further confidence in the extraction of spctroscopic factors in stripping reactions to bound states. More accurate measurements at even lower deuteron energies will lead to a further clarification of these points. 
On the other hand, taking the accuracy of the DWBA as manifested we can extract from sub-Coulomb break-up reactions some new information on the neutron-target phase shifts. Indeed, as was also shown in ref. $[25]$, this neutron-target interaction has a strong influence on the differential cross section. In fig. Il this dependence of the cross section on the optical model parameters for the neutron-target interaction is shown. Hereby, we have used the complex square well of Feshbach, Porter and Weisskopf for simplicity's sake. As another illustration of this dependence we show in fig. 12 the energy-integrated break-up cross section on ${ }^{197} \mathrm{Au}$ and ${ }^{208} \mathrm{~Pb}$. Using the optical potential parameters of Becchetti and Greenlees [97] the small A-dependence there leads to differences of up to $20 \%$ in the cross section, while the $\mathrm{z}$-dependence is negligible. This effect does not explain the characteristic strong variation of the break-up cross section for different target nuclei observed for the first time by Nemets et al. [22], but using a potential which takes specific shell effects into account will naturally give rise to such larger differences.

\section{Small Corrections}

As we have seen above, the sub-Coulomb break-up provides us with a clean test of the DWBA. To make such a test more effective all the small corrections which we have neglected in deriving eq. (4.56) should be carefully investigated. 


\section{1_Spin_or}

One approximation which we used in this context was the neglect of a spin-orbit force in the neutron-target interaction, which meant that the quantity $O_{\ell_{n} j_{n}}$ (see eq. (4.36)) became independent of $j_{n}$. Of course, it is not difficult to give up this approximation. We have recalculated the differential cross sections shown above by adding to the potential (4.32) a spin orbit term of the form

$$
V_{\text {so }}(r)=\bar{V}_{\text {so }} \frac{1}{r} \frac{d f\left(x_{s 0}\right)}{d x_{s 0}} \vec{\imath} \cdot \vec{s}
$$

The results, which we do not show explicitly, are practically the same as those obtained before. While at large neutron energies some small influence of $V_{\text {so }}(r)$ shows up, this effect can hardly be observed experimentally because the cross section in this energy region is very small.

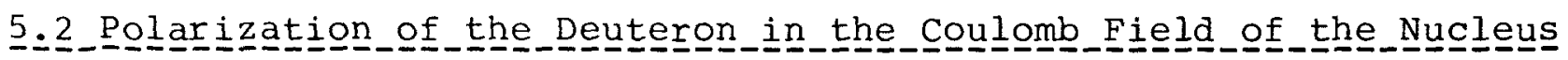

In the DWBA it is assumed that the internal wave function of the deuteron remains unchanged along its path. But, of course, when a deuteron comes close to the nucleus, the electric field of that nucleus will polarize the deuteron, pushing the proton away from the nucleus. This causes $T=1$ admixtures in the internal deuteron wave function. Estimates of the magnitude of these admixtures were given by Griffy $[99]$ and Drachmann $[100]$ using an adiabatic approximation. A more careful investigation in the case of sub-Coulomb stripping to bound states by Gibson and Kerman [5] gave much less dramatic effects than what was found in ref. [99]. 
Dar, de Shalit and Reiner claimed in their treatment of subCoulomb deuteron stripping that these "polarization" effects

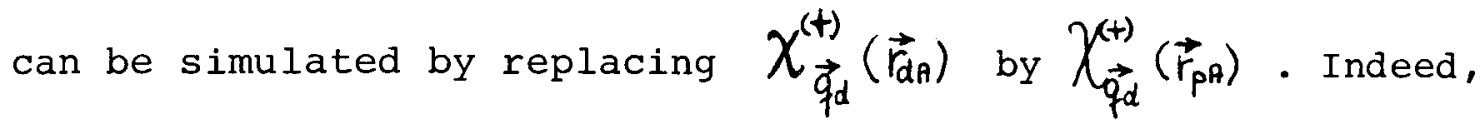
this "ansatz" describes a deuteron where the proton has a larger probability of staying away from the nucleus than the neutron. In order to investigate how well this "ansatz" takes the polarization into account, we write for the full wave function

$$
\Psi_{i}^{(+)}\left(\vec{r}_{d \theta}, \vec{r}_{n p}\right)=\chi_{\vec{q}_{d}}^{(+)}\left(\vec{r}_{d \theta}\right)\left(\Phi_{d}\left(\vec{r}_{n p}\right)+\tilde{\Phi}_{d}\left(r_{d A}, r_{n p}\right)\right)
$$

According to refs. $[5,101,102,84] \tilde{\Phi}_{d}$ is given in first order perturbation theory by

$$
\tilde{\Phi}_{d}\left(\vec{r}_{d A}, \vec{r}_{n p}\right) \simeq \frac{Z e^{2}}{r_{d A}^{2}} \frac{2 m_{n p}}{\hbar^{2}} \frac{N_{H}}{\sqrt{4 \pi}} g_{d}\left(r_{n p}\right) \cos \theta_{p},
$$

with

$g_{d}\left(r_{n p}\right)=\frac{e^{-\alpha r_{n p}}}{r_{n p}^{2}}\left\{\left(\frac{r_{n p}^{3}}{4 \alpha}-\frac{2\left(1+\alpha r_{n p}\right)}{\mu_{0}^{2}\left(2 \alpha+\mu_{0}\right)^{2}}\right)-\frac{e^{-\mu_{0} r_{n p}}}{\mu_{0}^{2}\left(2 \alpha+\mu_{0}\right)^{2}}\left(2+2 \beta r_{n p}+\mu_{0}(\beta+\alpha) r_{n p}^{2}\right)\right\}(5.4)$

where we have neglected the slight influence of the D-state of the deuteron.

Restricting ourselves to the case of the pure coulomb break-up the T-matrix can now be written as

$$
T_{f i}^{(+)}=T_{p c}+T_{p o l},
$$


with

$T_{p o l}=\left\langle\chi_{\vec{q}_{p}}^{c}\left(\vec{r}_{p A}\right) e^{i \vec{q}_{n} \vec{r}_{n} A}\left|V_{n p}\left(\vec{r}_{n p}\right)\right|^{c} \chi_{\vec{q}_{d}}^{(+)}\left(\vec{r}_{d A}\right) \tilde{\Phi}_{d}\left(\vec{r}_{d A}, \vec{r}_{r_{p}}\right)\right\rangle$.

Instead of solving this six-dimensional integral exactly we use the so-called "local WKB approximation" or the "local energy approximation" (LEA) which has been shown $[4,103-106]$ to be a highly successful approximate procedure for the finite range integration in bound state stripping reactions. Thus, we write

$$
\begin{gathered}
c \chi_{\vec{q}_{p}}^{(-)}\left(\vec{r}_{p A}\right)=c \chi_{\vec{q}_{p}}^{(-)}\left(\vec{r}_{d A}-\frac{\vec{r}_{p p}}{2}\right)=e^{-\frac{\vec{r}_{n p}}{2} \vec{\nabla}_{p} c \chi_{\vec{q}_{p}}^{(-)}\left(\vec{r}_{d A}\right)} \\
\simeq e^{-\frac{i}{2} \vec{r}_{p p} \vec{q}_{p r} c} \chi_{\vec{q}_{p}}^{(-)}\left(\vec{r}_{d A}\right)
\end{gathered}
$$

where we have replaced the momentum operator $\vec{\nabla}_{p}$, differentiating with respect to $\vec{r}_{d A}$ in $c \chi_{\vec{q}_{p}}^{(-)}\left(\vec{r}_{d A}\right)$, by the local momentum of the wave function at the point $r_{d A}$ :

$$
q_{p r}\left(r_{d A}\right)=\left(\frac{2 \mu_{P}}{\hbar^{2}}\left(E_{p}-V_{p A}\left(r_{\alpha A}\right)\right)\right)^{1 / 2}
$$

$$
\begin{aligned}
& \text { Assuming that }[104] \\
& \qquad \begin{array}{l}
\vec{q}_{p r} \\
\frac{q_{p r}}{\vec{q}_{p}}
\end{array}, \frac{\vec{q}_{p}}{q_{p}}
\end{aligned}
$$

we have

$$
T_{p o l}=\left\langle\chi_{\vec{q}_{p}}^{c(-)}\left(\vec{r}_{d A}\right) e^{i\left(\vec{Q}_{r} \vec{r}_{n p}+\vec{q}_{n} \vec{r}_{d A}\right)}\left|V_{n p}\left(\vec{r}_{n p}\right)\right|^{c} \chi_{\vec{q}_{d}^{(+)}}^{\left(\vec{r}_{d A}\right)} \tilde{\Phi}_{d}\left(\vec{r}_{d A}, \vec{r}_{n p}\right)\right\rangle,
$$


with

$$
\vec{Q}_{r}=\frac{1}{2}\left(\vec{q}_{n}-\vec{q}_{p r}\right) \text {. }
$$

Evaluation of eq. (5.10) gives :

$$
\begin{aligned}
& T_{p o l}=-i \frac{Z e^{2}}{\hbar^{2}} m_{n p} \frac{(4 \pi)^{3 / 2}}{3} A_{0}\left(Q_{r}\right) I_{p}\left(Q_{r}\right) . \\
& \sum_{m} Y_{1 m}^{*}\left(\hat{q}_{n}\right) \int d^{3} r_{d A}{ }^{c} X_{\vec{q}_{p}}^{(-) *}\left(\vec{r}_{d A}\right) e^{-i \vec{q}_{n} \vec{r}_{d A}} \frac{Y_{1 m}\left(\hat{r}_{d A}\right) c}{r_{d A}^{2}} X_{\vec{q}_{d}}^{(+)}\left(\vec{r}_{d A}\right) .
\end{aligned}
$$

Hereby we have introduced

$$
I_{p}\left(Q_{r}\right)=\left(\beta^{2}+Q_{r}^{2}\right) \int_{0}^{\infty} d r r^{2} j_{1}\left(Q_{r} r\right) \frac{e^{-\mu_{0} r}}{1-e^{-\mu_{0} r}} g_{d}(r) .
$$

This integral can be calculated analytically leading to

$$
\begin{gathered}
I_{p}\left(Q_{r}\right)=\left(\beta^{2}+Q_{r}^{2}\right)\left\{\sum _ { n = 1 } ^ { \infty } \left(\frac{1}{4 \alpha} J_{3}\left(\alpha+n \mu_{0}\right)+\frac{1}{\mu_{0}(\beta+\alpha)^{2}}\left(2 J_{1}\left(\beta+n \mu_{0}\right)\right.\right.\right. \\
\left.\left.\left.+(\beta+\alpha) J_{2}\left(\beta+n \mu_{0}\right)\right)\right)-\frac{2}{\mu_{0}^{2}(\beta+\alpha)^{2}}\left(J_{0}(\beta)+\alpha J_{1}(\beta)\right)\right\} .
\end{gathered}
$$

The integrals

$$
Y_{\lambda}(x)=\int_{0}^{\infty} d r j_{1}\left(Q_{r} r\right) e^{-x r} r^{\lambda},
$$

can easily be expressed by elementary functions $[77]$. Expanding eq. $(5.14)$ in $(\alpha / \beta)$ we get

$$
\begin{aligned}
I_{p}\left(Q_{r}\right) & \simeq \frac{2 Q_{r} \beta+\frac{1}{4} Q_{r} \alpha+\frac{1}{20}\left(\beta Q_{r}\right)^{3 / 2}}{\alpha\left(\beta^{2}+Q_{r}^{2}\right)^{2}} \\
& \simeq \frac{2 Q_{r} \beta}{\alpha\left(\beta^{2}+Q_{r}^{2}\right)^{2}} .
\end{aligned}
$$


As is shown in fig. 13 the approximation (5.16) is valid in the energy-region of interest within $0,5 \%$.

Assuming that the main contribution to the radial integral (5.12) comes from the vicinity of the classical turning point, the influence of the polarization can be estimated by

$$
\Delta T=\frac{T_{f i}^{(+)}-T_{p c}}{T_{p c}} \simeq-i \frac{2 m_{n p}}{\hbar^{2}} \frac{E_{d}^{2}}{z e^{2}} \frac{\beta Q_{r}}{\alpha\left(\beta^{2}+Q_{r}^{2}\right)^{2}} \frac{\vec{q}_{p} \vec{q}_{n}}{q_{p} q_{n}} .
$$

Since in the no-recoil approximation $q_{p r}=0$, and thus $\vec{Q}_{r}=\frac{\vec{q}_{n}}{2}$, we easily find that in break-up reactions on heavy nuclei the contributions of the polarization are always smaller than $\sim 18$. To calculate this effect more precisely we can perform a partial wave expansion of eq. (5.12) leading to

$$
\begin{gathered}
T_{p o l}=-i z e^{2} \frac{2 m_{n p}}{\hbar^{2}} A_{0}\left(Q_{r}\right) I_{p}\left(Q_{r}\right)(4 \pi)^{5 / 2} \sum_{l_{d} \ln l_{p}} i^{l_{d}-l_{n}-l_{p}} e^{i\left(\sigma_{l d}\left(\eta_{d}\right)+\sigma_{l p}\left(\eta_{p}\right)\right)} \hat{l}_{d}^{2} \hat{l}_{p} \\
G_{l_{\alpha} l_{n} l_{p}}\left(\theta_{p}, \theta_{n}, \Phi\right) R_{l_{d} l_{n} l_{p},}^{p o l},
\end{gathered}
$$

where

$$
\begin{aligned}
& G_{l d l n}\left(\theta_{p}, \theta_{n}, \Phi\right)= \frac{(4 \pi)^{3 / 2}}{3 \hat{l_{d}} \hat{l}_{p}} \sum_{m m_{n} m_{p}} Y_{1 m}^{*}\left(\theta_{n}, \Phi\right) Y_{l_{n} m_{n}}^{*}\left(\theta_{n}, \Phi\right) Y_{l_{p} m_{p}}^{*}\left(\theta_{p}, 0\right) \\
& \cdot \int d \Omega Y_{l_{d} 0}(\Omega) Y_{i_{p} m_{p}}(\Omega) Y_{1 m}(\Omega) Y_{i_{n} m_{n}}(\Omega) \\
&=\hat{l}_{n}^{2} \sum_{l_{m}} \hat{\imath} Y_{i_{p},-m}\left(\theta_{p}, 0\right) Y_{l_{n} m}\left(\theta_{n}, \Phi\right)\left(\begin{array}{ccc}
l & l_{\alpha} & l_{p} \\
-m & 0 & m
\end{array}\right)\left(\begin{array}{lll}
l & l_{d} & l_{p} \\
0 & 0 & 0
\end{array}\right)\left(\begin{array}{lll}
1 & l_{n} & l \\
0 & 0 & 0
\end{array}\right)^{2},
\end{aligned}
$$

and

$$
R_{l_{d} l_{n} l_{p}}^{p o l}=\frac{1}{q_{d} q_{p}} \int_{0}^{\infty} d r F_{l_{d}}\left(\eta_{d}, q_{d} r\right) \frac{\dot{j}_{n}\left(q_{n} r\right)}{r^{2}} F_{l_{p}}\left(\eta_{p}, q_{p} r\right) .
$$


Since the radial integral $R_{l_{d} l_{n} l_{p}}^{p o l}$ can easily be calculated using the methods outlined above, expression (5.19) is well suited for a numerical treatment.

In figs. 14, 15 we show some model calculations in the no-recoil approximation based on eq. (5.18) for different angles and energies. As can be seen the polarization is very small and these calculations are in good agreement with the approximate formula $(5.17)$.

Now we want to see if this small polarization effect can be simulated by the ansatz described above. Therefore, we have compared the pure coulomb break-up cross section (eq. (4.56) with $\left.O_{j n l_{n}} \equiv 0\right)$ with (5.5). The two six-dimensional integrals occurring on the right-hand side of eq. (5.5) (c.f. eq. (3.28) and eq. (5.6) ) were now calculated by using the LEA-method. Defining the multiplication factor $t_{r}$ by

$$
\vec{q}_{p r}=t_{r} \cdot \vec{q}_{p}
$$

we find from eq. (5.8) that $t_{r} \simeq 0.25-0.75$ in the sub-Coulomb energy region. The results of fig. 16 where we have again plotted the relative deviations of the cross sections show that indeed a very good agreement between the two T-matrices eqs. $(4.56,5.5)$ can be achieved if the recoil ist taken approximately into account in the LEA with $t_{r} \simeq 0.5$.

In conclusion we have the result that the polarization of the deuteron is a small effect and can be approximately described by the ansatz of Dar et al. [84]. 


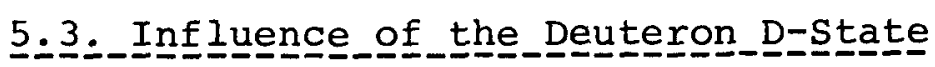

At a first glance the D-state of the deuteron seems to lead to the most important correction compared to the other "small effects". The deuteron in the loosely bound D-state might dissociate more easily than in the s-state.

Having established the high accuracy of the sub-Coulomb T-matrix (4.56) this effect can be calculated immediately. Inserting the general form factor (4.41) in (4.38) we obtain in the sub-Coulomb energy region:

$$
T_{f i}^{(t)}=(4 \pi)^{3 / 2}\left(A_{0}\left(q_{n}\right)+A_{2}\left(q_{n}\right)\right)\left\{M_{p c}+\sum_{l n} O_{j n l n} T_{l_{n}}^{n}\right\} .
$$

For the coincidence cross section we get the result

$$
\left(\frac{d^{3} \sigma}{d \Omega_{p} d \Omega_{n} d E_{n}}\right)_{s+D}=\left(\frac{d^{3} \sigma}{d \Omega_{p} d \Omega_{n} d E_{n}}\right)_{s}\left(1+\left(\frac{A_{2}\left(q_{n}\right)}{A_{0}\left(q_{n}\right)}\right)^{2}\right) .
$$

Therefore, including the D-state of the deuteron leads only to a simple overall shift of the cross section. This is in agreement with corresponding results in bound state stripping reactions $[105,106]$.

Naturally, the incoherence of the S- and D-state contributions is a consequence of the neglect of any spin-orbit interaction. At higher energies where such interactions are not negligible both contributions have to be added coherently. 
Since now the influence of the deuteron $D$-state has been reduced to the simple factor in eq. (5.23) we will calculate the ratio $A_{2}\left(q_{n}\right) / A_{0}\left(q_{n}\right)$ by using the internal wave functions of the deuteron. (The integrals $H_{L}\left(q_{n}\right)$ are formally equal to the corresponding expressions defined by Johnson and Santos in ref.[106] • We will therefore closely follow their treatment).

Expanding $A_{L}\left(q_{n}\right)$ given in eq. (4.44) about $q_{n}=0$ we obtain

$$
A_{0}\left(q_{n}\right)=\frac{D_{0}}{\sqrt{4 \pi}}\left(1-o\left(\frac{g_{n}}{\beta}\right)^{2}\right) \text {, }
$$

with

$$
D_{0}=-\frac{\hbar^{2}}{2 m_{n p}} \sqrt{4 \pi}\left(\left(\alpha^{2}+q_{n}^{2}\right) u_{0}\left(q_{n}\right)\right)_{q_{n}=0}=-\frac{B_{d}}{\sqrt{4 \pi}} \int_{0}^{\infty} d r u_{0}(r) .
$$

This zero-range constant can be rewritten as

$$
D_{0}=D_{0}^{0} \frac{N \cos \varepsilon_{g}}{\sqrt{2 \alpha}}\left(1-L_{0}\right)
$$

with

$$
L_{0}=\alpha^{2} \int_{0}^{\infty} d r r^{2}\left(e^{-\alpha r}-\frac{r u_{0}(r)}{N \cos \varepsilon g}\right) \text {. }
$$

Using the Hulthen description of the deuteron yields the usual zero-range constant eq. (4.11). For the"D-state integral"we have

$$
A_{2}\left(q_{n}\right)=\frac{D_{2} D_{0}}{\sqrt{4 \bar{x}}} q_{n}^{2}\left(1+o\left(\frac{q_{n}}{\beta}\right)^{2}\right)
$$


with

$$
\begin{aligned}
D_{2} & =\lim _{q_{n} \rightarrow 0}\left(q_{n}^{2} U_{0}\left(q_{n}\right)\right)^{-1} U_{2}\left(q_{n}\right) \\
& =\alpha^{-2} \tan \varepsilon_{g}\left(1-L_{2}\right)\left(1-L_{0}\right)^{-1}
\end{aligned}
$$

and

$$
L_{2}=\frac{\alpha^{5}}{15} \int_{0}^{\infty} d r r^{4}\left(\left|h_{2}^{(t)}(i \alpha r)\right|-\frac{u_{2}(r)}{N \sin \varepsilon_{g}}\right) .
$$

The constant $\mathrm{D}_{2}$ is roughly related to the quadrupole moment $Q_{D}$ of the deuteron by

$$
D_{2} \simeq \sqrt{2} Q_{D}=0.39 \mathrm{fm}^{2}
$$

A calculation of $D_{2}$ using McGee's wave function gives [106] $\mathrm{D}_{2}=0.488 \mathrm{fm}^{2}$. Using eqs. $(5.24)$ and (5.28) leads to :

$$
\frac{A_{2}\left(q_{n}\right)}{A_{0}\left(q_{n}\right)} \simeq D_{2} q_{n}^{2} \simeq 0.5 q_{n}^{2}
$$

In order to check this approximate formula we have calculated this ratio exactly by using the Hulthèn wave functions given in eq. (3.18). As can be seen in fig. 17, (5.32) is valid up to large values of $q_{n}\left(E_{n}\right)$. Furthermore, the ratio is quite insensitive to different values of the hard core radius $r_{c}$ and the D-state probability $P_{D}$.

In the energy region of interest, $q_{n}$ is quite small $\left(q_{n} \leqslant 0.2\right)$ and therefore the D-state can be almost neglected, giving only a 18 shift of the cross section in the most infavourable case. 
While at energies above the coulomb barrier this simple treatment of the D-state is no longer valid, it seems reasonable that even there the D-state has little influence.

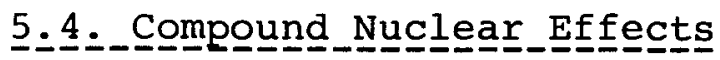

The energy of the break-up neutrons lies in a range form zero to several MeV. It is well known that in this energy region compound nucleus (CN) formation plays an important role. Here we want to estimate compoñd nuclear effects on the break-up cross section. We write as usual the S-matrix of the neutronnucleus elastic scattering as a sum of an energy averaged part $\left\langle S_{i_{n}}\right\rangle$, described by an appropriate opticalfnodel and a fluctuating part $F_{l_{n}}$ (with $\left\langle F_{l_{n}}\right\rangle=0$ ):

$$
S_{l_{n}}=\left\langle S_{l_{n}}\right\rangle+F_{l_{n}} \text {. }
$$

Then we get $[30] *$ :

$$
\begin{aligned}
\frac{d^{3} \sigma}{d \Omega_{p} d \Omega_{n} d E_{n}} & \sim\left|M_{p c}+\frac{1}{2} \sum_{l_{n}}\left(\left\langle S_{l_{n}}\right\rangle-1\right) T_{l_{n}}^{n}\right|^{2} \\
& +\frac{1}{4} \sum_{l_{n} l_{n}^{\prime}}\left\langle F_{l n} F_{l_{n}^{\prime}}^{*}\right\rangle T_{l_{n}}^{n} T_{l_{n}^{\prime}}^{n *} .
\end{aligned}
$$

Therefore, the CN contributions can easily be calculated by applying the Hauser-Feshbach-Wolfenstein theory $[107,108]$.

*) Note that the fluctuating part drops out in the interference term. 
The statistical assưptions of this theory are generally fulfilled for heavy nuclei and neutrons of several MeV. Using the results of ref. [109], we can write in the absence of direct reactions and for small absorption:

$$
\left\langle F_{l \iota}^{\iota} F_{\iota^{\prime} \iota^{\prime}}^{\iota^{\prime}}\right\rangle=\frac{t_{\iota} t_{\iota^{\prime}}}{\sum_{l^{\prime \prime}} t_{\iota^{\prime \prime}}} W_{\iota^{\prime}} \delta_{l l^{\prime}} .
$$

Here we have relabelled the fluctuating part of the scattering matrix as $F^{J} c^{\prime}$ ', where $c$ describes a channel for the formation of a compound system with spin and parity denoted by $J$, and $c^{\prime}$ describes one of the decay channels. Furthermore, we have limited ourselves to a zero-spin target, for simplicity's sake. The transmission coefficients $t_{l}$ in (5.35) are given by

$$
t_{L}=1-\left|S_{i}\right|^{2}
$$

and the quantity $W_{l^{\prime}}$ is the usual level width fluctuation correction. The sum over $l^{\prime \prime}$ is performed over all open channels for the decay of a compound nucleus state with spin $l$ and parity $(\leftarrow)^{l}$. Furthermore, we have assumed that there are no level-level and channel-channel correlations.

Finally, we can write for the positive constribution of the fluctuating part:

$$
\begin{aligned}
\frac{1}{4} \sum_{\ln l^{\prime}}\left\langle F_{\ln } F_{l n}^{*}\right\rangle & T_{\ln }^{n} T_{l n}^{n}{ }^{*} \\
& =\frac{1}{4} \sum_{\ln } \frac{t_{\ln }^{2}\left|T_{l n}^{n}\right|^{2}}{\sum_{l_{n}^{\prime \prime}} t_{l n}^{\prime \prime}} W_{l n} l_{n} .
\end{aligned}
$$

In figs. 18, 19 we have shown the CN-contributions for the reaction ${ }^{208} \mathrm{~Pb}(\mathrm{~d}, \mathrm{pn}){ }^{208} \mathrm{~Pb}$. As can be seen for $\theta_{n}=40^{\circ}, \theta_{p}=600$ the pure coulomb term dominates and the contribution of the $\mathrm{CN}$ is very small in this case. On the other hand, for $\theta_{n}=35^{\circ}$, 
$\theta_{p}=-60^{\circ}$ the pure coulomb term is relatively small and nuclear interactions are dominant. Therefore, as expected, the CN effects show up in this case. Although the agreement between our theory and the experimental result is good, more accurate data in the lead region are needed to justify a detailed comparison of the theory with experiment.

\section{Semiclassical Model}

For sub-Coulomb stripping reactions to bound states a simple semiclassical description has been shown to work very well $[94]$. Therefore, it is tempting to use such a description [110] also for our sub-Coulomb theory of break-up reactions. Due to the close analogy of the two processes, it is easy to adapt the semiclassical formalism describing bound state stripping to include break-up reactions.

Using the method developed in refs. $[111,74,94,110]$ the results of the semiclassical approximation can be written in the so-called focal coordinate system as

$$
\begin{gathered}
M_{l_{n} m_{n}}\left(\theta_{p}\right)=\int d^{3} r{ }^{c} X_{\vec{q}_{p}}^{(-) *}(\vec{r}) f_{j n l_{n}}\left(q_{n}, r\right) Y_{l_{n} m_{n}}^{*}(\hat{r}){ }^{c} X_{\vec{q}_{d}}^{(t)}(\vec{r}) \\
\simeq \frac{4 \pi i}{Q q_{n}} \bar{f}_{c}\left(\theta_{p}\right) Y_{\ln m_{n}}\left(\frac{\pi}{2}, \bar{r}-\theta_{p}\right) I_{\ln m_{n}}\left(\varepsilon\left(\theta_{p}\right), \xi_{,}, \xi^{\prime}, \rho\right) .
\end{gathered}
$$

Here the semiclassical integral $I_{l_{n} m_{n}}$ is the same one as defined in eq. (4.92) except that the Hankel function in the integrand has to be replaced by the full radial neutron wave function. In the sub-Coulomb energy region we can use (4.35). In- 
troducing the integral $I_{\ln m_{n}}^{0}$ obtained from (4.92) by replacing the Hankel function by a Bessel function, i.e.

$$
I_{\ln m n}^{0}=\operatorname{Re}\left(I_{\ln m_{n}}^{+}\right)
$$

we get

$$
I_{\ln m n}=I_{\ln m n}^{0}+0_{j n n} I_{l_{n} m_{n}}^{+} .
$$

The semiclassical limit (6.1) can be most easily obtained from the DWBA expression by using the partial wave expansion (4.71) with the semiquantal prescription for the radial integrals. The sum over the partial waves is approximated by an integral where the spherical harmonics and the Clebsch-Gordan coefficients are replaced by their asymptotic values corresponding to large values of the angular momentum. This integral is then evaluated by using the saddle point approximation. (For a detailed description of this procedure we refer the reader to the literature $[74,94,111]$ ).

The semiclassical expression (6.1) can be derived also by using a wave packet description of the process. As was shown by Broglia and Winther $[112]$ these wave packets then move on a mean classical orbit, averaged over the initial and final trajectories, where the eccentricity $\in$ is connected with the scattering angle of the proton by

$$
\frac{1}{\varepsilon}=\sin \frac{\theta_{\mathrm{p}}}{2}
$$

Finally, the symmetrized Rutherford scattering amplitude in $(6.1)$ is given by 


$$
\bar{f}_{c}\left(\theta_{p}\right)=-\frac{a_{c}}{2} \varepsilon^{2} \exp \left\{2 i\left(\sigma_{0}(\eta)+\eta \log \varepsilon\right)\right\} .
$$

Transforming expression (6.1) from the focal coordinate system to the incident beam coordinate system we imnediately obtain for the differential cross section :

$$
\begin{aligned}
& \frac{d^{3} \sigma}{d l_{p} d \Omega_{n} d E_{n}}=4\left(\frac{2}{\hbar^{2}}\right)^{3} \frac{\mu d \mu_{p} m_{n}}{Q^{2} q_{n}} \frac{q_{p}}{q_{d}}\left|A_{0}\right|^{2}\left|\bar{f}_{c}\right|^{2} . \\
& \cdot \mid \sum_{\ln m_{n}} i^{-\ln } Y_{l_{n} m_{n}}^{*}\left(\theta_{n}, \Phi\right) \sum_{m} Y_{l_{n},-m}\left(\frac{\pi}{2}, 0\right) D_{-m_{n}, m}^{l_{n}}\left(\frac{\pi}{2}, \frac{\pi}{2}, \frac{\pi+\theta_{p}}{2}\right) \\
& \left.\cdot I_{\ln m}\left(\varepsilon, \xi, \xi^{\prime}, \rho\right)\right|^{2}
\end{aligned}
$$

This expression can be further simplified by introducing a rotation

$$
Y_{\lambda \mu}(\alpha, \beta)=\sum_{m} Y_{\lambda m}\left(\theta_{n}, \frac{\pi}{2}-\Phi\right) d_{m \mu}^{\lambda}\left(\frac{\pi}{2}\right)
$$

with

$$
\begin{aligned}
& \cos \alpha=-\sin \theta_{n} \sin \Phi \\
& \sin \alpha \cos \beta=\cos \theta_{n}, \quad \sin \alpha \sin \beta=\sin \theta_{n} \cos \Phi
\end{aligned}
$$

Using eq. (6.7) we get :

$$
\begin{aligned}
& \frac{d^{3} \sigma}{d \Omega_{p} d \Omega_{n} d E_{n}}=4\left(\frac{2}{\hbar^{2}}\right)^{3} \frac{\mu_{d} \mu_{p} m_{n}}{Q^{2} q_{n}} \frac{q_{p}}{q_{d}}\left|A_{0}\right|^{2}\left|\bar{f}_{c}\right|^{2} \\
& \cdot\left|\sum_{\ln m_{n}} i^{\ln } Y_{l_{n} m_{n}}(\alpha, \beta) Y_{l_{n} m_{n}}\left(\frac{\pi}{2}, \frac{\pi-\theta_{p}}{2}\right) I_{\ln m n}\left(\varepsilon, \xi, \xi^{\prime}, \rho\right)\right|^{2}
\end{aligned}
$$

This is our final result describing the break-up of the deuteron in the semiclassical approximation. We note that the semiclassical description only refers to the charged particles in the entrance and exit channels, while the neutron in the continuum is still treated quantally. 
Before applying the semiclassical method to experimental data it should be clarified whether or not it gives an accurate description of the reaction as compared to the exact quantal treatment described above. In ref. $[113]$ it was shown that indeed the agreement between the semiclassical and quantal expressions is surprisingly good. As an example we show in fig. 20 the deviation $E\left(\theta, \xi, \xi^{\prime}, \rho, \eta\right)$ of the semiclassical cross section from the quantum-mechanical break-up cross section as a function of the coulomb parameter $\eta$ for several values of $\rho$. In general, the difference between both methods is less than $1 \% 2 \%$. The dependence of this difference on the other parameters is rather small as was shown in ref. [113]. At a first glance this may seem surprising, because e.g. the relative change of mass during the reaction is $\frac{\Delta m}{m}=\frac{1}{2}$ and not, as had to be assumed in ref. [112] much smaller than 1. But since the relative changes of the other parameters ( $E$ and $\zeta$ ) are also quite noticeable, all these effects may roughly compensate so that the notion of an averaged trajectory is still essentially correct. Moreover, if such a mean classical orbit is not a good approximation, i.e. if the errors of the semiclassical description may be large, the cross section is very small and therefore the large errors in the semiclassical treatment become difficult to observe.

More formally, the high accuracy of the semiclassical method for break-up reactions can be well understood by applying the "redistribution theorem" of Breit and Daitch $[114]$. This theorem states that the quantity $\tau=\frac{\rho}{\eta}$ is a measure for the accuracy of the semiclassical method. Although $\eta$ in our case is only about 5 , 
the parameter $\rho$ connected with the neutron energy in the continuum is rather small at the maximum cross section where $E_{n} \approx 0$. Therefore, $\tau$ is also small and the semiclassical approximation works well (for further details see ref. [113]). Indeed, a recalculation of the coincidence cross sections shown in figs. 7 - 10, using the semiclassical model, gives practically the same results as the quantal treatment (cf. ref. [113]). The accuracy of the semiclassical treatment becomes even better if integrated cross sections are considered. This is shown e.g. in fig. 21 for the model calculation ${ }^{208} \mathrm{~Pb}(d, p n)^{208} \mathrm{~Pb}$, where the neutron is not observed. For this case we obtain from eq. (6.9) by integrating over $\theta_{n}, \Phi$ :

$$
\begin{aligned}
\frac{d^{2} \sigma}{d \Omega_{p} d E_{n}}= & 4\left(\frac{2}{\hbar^{2}}\right)^{3} \frac{\mu_{d} \mu_{p} m_{n}}{Q^{2} q_{n}} \frac{q_{p}}{q_{d}}\left|H_{0}\right|^{2}\left|\bar{f}_{c}\right|^{2} . \\
& \cdot \sum_{\ln m_{n}}\left|Y_{\ln m_{n}}\left(\frac{\pi}{2}, 0\right) I_{\ln m_{n}}\left(\varepsilon, \xi, \xi^{\prime}, \rho\right)\right|^{2} .
\end{aligned}
$$

This very simple expression allows a fast calculation of this cross section and those obtained from (6.10) by integrating over angle or energy. In figs. 22 - 25 we have shown some examples for such model calculations. A quantal treatment of these cross sections - leading practically to the same results needs much more computing time.

We applied our semiclassical model only to the sub-Coulomb energy region. Above the Coulomb barrier the well-known difficulties of describing the imaginary part of the optical potential classically may destroy the simplicity and accuracy of the semiclassical approximation. 
Beside the numerical advantages of the semiclassical method, it gives us a simple classical picture of the break-up reaction : the charged particles are moving on classical orbits while the neutron is "radiated off" along the trajectory. This classical idea is also in good qualitative agreement with the conclusions which can be drawn from fig. 24 : from the most probable proton (neutron) energy $E_{p}^{\text {opt }}\left(E_{n}^{\text {opt }}\right)$ one can determine a "break-up radius" according to rather crude classical arguments, based on energy considerations, given in ref. $[18]$ as

$$
R_{B}=\frac{Z e^{2}}{E_{d}-B_{d}-2 E_{n}^{o p t}}
$$

This should be approximately equal to the average classical turning point

$$
R_{c l}=a_{c}(1+\varepsilon)
$$

Indeed, for $\mathrm{Ed}=9 \mathrm{MeV}$ we find with $E_{n}^{\text {opt }} \simeq 0.75 \mathrm{MeV}$, that $R_{B} \simeq 22 \mathrm{fm} \simeq R_{C L} \simeq 18 \mathrm{fm}$.

\section{Stripping into the continuum}

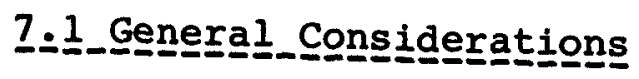

The theory of deuteron break-up developed above lends itself in a natural way to a treatment of stripping reactions into the continuum (unbound states). Indeed, such processes are in most cases practically the same asabreak-up reaction, the presence of an unbound state or a resonance can straightforwardly be accounted 
for. According to wigner $[115]$, the rapid variation with energy in the phase shift of the neutron target scattering corresponds to a time delay, i.e. an intermediate state $B=(A+n)_{\text {res }}$ is formed for a certain time.

As discussed above we mean by a stripping reaction into the continuum the process

$$
\alpha+A \rightarrow p+\text { anything, }
$$

where the decay modes of the subsystem $B=A+n$ are not detected. We require that the energy of the system $B$ is above the threshold for neutron emission*).

Since only particle $p$ is detected, we have to sum over all decay channels of $B$. This corresponds to the type of "inclusive reactions" well-known in high energy physics.

Here, we are especially interested in the direct reaction mechanism, where the wave function of the transferred particle with respect to the target nucleus plays the key role. In bound state stripping this essentially means that all the many body aspects of the process are hidden in the phenomenologically

*) Below that threshold, the usual DWBA formalism [4] applies. of course, even in this case of stripping to a bound state, the system can still be unstable with respect to emission of other particles than the transferred one, but this is considered irrelevant for the usual DWBA formalism (e.g. the reaction $7_{\mathrm{Li}}(\mathrm{d}, \mathrm{n}) 8_{\mathrm{Be}}$, where $8_{\mathrm{Be}}$ is unstable with respect to decay into two $\alpha$-particles, but stable with respect to decay into $\left.p+{ }^{7} \mathrm{Li}\right)$. 
introduced spectroscopic factors which are determined in practice by fitting a theoretical angular distribution to experiment. In the case of stripping to unbound states the relevant quantity will be the phase shift of neutron-target elastic scattering, which has a direct physical meaning (see also ref. $[116]$ ).

\section{2-2_Theor $y$}

Let us first consider explicitly the case where the only open decay channel of $B$ is back to $A$ (groundstate) $+n$. Already there most of the important features can be observed. In this case we can immediately apply our formalism of d-break-up where we assumed $A$ to be inert. The result for the cross section in the general multichannel case is proportional to $\Gamma_{n}$, the neutron partial width of that resonance, as was shown in ref. [116]. It does not depend on the fact that $B$ may decay into channels other than $A+n$. In this chapter we want to discuss important limiting cases of the break-up amplitude. Starting from the formula for the cross section of the kinematically complete process $A+d \rightarrow A+p+n$ we obtain the cross section $d^{2} \sigma / d \varepsilon_{p} d E_{n}$ for the inclusive type of reaction $A+d \rightarrow p+$ anything by integrating over the angle of the unobserved neutron, since there are no other channels present in this model. To avoid further complications we introduce the zero-range approximation for the moment and assume that the radial neutron wave function $(3.14)$ depends only on $j_{n}$ and $l_{n}$. 
We have

$$
\begin{aligned}
& \frac{d^{2} \sigma}{d \Omega_{p} d E_{n}} \equiv \int d \Omega_{n} \frac{d^{3} \sigma}{d \Omega_{p} d \Omega_{n} d E_{n}} \\
& =\frac{\mu_{d} \mu_{p} m_{n}}{4\left(\pi \hbar^{2}\right)^{3}} q_{q_{d} q_{n}} \frac{2 J_{B}+1}{2 J_{n}+1} D_{0}^{2} \sum_{j n l_{n} m_{n}} \frac{1}{2 \ln _{n}+1}\left|M_{j n l_{n} m_{n}}\right|^{2},
\end{aligned}
$$

with

$$
M_{j n \ln m_{n}}=\int d^{3} r X_{\vec{q}_{p}}^{(-) *}(\vec{r}) f_{j n l_{n}}(r) Y_{i_{n} m_{n}}(\hat{r}) X_{\overrightarrow{q_{d}}}^{(t)}(\vec{r}) .
$$

The radial part of the radial neutron wave function is denoted again by $f_{j_{n} l_{n}}(r)$. Here we find it convenient to divide it into three parts :

$$
\begin{aligned}
\hat{f}_{j_{n} l_{n}}(r) & =\theta\left(R_{n}-r\right) u_{j_{n} l_{n}}(r)+ \\
& +\theta\left(r-R_{n}\right)\left\{j_{l_{n}}\left(q_{n} r\right)+O_{j_{n} l_{n}} h_{l_{n}}^{(r)}\left(q_{n} r\right)\right\},
\end{aligned}
$$

where $R_{n}$ denotes the distance for which the nuclear potential has become negligibly small. The first term denotes the wave function inside the nuclear potential (see below). The second term denotes the free wave, the third term is the scattered wave which depends only on the phase shift of the neutronnucleus elastic scattering. According to this decomposition we split the T-matrix into three parts :

$$
M_{j n \ln m n}=M_{j n l_{n} m_{n}}^{L}+M_{l n m_{n}}^{0}+o_{j n l n} M_{l n m_{n}}^{+} .
$$

Naturally, in general all three terms will be present, but it is interesting to examine qualitatively various limiting cases, where one or the other term is dominant. 


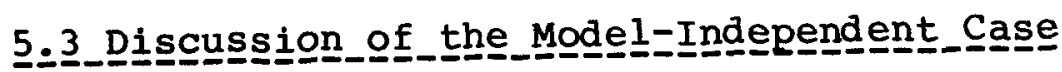

In the case of sub-Coulomb transfer reactions or heavy ion transfer reactions generally characterized by strong absorption the extreme smallness of the distorted waves in the nuclear interior makes the contribution due to $M_{j n \ln m_{k}}^{<}$small. Therefore, we can neglect it and we are left with a discussion of the second and the third term, which depends only on the modelindependent phase shifts.

The relative importance of $M_{l_{n} m_{n}}^{0}$ and $M_{l_{n} m_{n}}^{+}$given by the quantity $V_{i_{n} m_{n}}=\left|\frac{M_{i n m_{n}}^{0}}{M_{i_{n} m_{n}}^{t}}\right|$, will depend on the ratio of $j_{l_{n}}$ and $h_{l_{n}}^{(t)}$ for those $r$-values which contribute most to the distorted wave matrix element and on the "degree of distortion" of the initial and final state scattering wave functions $\chi_{\vec{q}_{d}}^{(+)}$and $\chi_{\vec{q}_{p}}^{(-) *}$.* In the plane wave limit, it is easy to see that $M_{l_{n} m_{n}}^{0}=0$ (for $R_{n}=0$ ) because $\vec{q}_{d}=\vec{q}_{p}+\vec{q}_{n}$, which follows from energy and momentum conservation. Therefore, we have :

$$
\begin{aligned}
& M_{l_{n} m_{n}}^{0}=\int d^{3} r e^{i \vec{q}_{d p} \vec{r}} j_{i_{n}}\left(q_{n} r\right) Y_{l_{n} m_{n}}(\hat{r}) \\
& =4 \pi i^{l_{n}} Y_{l_{n} m_{n}}\left(\hat{q}_{d p}\right) \int_{0}^{\infty} d r r^{2} j_{l_{n}}\left(q_{d p} r\right) j_{l_{n}}\left(q_{n} r\right)=0,
\end{aligned}
$$

where

$$
\vec{q}_{d p}=\vec{q}_{d}-\vec{q}_{p}
$$

Despite its unphysical features it seems instructive to study the case of pure Coulomb distortion. The charge $z$ of the target nucleus measures the"degree of distortion". Treatingonly

*) Note that in the case of a resonance, the phase shift goes through $\pi / 2$, therefore $O_{j l n}$ is of order unity in the region of a resonance. 
the case $l_{n}=m_{n}=0$ we can use the analytical formula eq. (4.60) which leads to

$$
V_{00}=\left|\frac{M_{00}^{0}}{M_{00}^{+}}\right|=\frac{1}{2}\left|1-e^{i \gamma}\right|=\left|\sin \frac{\gamma}{2}\right|,
$$

with $\gamma$ defined in eq. $(4.69)$.

In fig. 26 the ratio $V_{00}$ is given for $E_{d}=10 \mathrm{MeV}$ and $12.5 \mathrm{MeV}$, respectively, as a function of $z$ for various values of $E_{\eta}$. Voo is zero for $\mathrm{z}=0$ and increases with $\mathrm{z}$, later on $1 \mathrm{t}$ oscillates. It is expected that for other values of $l_{n}$ the zeroes of $V_{i_{n} m_{n}}$ lie at different values of $z$. Of course, in realistic cases, nuclear distortion has to be taken into account and in general the ratio $V_{l_{n} m_{n}}$ shows a kind of "penetration behaviour" : for large values of $x=q_{n} r$ it will tend to unity, whereas for $x \ll 1$ we have the expansion

$$
\frac{\dot{j}_{n}(x)}{n_{l_{n}}(x)} \simeq-\frac{x^{\ln }}{\left(2 l_{n}+1\right) ! !} \frac{x^{l_{n}+1}}{\left(2 l_{n}-1\right) ! !}=-\frac{x^{2 \ln +1}}{\left(2 l_{n}-1\right) ! !\left(2 l_{n}+1\right) ! !} .
$$

From this it follows that for small values of $x$ (corresponding to low neutron energy) and large values of $l_{n}, M_{l_{n} m_{n}}^{+}$is completely dominant over $M_{l_{n} m_{n}}^{0}$. Therefore, the scattered term dominates, and we have for the partial cross section :

$$
\begin{aligned}
\frac{d^{2} \sigma_{j n i n}}{d \Omega_{p} d E_{n}} & \sim \sin ^{2} \delta_{j n l x} \sum_{m_{n}}\left|M_{l_{n} m_{n}}^{+}\right|^{2} \\
& \sim \sigma_{j n l_{n}} \sum_{m_{n}}\left|M_{l_{n} m_{n}}^{+}\right|^{2} .
\end{aligned}
$$


This formula exhibits the "parallelism" of the $(d, p)$ cross section with total neutron elastic scattering, as was first observed by Fuchs et al. $[34,35]$ in the reaction ${ }^{15} \mathrm{~N}(\mathrm{~d}, \mathrm{p}){ }^{16} \mathrm{~N}$ (unbound). Later on this parallelism was tested by Huby et al. $[117,118]$ for the reaction ${ }^{12} \mathrm{c}(\mathrm{d}, \mathrm{p}){ }^{13} \mathrm{C}$ (unbound). Analyzing experimental data of Darden et al. $[58]$ they found that relation (7.10) is indeed very well fulfilled.

It is very interesting to note that high $l_{n}$-values are weighted more in a stripping reaction as compared to elastic neutron scattering. This is easy to understand physically, because the neutron is transported through the centrifugal barrier by the deuteron with high energy. This fact can be expressed by introducing the "stripping enhancement factor" $[37]$ in eq. (7.10): $\frac{d^{2} \sigma_{j n l n}}{d \Omega_{p} d E_{n}}=\sigma_{j n l_{n}} \cdot F_{i_{n}}\left(q_{n} ; \vec{q}_{d}, \vec{q}_{p}\right)$,

with

$$
F_{l n}\left(q_{n} ; \vec{q}_{d}, \vec{q}_{p}\right)=\frac{\mu_{d} \mu_{p}}{\pi\left(2 \pi \hbar^{2}\right)^{3}} \frac{q_{p}}{q_{d}} q_{n}^{3} m_{n} \frac{D_{0}^{2}}{2 l_{n}+1} \sum_{m_{n}}\left|M_{\ln m_{n}}^{+}\right|^{2} .
$$

To get a qualitative feeling for the behaviour of this stripping enhancement factor we may express it analytically after introducing plane waves for $\chi_{\vec{q}_{d}}^{(t)}, \chi_{\vec{q}_{p}}^{(-)}$and the "Butler cut-off radius" $R_{0}$ in $M_{l_{n} m_{n}}^{+}$to simulate the nuclear distortions :

$$
\chi_{\vec{q}_{p}^{(-)}(\vec{r})}^{(\vec{\theta})} \chi_{\vec{q}_{d}}^{(\vec{r})}=\theta\left(r-R_{0}\right) e^{i \vec{q}_{p d} \vec{r}}
$$


This leads to

$F_{i_{n}}\left(q_{n} ; \vec{q}_{d}, \vec{q}_{p}\right)=\frac{1}{2} \frac{\mu_{d} \mu_{p}}{\left(\pi \frac{\left.t^{2}\right)^{3}}{t^{3}}\right.} \frac{q_{p}}{q_{d}} q_{n}^{3} m_{n} D_{0}^{2}\left|I_{l_{n}}^{+}\left(q_{p d}, q_{n} ; R_{0}\right)\right|^{2}$,

with

$$
\begin{aligned}
I_{l_{n}}^{+}\left(q_{p d}, q_{n}, R_{0}\right) & =\lim _{\varepsilon \rightarrow 0+} \int_{R_{0}}^{\infty} d r r^{2} e^{-\varepsilon r} j_{l_{n}}\left(q_{p d} r\right) h_{l_{n}}^{(+)}\left(q_{n} r\right) \\
& =\frac{R_{0}^{2}}{q_{p d}^{2}-q_{n}^{2}}\left(q_{p d} h_{l_{n}}^{(+)}\left(q_{n} R_{0}\right) j_{l_{n}}^{\prime}\left(q_{p d} R_{0}\right)-q_{n} h_{l_{n}}^{(+) \prime}\left(q_{n} R_{0}\right) j_{i_{n}}\left(q_{p d} R_{0}\right)\right) .
\end{aligned}
$$

Furthermore, we can write

$$
I_{l n}^{+}=I_{l n}^{j}+i I_{l n}^{n},
$$

where $I_{l_{n}}^{j}$ and $I_{l_{n}}^{n}$ denote the real and imaginary part of $I_{\text {in }}^{+}$(corresponding to a Bessel- and Neumann-function in the integrand).

It is well known from the Butler theory for stripping reactions to bound states that the cut-off radius $R_{0}$ has to be taken larger than the nuclear radius and may be $l_{n}$-dependent. Applying this model to resonances in the reactions ${ }^{15} \mathrm{~N}(\mathrm{~d}, \mathrm{p})^{16} \mathrm{~N}$ (unbound) and ${ }^{24} \mathrm{Mg}(\mathrm{d}, \mathrm{p}){ }^{25} \mathrm{M}$ (unbound) leads - although it is surely very crude to a surprisingly good agreement between theory and experiment, as can be seen in figs. 27 and 28 . In these two figures the influence of the matrix element $M_{i_{n} m_{n}}^{0}$ is also included. Accor- 
ding to the discussion at the beginning of this chapte $r$ this should be most important for low $l_{n}$-values and low neutron energies. Naturally this correction term depends on the background phase shift $\delta_{j n l n}^{(0)}$. For the important case of $\delta_{j n l_{n}}^{(0)} \simeq 0$ the factor $\left|I_{i n}^{+}\right|^{2}$ in eq. (7.14) has to be replaced, in the Butler approximation, where $I_{\ell_{n}}^{j}$ and $I_{l_{n}}^{n}$ are real, by $\left|I_{\ell_{n}}^{n}\right|^{2}-\left|I_{\ell_{n}}^{j}\right|^{2}$.

The experimental data of figs. 27 and 28 were taken from refs. $[119]$ and $[120]$ respectively, where similar fits are given. We note that the correction due to $M_{l_{n} m_{n}}^{0}$ is important only for the $l_{n}=1$ case.

While"parallelism" seems to be the rule, exceptions to it have also been observed (see ref. [35]). We give three possible reasons for this.

First, in the case where the spin of "core $A$ " is different from zero, a mixture of different $j_{n}$ and $l_{n}$ values may contribute to a given resonance. This can easily be seen in the general formula eq. (3.22). There we have $\vec{J}_{B}=\vec{J}_{A}+\vec{j}_{n}$ and $\vec{j}_{n}=\vec{l}_{n}+\frac{1}{2}$ which gives the summation limits of $j_{n}$ and $l_{n}$. Because now the quantity $\sigma_{j n h n}$ is weighted with the stripping enhancement factor according to eq. (7.11), the stripping cross section $d^{2} \sigma / d \Omega_{p} d E_{n}$ may have a different shape as compared to the total elastic scattering cross section $\sigma=\sum_{j n l n} \sigma_{j n} l_{n}$. 
As a second reason, there may be interference with the smooth quantity $M_{l_{n} m_{n}}^{0}$ leading to a change in the resonance shape (called spectator effect in ref. [37]). Again, this special effect can be qualitatively estimated in the framework of the "Butler cut-off approximation", as was done in ref. $[37]$. More generally, we may write, neglecting the part $M_{j m m}^{<} m_{n}$ for the moment : $\frac{d^{2} \sigma_{j n l_{n}}}{d \Omega_{p} d E_{n}} \sim \sum_{m_{n}}\left\{\left|M_{i_{n} m_{n}}^{0}\right|^{2}+2 \operatorname{Pe}\left(M_{l_{n} m_{n}}^{\circ *} O_{j n l_{n}} M_{i_{n} m_{n}}^{+}\right)+\left|O_{j n} l_{n} M_{i_{n} m_{n}}^{+}\right|^{2}\right\}_{(7.17)}$

The first term gives only rise to a structureless background, while the two others vary rapidly with energy. To investigate in more detail the energy behaviour of the resonance line shape we assume that $\delta_{j_{n l n}}$ is real for simplicity's sake and for the moment we decompose $M_{\ln m_{n}}^{+}$- in analogy to eq. (7.16) in *

$$
M_{l_{n} m_{n}}^{+}=M_{l_{n} m_{n}}^{j}+i M_{l n_{n} m_{n}}^{n}
$$

Then we can write

$$
\frac{d^{2} \sigma}{d \Omega_{p} d E_{n}} \sim \sum_{m_{n}}\left|\cos \delta_{j_{n} l_{n}} M_{l_{n} m_{n}}^{j}-\sin \delta_{j_{n} l_{n}} M_{l_{n} m_{n}}^{n}\right|^{2}
$$

From this we get, omitting the structureless background term :

$$
\frac{d^{2} \sigma}{d \Omega_{p} d E_{n}} \sim H_{l_{n}} \sin 2 \delta_{j n l n}+\sin ^{2} \delta_{j n l n}
$$

*) of course, $M_{\text {ln } m m}^{j} \equiv M_{i_{n} m n}^{0}$ 
where

$$
H_{l_{n}}=\frac{\operatorname{Re}\left\{\sum_{m n}\left(M_{l_{n} m_{n}}^{j \times} M_{l_{n} m_{n}}^{n}\right)\right\}}{\sum_{m_{n}}\left\{\left|M_{l_{n} m_{n}}^{j}\right|^{2}-\left|M_{l_{n} m n}^{n}\right|^{2}\right\}} .
$$

The term $H_{\iota_{n}} \sin 2 \delta_{j_{n l n}}$ changes the "on-shell" form $\sin ^{2} \delta_{j_{n} l_{n}}$ of the resonance in a characteristic way. We assume the following one-level Breit-Wigner formula for the resonance phase shift :

$$
\delta_{j n l n}=\delta_{j n l_{n}}^{(0)}+\arctan \frac{\Gamma_{j n l n}}{2\left(E_{R}-E_{n}\right)},
$$

where $\Gamma_{j n l n}$ and $E_{R}$ denote the width and the position of the resonance and the background phase shift is given by $\delta_{j_{n} l_{n}}^{(0)}$. Then we may write :

$$
\begin{aligned}
\frac{d^{2} \sigma_{j n i n}}{d \Omega_{p} d E_{n}} & \sim \frac{1}{1+y^{2}}\left\{y^{2} \sum_{m_{n}}\left|\sin \delta_{j n l_{n}}^{(0)} M_{l_{n} m_{n}}^{n}-\cos \delta_{j n l_{n}}^{(0)} M_{l_{n} m_{n}}^{j}\right|^{2}\right. \\
+2 y \operatorname{Re} & \sum_{m_{n}}\left(\sin \delta_{j n ! n}^{(0)} M_{i_{n} m_{n}}^{n}-\cos \delta_{j n l_{n}}^{(0)} M_{l_{n} m_{n}}^{j}\right)^{*}\left(\sin \delta_{j n ! n}^{(0)} M_{l_{n} m_{n}}^{j}+\cos \delta_{j n l_{n}}^{(0)} M_{l_{n} m_{n}}^{n}\right) \\
& \left.+\sum_{m_{n}}\left|\sin \delta_{j n l_{n}}^{(0)} M_{l_{n} m_{n}}^{j}+\cos \delta_{j n l n}^{(0)} M_{l n m_{n}}^{n}\right|^{2}\right\},
\end{aligned}
$$

with

$$
y=\frac{2\left(E_{n}-E_{R}\right)}{\Gamma_{j n ! n}},
$$

showing in more detail the energy behaviour of the resonance line shape.

It is interesting to compare this energy behaviour with a formula given by Fano. In ref. [121] he treats the interference of a discrete autoionized state with the continuum. This gives rise to characteristically asymmetric peaks in the ex- 
citation spectra of inelastic electron scattering on atoms; they can be parametrized in the following simple form (cf. $\operatorname{ref} \cdot[121$, eq. 21$]$ ) :

$$
\frac{d^{2} \sigma}{d \Omega d E} \sim \frac{(q+y)^{2}}{1+y^{2}}
$$

where $q$ can be regarded as a real constant in the energy region of interest. Generally, this simple form does not hold in continuum stripping but has to be replaced by a slightly more complicated expression. Using Cauchy's inequality, it is easy to show that the energy behaviour of the resonance line shape given by eq. (7.23) can be parametrized as *

$$
\frac{d^{2} \sigma}{d \Omega_{p} d E_{n}} \sim \frac{y^{2}+2|q| \cos \varphi y+|q|^{2}}{1+y^{2}}=\frac{|q+y|^{2}}{1+y^{2}}
$$

i.e. the real quantity $q$ in eq. (7.25) has to be replaced by the complex quantity $q=|q| e^{i \varphi}$, which can be easily found by comparing eq. (7.26) with eq. (7.23). In the special case of the Butler approximation, we have $\varphi=0$, i.e. the Fano formula eq. (7.25) applies.

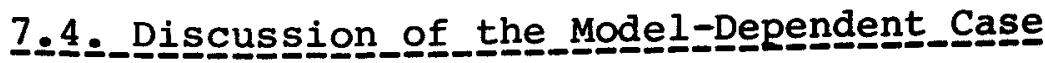

The third reason for the change of the resonance line shape may be that $M_{j n l_{n} m_{n}}^{<}$cannot be neglected. In order to discuss

*) We note that the general form (7.26) can also be achieved in the more complicated "model-dependent case" (see below). 
this term, a good knowledge of the optical model wave functions in the initial and final channel and the neutron resonance wave function is needed. The problem of choosing reliable optical model wave functions is the same as in bound state transfer reactions or in the direct break-up reaction above the coulomb barrier (see above), so we discuss here only the choice of the resonance wave function.

Various different models can be applied to determine wave functions near a resonance. One method would be the real Weinberg formalism, as has been discussed by cole et al. [122] . They compare, in a suitable interaction model, their solution with the exact solution, i.e. that of a coupled channel method. This method will now be described in some detail. The radial wave function $f_{j_{n}(n \lambda}^{J}(r)$ defined in eq. $(3.14) *$ is then explicitly determined by the following system of radial equations [123]

$\left\{\frac{\hbar^{2}}{2 \mu_{n}}\left(\frac{d^{2}}{d r^{2}}-\frac{\ln (\ln +1)}{r^{2}}\right)-V_{j n \ln \lambda}^{J}(r)+E_{n}-\varepsilon_{J}\right\} f_{j n(n)}^{J}(r)$

$$
=\sum_{\left.j n^{\prime} \ln ^{\prime}\right]^{\prime}} V_{j n \ln J \lambda_{j n} \ln ^{\prime} J_{x^{\prime}}^{\prime}}(r) f_{j^{\prime} \ln _{n}^{\prime}}^{J^{\prime}}(r),
$$

where the single particle potentials $V_{j_{n} i_{n}}^{J}(r)$ and the coupling potentials $V_{j n}(n]_{j}, j n^{\prime} \ln ^{\prime} J^{\prime} \lambda^{\prime}(r) \quad$ are determined by the specific

*) Assuming for simplicity's sake that $j_{n}=j_{n}^{\prime}, l_{n}=l_{n}^{\prime}$ and that $f_{j n} l_{n} \lambda$ depends only on $J=J_{A}$. An additional index $\lambda$ is introduced to distinguish different channels with the same angular momentum quantum numbers. 
microscopic model chosen. Let us assume that the resonance is due to a "bound state embedded in the continuum" (BSEC) (cf. ref. [124]). In a simple two-channel model (one closed channel, corresponding to the BSEC $(\lambda=1)$ and one open channel $(\lambda=2))$ the coupled eqs. (7.27) can be conveniently written in matrix-form:

$$
\frac{\hbar^{2}}{2 \mu_{n}}\left(\frac{d^{2}}{d r^{2}}-\frac{\ln (\ln +1)}{r^{2}}\right)\left(\begin{array}{l}
f_{1} \\
f_{2}
\end{array}\right)=\left(\begin{array}{cc}
V_{1}(r)-\varepsilon+E_{0}-E_{n} & V_{12}(r) \\
V_{21}(r) & V_{2}(r)-E_{n}
\end{array}\right)\left(\begin{array}{l}
f_{1} \\
f_{2}
\end{array}\right),
$$

where later on $f_{2}(r)$ has to be identified with the radial wave function $f_{j n l n}^{J}$ - The energy $E_{0}$ is a constant chosen essentially to shift the resonance to the desired position and $\varepsilon$ is a bound state eigenvalue in the potential $V_{1}(r)$. Since the specific form in which the BSEC is coupled to the continuum is in general poorly known, we investigate two extreme cases for such a coupling. This will give us a feeling of the freedom which one has in the choice of the resunance wave function. So we assume real square well potentials for $V_{i}(r)$ :

$$
V_{i}(r)=-\theta\left(R_{0}-r\right) \bar{V}_{i}, \quad i=1,2 .
$$

For the coupling potential we take an extreme "surface" type of coupling ("model SC"), defined by

$$
V_{12}(r)=V_{21}(r)=C_{1} \cdot \delta\left(r-R_{0}\right)
$$

and, on the other hand, a "volume" type of coupling ("model VC"), i.e.

$$
V_{12}(r)=V_{21}(r)=C_{2} \theta\left(R_{0}-r\right)
$$

It is possible to solve these coupled channel models in closed form. The details are worked out in refs. $[125,126]$. In the 
case of "model SC" the result can be described in very simple terms. While the s-matrix elements for the asymptotic part of $f_{j n} l_{n}$ are easily found by the matching condition at $r=$ Ro, the resonance wave function for $r<$ Ro can be factorized to a very good approximation in the following way :

$$
f_{j n l n}\left(E_{n}, r\right)=a\left(E_{n}\right) v_{j n} l_{n}(r)
$$

The energy dependence of the resonance wave function is determined by

$$
a\left(E_{n}\right)=\frac{\Gamma_{i n} e^{i \delta_{j n} l_{n}}}{\sqrt{P_{l n}\left(4\left(E_{n}-E_{R}\right)^{2}+\Gamma_{j n}^{2} l_{n}\right)}},
$$

where $P_{l_{n}}$ is the usual penetrability factor, and the shape of the wave function is essentially given by

$$
v_{j n l_{n}}(r)=\frac{j_{l_{n}}\left(k_{n} r\right)}{j_{l_{n}}\left(k_{n} R_{0}\right)} \text {. }
$$

Here $k_{n}$ is the wave number in the nuclear interior, eq. (4.50), which is approximately constant over the resonance energy region. We can see that the wave function becomes largest for $E_{n}=E_{R}$, therefore, the contribution of $M_{j n l_{n} m_{n}}^{<}$tends to make the resonance line shape symmetric even if the background phase shift $\delta_{j n i n}^{(0)}$ is different from zero.

As an example we consider again the reaction ${ }^{15} \mathrm{~N}(\mathrm{~d}, \mathrm{p}){ }^{16} \mathrm{~N}$ (unbound) which was measured at $\mathrm{E}_{\alpha}=12 \mathrm{MeV}$ by Fuchs et al. $[34,35]$. We are especially interested in the behaviour of the resonance 
line shape at $E_{n}=3.454 \mathrm{MeV}$. The corresponding elastic scattering shows a resonance line shape which corresponds to a background phase shift of $\delta_{\iota_{n}} \simeq-\frac{\pi}{4}$. This asymmetric line shape becomes symmetric in the $(d, p)$-spectrum if the stripping angle is increased from $\theta_{p}=7.5^{\circ}$ to $\theta_{p}=30^{\circ}$. This can be seen in fig. 29, where also a comparison with our model calculations ("model SC") is given. The difference between the different model calculations can be seen in fig.30, where "model SC" is compared with "model VC". Note that both models give rise to the same phase shifts. As a third curve, the "cut-off model" is shown, where the contributions arising from the term $M_{j n l_{n} m_{n}}^{<}$are entirely neglected. This example may serve to show what kinds of conclusions can be drawn from the study of resonance line shapes. However, it should be noted, that for a careful analysis the zero-range approximation used here, should be refined in order to take finiterange effects into account. This can of course be done, e.g. in the manner discussed in chapters 4.2 and 4.3.

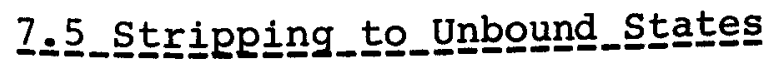

We define the cross section of stripping to an unbound state by an integral of $d^{2} \delta / d \Omega_{p} d E_{n}$ over the energy interval of the resonance considered. The smooth background should be subtracted. This is schematically illustrated in fig. 31. Here we assume that we deal with non-overlapping resonances. (For the case of overlapping resonances we refer the reader to the papers of Vincent and Fortune [116] and Hüfner and Sevgen $[127]$ ). Again,we assume the Breit-Wigner resonance 
formula (eq. 7.22). The "model-independent case" becomes simple, since the phase $\delta_{j_{n} \text { ln }}\left(E_{n}\right)$ is the only rapidly varying function in the energy interval over the resonance. Therefore, we can easily carry out the integration over the energy. For the resonant partial wave we have

$$
\begin{aligned}
& \frac{d \sigma_{j n} l_{n}}{d \Omega_{p}} \equiv \int d E_{n}\left(\frac{d^{2} \sigma_{j n l n}}{d \Omega_{p} d E_{n}}-\frac{d^{2} \sigma_{j n l n} \text { (non-resonant) }}{d \Omega_{p} d E_{n}}\right) \\
& \simeq \frac{1}{2} \cos 2 \delta_{j n l_{n}}^{(0)} \cdot \frac{\mu d \mu_{p}}{\left(2 \pi t^{2}\right)^{2}} \cdot \frac{q_{n} m_{n} \Gamma_{n n l_{n}}}{\hbar^{2}} \frac{q_{p}}{q_{d}} \frac{2 J_{B}+1}{2 J_{n}+1} \frac{D_{0}^{2}}{2 l_{n}+1} \\
& \\
& \cdot \sum_{m_{n}}\left|M_{l_{n} m_{n}}^{+}\right|^{2},
\end{aligned}
$$

where the smooth part due to the background phase shift has also been substracted. Hereby we have used that

$$
\begin{aligned}
\int_{E_{R}-\Delta_{E}}^{E_{R}+\Delta_{E}} d E_{n}\left(\sin ^{2} \delta_{j n}-\sin ^{2} \delta_{j n ! n}^{(0)}\right) & \simeq \int_{-\infty}^{+\infty} d E_{n}\left(\sin ^{2} \delta_{j n l n}-\sin ^{2} \delta_{j n l n}^{(0)}\right) \\
& =\frac{\pi}{2} \Gamma_{j n l n} \cdot \cos 2 \delta_{j n l n}^{(0)},
\end{aligned}
$$

where we have assumed that the width $\Gamma_{j n}$ is much smaller than the integration interval $2 \Delta_{E}^{*}$. Note that $d \sigma_{\text {min }} /$ lep $_{p}$ defined in eq. (7.35) can also become negative, e.g. in the case of a background phase shift $\delta_{j_{n} l_{n}}^{(0)}=\frac{\pi}{2}$, where the resonance manifests itself as a dip in the spectrum. Of course, at the point where $\delta_{j n} l n$ is zero, some correction due to the non-vanishing contribution of $M_{l_{n} m_{n}}^{0}$ should be applied. This can easily be done in the manner described above.

*) Of course, it is very easy to generalize eq. $(7.36)$ to the case, where $\Gamma_{l_{n} l_{n}} \simeq \Delta_{E}$. 
In stripping reactions to unbound states, described by eq. (7.35), it is in principle not necessary to introduce the concept of a spectroscopic factor. However, in order to have a convenient measure of the "single particle strength" of an unbound state, a spectroscopic factor $\varphi$ may be defined by $[128] \varphi=\Gamma_{\text {jnln }} / \Gamma_{w} \quad$, where $\Gamma_{w}$ denotes the single particle width which is defined by $\Gamma_{w}=2 P_{n} \gamma_{w}^{2}$ and $\gamma_{w}^{2}$ is the Wigner limit.

Although eq. (7.35) can be calculated by existing DWBA-codes and using the integration technique of Vincent and Fortune, a semiclassical treatment of the reaction may again lead to a deeper physical insight. In the sub-Coulomb energy region eq. (6.10) leads immediately to :

$$
\begin{gathered}
\frac{d \sigma_{j n i n}}{d \Omega_{p}}=2 \pi \cos 2 \delta_{j n ! n}^{(0)} \cdot\left(\frac{2}{\hbar^{2}}\right)^{3} \frac{\mu_{d} \mu_{p} m_{n}}{Q^{2} q_{n}} \frac{q_{p}}{q_{d}}\left|\bar{f}_{c}\right|^{2}\left|A_{0}\right|^{2} \frac{2 J_{B+1}}{2 J_{n+1}} \Gamma_{j n ! n} . \\
\cdot \sum_{m_{n}}\left|Y_{l_{n} m_{n}}\left(\frac{\pi}{2}, 0\right) I_{l_{n} m_{n}}\left(\varepsilon, \xi, \xi^{\prime}, \rho\right)\right|^{2} .
\end{gathered}
$$

Due to our sub-Coulomb condition we have replaced the zerorange constant $D_{0}^{2}$ by the form factor $A_{0}^{2}\left(q_{n}\right) / 4 \pi$. The simple semiclassical expression (7.37) shows the great similarity between stripping reactions to unbound states and to bound states. All the kinematical relations valid in the latter case are also valid in stripping reactions to unbound states [110]. As an example, we have given in fig. 30 some model calculations for the reaction ${ }^{208} \mathrm{~Pb}(\mathrm{~d}, \mathrm{p}){ }^{209} \mathrm{~Pb}$ (unbound) to show the strong $Q$-value dependence well known from bound stripping $[129]$. In this case we have chosen $l_{n}=2$ and 
$F_{n} l_{n}=1 \mathrm{keV}$. More important is the above mentioned $l_{n}$-enhancement in unbound stripping reactions which can be seen directly in figs. 33 and 34 . There we have chosen $\Gamma_{\text {jnln }}=$ constant $=1 \mathrm{keV}$ to show this enhancement more clearly. We emphasize that this choice means that the corresponding reduced widths $\gamma_{j n l_{n}}^{2}$ depend on $l_{n}$ according to the relation $\Gamma_{j n l_{n}}=2 P_{n} \gamma_{j n l_{n}}^{2}$. The neutron penetrability $P_{i_{n}}$ behaves like $\left(q_{n} R_{n}\right)^{2 l_{n}+1 /} /\left(2 l_{n}-1\right) !$ ! for low neutron energies and since this factor is approximately cancelled by the term originating from the Hankel function, all $l_{n}$-values are weighted similarly. This is not so for neutron scattering and therefore stripping reactions to unbound states should be well suited for the study of resonances with large $l_{n}$-values.

of course, at higher energies of the deuteron again "modeldependent" contributions from the nuclear interior will show up. As an example we have calculated the reaction ${ }^{208} \mathrm{~Pb}(\mathrm{~d}, \mathrm{p})$ ${ }^{209} \mathrm{~Pb}$ (unbound) at $\mathrm{E}_{d}=12 \mathrm{MeV}$ and have made a comparison with the experimental results of Ellegard et al. $[130]$. The optical potentials chosen are again those of ref。 [81]. For the description of the resonance wave function we choose a BSEC with the two extreme coupling potentials described above (for further details see ref. $[125]$ ). As can be seen in fig. 35, some small effects of the nuclear interior show up but they are rather insensitive to the specific coupling potentials. (Note in this context that in the direct break-up process at this energy the nuclear interior can safely be neglected since it is not enhanced by a resonance behaviour of the wave functions). The shape of the angular distribution turns 
out to be quite insensitive to the $j_{n}-$ and the $l_{n}-$ value of the resonance, the value of $\Gamma_{j_{n}} l_{n}$ determines the absolute value of the cross section. In fig. 35 we have chosen $j_{n}=\frac{g}{2}, l_{n}=4$ and $\Gamma_{j n l_{n}}=11 \mathrm{ev}$. Going to energies higher than $12 \mathrm{MeV}$ the contributions from the interior will become larger, allowing possibly some more specific statements about the resonance wave function.

8. Comparison and Connection of Various Theories on Continuum stripping

Numerous papers have appeared on continuum stripping during the last few years. All these papers may be conveniently divided into two categories :

(i) The unbound state is treated as a continuum scattering state. (ii) Some kind of "wave function for an unbound state" is defined which has properties similar to a bound state wave function. This wave function is then usually introduced into the DWBA matrix element.

Theories of category (ii) may be more directly related to the corresponding bound state stripping; they are, however, special cases in the more general type of theories in category (i), which we will mainly discuss here. Before doing this, let us briefly describe some work of group (ii) which shows very interesting mathematical aspects. Zel'dovich $[131]$ showed how to introduce a suitable normalization of an exponentially decaying state described by a wave function $\chi_{n}$ by performing 
the following limit

$$
\lim _{\varepsilon \rightarrow 0^{+}} \int d r r^{2} \chi_{n}^{2} e^{-\varepsilon r^{2}} \text {. }
$$

This idea has been used by Berggren [132] who has shown that norms can be introduced for Gamov states such that many analoguous mathematical properties are shared by bound states and complex-energy eigenstates. This method was applied by Bang and zymanyi [43] to stripping to unbound states. They treat the reaction ${ }^{208} \mathrm{~Pb}(\mathrm{~d}, \mathrm{p}){ }^{209} \mathrm{~Pb}$ (unbound) as an example. This approach was further pursued by coker $[44]$ who analyzed the reaction ${ }^{36,38} \mathrm{Ar}(\mathrm{d}, \mathrm{p})$ leading to neutron resonances in $37,39 \mathrm{Ar}$.

Another approach was described by Schlessinger and Payne [49] who use a resonant wave function which was introduced by Coester and schlessinger $[133]$. This function is square integrable and is an exponentially decreasing function of $r$ outside the range of the potential. Thus, this method may be used in existing DWBA codes simply by replacing the bound state wave functionby a wave function which has to be constructed in a somewhat unconventional way. The authors calculate the cross section for the ${ }^{16} \mathrm{O}(\mathrm{d}, \mathrm{p}){ }^{17} \mathrm{O}$ (unbound) transfer reaction to the resonant $5.08 \mathrm{MeV}$ state of ${ }^{17} \mathrm{O}$. Their results agree to order $\Gamma / E_{R}$ with other methods.

Practically all the work in category (i) is based on the postform of the DWBA originally given by Huby and Mines [38]. Working directly in configuration space has become convenient 
since the introduction of the complex integration method by Vincent and Fortune [42] . Thus, all these approaches are similar in spirit, they only differ in their emphasis of certain properties of the wave function of the transferred particle.

Cole, Huby and Liu [122] calculate the contributions from the interior region; they introduce the Weinberg method to construct the wave function for the resonant state, while Barz et al. [123] use the coupled channel formalism. In principle, all kinds of microscopic reaction theories could be used to compute the wave function of the resonant state. The drawback of such an approach could be the model dependence and, from a practical point of view, the fact that most of the resonances occurring in real nuclei are too complicated to be described in that way (for example, $\alpha$-unstable states would require a theoretical treatment where 4 nucleons are in the continuum).

The introduction of the "on-shell" or "peripheral" approximation $[28,37,116,118]$ simplifies the whole problem considerably and makes it model independent. The only "input" is the phase shift of the elastic scattering of the transferred particle on the target nucleus. Furthermore, the most important features are reproduced. Of course, this method would fall if the contributions from the interior are very important. However, in the particularly "clean" situations of sub-Coulomb or heavy iontransfer the conditions for this approximation are very well fulfilled. The "peripheral" approximation has also been used 
implicitly by Sharaf $[54]$ who constructs a diffraction model and obtains analytical expressions for the distorted wave integrals. He obtains good agreement with experimental data for the reaction ${ }^{92} \mathrm{Mo}\left({ }^{3} \mathrm{He}, \mathrm{d}\right)^{93} \mathrm{TC}$ leading to various unbound states. The agreement is not quite as good for the ${ }^{16} \mathrm{o}(\mathrm{d}, \mathrm{p}){ }^{17} \mathrm{O}_{5,08 \mathrm{MeV}}$ reaction, probably, according to the author, because the deuteron or especially the proton are not strongly absorbing particles.

Another approach is considered by Bunakov $[45-48]$ who used wave functions of the $(A+n)$ state for real energies and adopted Schrödinger's equation to transform to the prior form the part of the integral in which the particle $n$ is beyond the nuclear force range. As a result of this transformation a surface term arises. This term is considered in Bunakov's treatment as giving the dominant contribution.

In an attempt to circumvent the convergence problem of the post-form DWBA, Noble [53] advocated a momentum space approach. The distorted waves are written in momentum space in terms of the off-shell two body scattering amplitudes in the following way

$$
\Psi_{\vec{k}}^{ \pm}\left(\vec{k}^{\prime \prime}\right)=\delta\left(\vec{k}-\vec{k}^{\prime \prime}\right)+\frac{\left\langle\vec{k}^{\prime \prime}\left|T\left(E_{k}+i \varepsilon\right)\right| \vec{k}\right\rangle}{E_{k} \pm i \varepsilon-E_{k^{\prime \prime}}}
$$

Thus the usual zero-range DWBA expression can be rewritten as $T_{f i}^{(t)} \sim \iint d^{3} k_{1} d^{3} k_{2} \Psi_{\vec{q}_{p}}^{(-) *}\left(\vec{k}_{1}\right) \Psi_{\vec{q}_{n}}^{(-) *}\left(\vec{k}_{2}\right) \Psi_{\vec{q}_{d}}^{(+)}\left(\vec{k}_{1}+\vec{k}_{2}\right)$. 
Inserting (8.2) one obtains seven terms (ignoring the vanishing $\delta$-function term) which contain no divergent integrals but merely Cauchy singularities, which are straightforward to handle numerically, according to the author. However, this method has so far not been applied in practice. It seems to be more cumbersome than a direct evaluation of the matrix element in $r$-space, especially when the Vincent and Fortune integration method is used. An approach similar in some respect to the one in ref. [53] was given by Lipperheide [50] who started directly from a graph technique*. In the plane-wave Born approximation, a relation of the stripping cross section to the imaginary part of the off-energy shell forward amplitude for elastic scattering on the target nucleus is established, using the off-energy-shell optical theorem. This clarifies in a nice way the relation between transfer into the continuum and elastic scattering, and is intuitively very appealing. However, it is not clear whether these off-shell amplitudes can really be extracted unambiguously from a comparison with the experimental data. A method to calculate these off-shell matrix elements in the frame of R-matrix theory has been given in refs. $[51,52]$. E.g. in the plane wave limit given by

$$
\Psi_{\vec{q}_{p}}^{(-)}\left(\vec{k}_{p}^{\prime}\right)=\delta\left(\vec{k}_{p}^{\prime}-\vec{q}_{p}\right) \quad \text { and } \quad \Psi_{\vec{q}_{d}}^{(+)}\left(\vec{k}_{d}^{\prime}\right)=\delta\left(\vec{q}_{d}-\vec{k}_{d}^{\prime}\right),
$$

the cross section can be expressed in terms of the "half

*) The graph technique has also been used by Dolinsky and coworkers [55-57] in their peripheral treatment of stripping reactions to unbound states. 
off-shell" matrix element defined by $\left\langle\vec{q}_{\alpha}-\vec{q}_{p}\left|T\left(E_{n}\right)\right| \vec{q}_{n}\right\rangle$

as can be directly seen from eqs. (8.2) and (8.3). However, switching on the distortion makes this simple relation invalid.

9. Conclusion and Outlook

We have presented here essentially the DWBA expression for the break-up of the deuteron in the post form. A comparison of this theory with experiment, especially in the sub-Coulomb energy region, where the test is most meaningful, shows very good agreement. This gives us confidence in extending the theory to stripping reactions to unbound states. Features like the "ln-enhancement" and the "parallelism" are now well established; the observation of resonance line shapes is a potential tool for the investigation of resonance wave functions. This will necessitate more detailed experimental and theoretical studies.

Especially interesting is the application of the general formalism to projectiles other than the deuteron $[134]$. As far as neutron transfer is concerned, it is necessary to have projectiles with low neutron binding energy because of Q-value requirements.. ${ }^{9}$ Be will therefore be a favourable candiate. However, in general, the kinematic effects are expected to be the same as in deuteron induced reactions. Charged particle transfer reactions, on the other hand, often have negative 
"optimal Q-values" [129], which favours transfer to unbound states $[135,136]$. The theoretical description of these processes is important and can be given in this framework. Naturally, the transferred particle should be transferred "as a lump", whereas many additional complications will arise if unstable systems, like two protons, are transferred. 


\section{References}

1. J.R. Oppenheimer and M.Phillips, Phys.Rev.48 (1935) 500

2. J.R. Oppenheimer, Phys.Rev. 47 (1935) 845

3. S.T.Butler, Proc.Roy.Soc. (London) 208 (1951) 559

4. N.Austern, Direct Nuclear Reaction Theory (Wiley, New York 1970)

5. F.P.Gibson and A.K.Kerman, Phys.Rev. 145 (1966) 758

6. R.C.Johnson and P.J.Soper, Phys.Rev.Cl (1970) 976

7. L.Landau and E.Lifshitz, JETP 18 (1948) 750 (English translation in Collected Papers of L.Landau)

8. G.Breit in Handbuch der Physik, ed. by S.Flügge (SpringerVerlag Berlin) Vol. 41, 304-320

9. W.B.Ketchum, M.D.thesis, University of Pittsburgh (1960) unpublished

10. G.Baur and D.Trautmann, Nucl.Phys.Al91 (1972) 321

11. N.Austern, private communication

12. C.J.Mullin and F.Guth, Phys.Rev. 82 (1951) 141

13. R.Gold and C.Wong, Phys.Rev. 132 (1963) 2586

14. B.L.Cohen and C.E.Falk, Phys.Rev. 84 (1951) 173

15. F.A.Aschenbrenner, Phys.Rev. 98 (1955) 657

16. E.w.Hamburger, B.L.Cohen and R.E.Price, Phys.Rev. 121 (1961) 1143

17. E.C.May, B.L.Cohen and T.M.O'Keefe, Phys.Rev. 164 (1967) 1253

18. C.I.Fink, B.L.Cohen, J.C.van der Weerd and R.J.Petty, Phys.Rev. 185 (1969) 1568

19. F.Udo, H.R.E.Tjin A Djie and L.A.Ch.Koerts, Nucl.Phys.63 (1965) 657

20. F.Udo and L.A.Koerts, Nucl.Phys.70 (1965) 145

21. F.Udo, Rev.Mod.Phys. 37 (1965) 365

22. O.F.Nemets, M.V.Sokolov and B.G.Struzhko, Sov.J.Nucl.Phys.1 (1965) 722

23. A.Pollitzer, W.Wölfli, J.Lang,R.Müller and P.Marmier, Helv.Phys.Acta 41 (1968) 439

24. L.Jarczyk, J.Lang, R.Müller, A.Pollitzer, J.F.Valley, W.Wölfli and P.Marmier, Phys.Lett. 39B (1972) 191

25. J.Lang, L.Jarczyk and R.Müller, Nucl.Phys. A204 (1973) 97

26. L.Jarczyk, J.Lang, R.Müller, D.Balzer, P.Viatte and P.Marmier, Phys.Rev. C8 (1973) 68

27. F.Rybicky and N.Austern, Phys.Rev. C6 (1972) 1525 
28. G.Baur and D.Trautmann, Nucl.Phys. Al99 (1973) 218

29. G.Bau $r$ and D.Trautmann, Phys.Lett. 42B (1972) 31

30. G.Baur, D.Trautmann and V.zoran, Nucl.Phys. A208 (1973) 261

31. G.Baur and D.Trautmann, Nucl.Phys. A211 (1973) 333

32. G.Baur and D.Trautmann, J.Appl.Math. and Physics (ZAMP) 25 (1974) 9

33. J.I.Alty, L.L.Green, R.Huby, G.D.Jones, J.R.Mines and J.F.Sharpey-Schafer, Nucl.Phys. A97 (1967) 541

34. H.Fuchs, H.Homeyer, Th.Lorenz and H.Oeschler, Phys.Lett. 37B (1971) 285

35. H.Fuchs, H.Homeyer, H.Oeschler, R.Lipperheide and K.Möhring, Nucl.Phys. Al96 (1972) 286

36. F.L.Friedmann and W.Tobocman, Phys.Rev. 92 (1953) 93

37. G.Baur and D.Trautmann, Z.Physik 267 (1974) 103

38. R.Huby and J.R.Mines, Rev.Mod.Phys. 37 (1965) 406

39. C.M.Vincent, Phys.Rev. 175 (1968) 1309

40. F.S.Levin, Ann.Phys. 46 (1968) 41

41. F.S.Levin, Nucl.Phys. Al15 (1968) 449

42. C.M.Vincent and H.T.Fortune, Phys.Rev. C2 (1970) 782

43. J.Bang and J.Zimany, Nucl.Phys. Al39 (1969) 534

44. W.R.Coker, Phys.Rev. C7 (1973) 2426

45. V.E.Bunakov, Nucl.Phys. Al40 (1970) 241

46. V.E.Bunakov, K.A.Gridnev and L.V.Krasnov, Phys.Lett. 32B (1970) 587

47. V.E.Bunakov, K.A.Gridnev and L.V.Krasnov, Sov.J.Nucl.Phys. 15 (1972) 508

48. V.E.Bunakov, K.A.Gridnev and L.V.Krasnov, Phys.Lett. 34B (1971) 27

49. L.Schlessinger and G.L.Payne, Phys.Rev. C6 (1972) 2047

50. R.Lipperheide, Phys.Lett. 32B (1970) 555

51. R.Lipperheide and K.Möhring, Nucl.Phys.A211 (1973) 125

52. K.Möhring and R.Lipperheide, Nucl.Phys. A21l (1973) 136

53. J.V.Noble, Phys.Rev. Cl (1970) 1742

54. M.A.Sharaf, Z.Physik 253 (1972) 28

55. P.O.Dzhamalov and E.I.Dolinsky, Sov.J.Nucl.Phys. 14 (1972) 423

56. P.O.Dzhamalov, E.I.Dolinsky and A.M.Mukhamedzhanov, Sov.J. Nucl.Phys. 15 (1972) 147

57. E.I.Dolinsky, P.O.Dzhamalov and A.M.Mukhamedzhanov, Nucl.Phys. A202 (1973) 97 
58. S.E.Darden, S.Sen, H.R.Hiddleston, J.A.Aymar and W.A.Yoh, Nucl.Phys. 208 (1973) 77

59. M.D.Cooper, W.F.Hornyak and P.G.Roos, Nucil.Phys. A218 (1974) 249 60. S.Sen, S.E.Darden, H.R.Hiddleston and W.A.Yoh, Nucl.Phys.
A219 (1974) 429

61. R.G.Newton, Scattering Theory of Waves and Particles, (McGraw-Hill, New York 1966)

62. G.G.Ohlsen,Nucl.Instr.Meth. 37 (1965) 240

63. B.L.Gambhir and J.J.Griffin, Phys.Rev. C5 (1972) 1856

64. B.L.Gambhir and J.J.Griffin, Phys.Rev. C7 (1973) 590

65. B.L.Gambhir and J.J.Griffin, Phys.Lett. 50B (1974) 407

66. N.Austern, Phys.Lett. 46B (1973) 49

67. E.Gerjuoy, Ann.Phys. 5 (1958) 58

68. K.R.Greider and L.R.Dodd, Phys.Rev. 146 (1966) 671

69. W.A.Friedman, Phys.Rev。C6 (1972) 87

70. L.Hulthèn and M.Sugawara, in Handbuch der Physik, ed. by S.Flügge (Springer-Verlang, Berlin 1957), Vol. 39, Sec. 33

71. G.R.Satchler, Nucl.Phys. 55 (1964) 1

72. P.J.A.Buttle and L.J.B.Goldfarb, Nucl.Phys. 78 (1966) 409

73. T.Sawaguri and W.Tobocman, J.Math.Phys. 8 (1967) 2223

74. K.Alder, A.Bohr, T.Huss, B.Mottelson and A.Winther, Rev. Mod.Phys. 28 (1956) 432

75. A.Sommerfeld, Atombau und Spektrallinien, Bd.2 (Vieweg, Braunschweig 1939)

76. A.Nordsieck, Phys.Rev. 93 (1954) 785

77. M.Abramowitz and I.A.Stegun, Handbook of Mathematical Functions (Natl.Bur.Stds, Washington D.C.1964)

78. W.E.Frahn and R.H.Venter, Ann.Phys. 24 (1963) 243

79. K.Alder and D.Trautmann, Ann.Phys. 66 (1971) 884

80. C.M.Perey and F.G.Perey, Phys.Rev。 132 (1963) 755

81. D.Wilmore and P.E.Hodgson, Nucl.Phys. 55 (1964) 673

82. G.Baur, F.Rösel and D.Trautmann, to be published

83. P.J.A.Buttle and I.J.B.Goldfarb, Proc.Phys.Soc.83 (1964) 701

84. A.Dar, A.de Shalit and A.S.Reiner, Phys.Rev. 131 (1963) 1732

85. M.El Nadi and T.H.Rihan, Proc.Phys.Soc. 87 (1966) 119

86. R.Anni and L.Taffara, RivoNuovo Cimento 2 (1970) 1

87. H.Feshbach, C.Porter and V.F.Weisskopf, Phys.Rev. 96 (1954) 448

88. M.Danos and L.C.Maximon, J.Math.Phys. 6 (1965) 766 
89. K.A.Ter-Martirosyan, JETP (Sov.Phys.) 2 (1956) 620

90. A.Messiah, Quantum Mechanics (North-Holland, Amsterdam 1970)

91. K.M.Watson, Phys.Rev. 88 (1952) 1163

92. A.B.Migdal, JETP (Sov.Phys.) 1 (1955) 2

93. P.Appell and M.J.Kampé de Fériet, Fonctions hypergéométriques et hypersphériques (Gauthier-Villars, Paris 1926)

94. D.Trautmann and K.Alder, Helv.Phys.Acta 43 (1970) 363

95. P.O.M.Olsson, Arkiv för Fysik 29 (1965) 459

96. P.O.M.Olsson, ibid. 30 (1965) 187

97. F.D.Bechetti, Jr. and G.W.Greenlees, Phys.Rev. 182 (1969) 1190

98. J.A.M.de Villiers, C.A.Engelbrecht, W.G.Vonach and A.B.Smith, Z.Physik 183 (1965) 323

99. T.A.Griffy, Phys.Lett. 21 (1966) 693

100. R.J.Drachmann, Phys.Rev.Lett. 17 (1966) 1017

101. N.F.Ramsey, B.J.Malenka and U.E.Kruse, Phys.Rev. 91 (1953) 1162

102. B.W.Downs, Phys.Rev. 98 (1955) 194

103. G.Bencze and J.zimany, Phys.Lett. 9 (1964) 246

104. P.Braun-Munzinger and H.L.Harney, Nucl.Phys. A223 (1974) 381

105. R.C.Johnson, Nucl.Phys. A90 (1967) 289

106. R.C.Johnson and F.D.Santos, Particles and Nuclei, 2 (1971) 285

107. W.Hauser and H.Feshbach, Phys.Rev. 87 (1952) 366

108. L.Wolfenstein, Phys.Rev. 82 (1951) 690

109. P.A.Moldauer, Phys.Rev. 123 (1961) 968

110. K.Alder, R.Morf, M.Pauli and D.Trautmann, Nucl.Phys. Al9l (1972) 399

111. N.F.Mott and H.S.W.Massey, The Theory of Atomic Collisions (Clarendon Press, Oxford 1949)

112. R.Broglia and A. Winthe r, Phys.Lett C4 (1972) 153

113. G.Baur, M.Pauli and D.Trautmann, Nucl.Phys. A224 (1974) 477

114. G.Breit and P.B.Daitch, Proc.Nat.Acad.Sci. USA 41 (1955) 653

115. E.P.Wigner, Phys.Rev. 98 (1955) 145

116. C.M.Vincent and A.T.Fortune, Phys.Rev. C8 (1973) 1084

117. B.J.Cole and R.Huby, Phys.Lett. 46B (1973) 55

118. R.Huby and D.Kelvin, J.Phys. Gl (1975) 203

119. K.Möhring, R.Lipperheide and B.J.Cole, Nucl.Phys. A243 (1975) 365 
120. J.Bommer, H.Fuchs, K.Grabisch, R.Lipperheide, K.Möhring and H.Oeschler, Proc.Int.Conf.on Nucl.Phys.,Munich (1973), Vol.I, pg 503

121. U.Fano, Phys.Rev. 124 (1961) 1866

122. B.J.Cole, R.Huby and O.K.K.Liu, J.Phys. A6 (1973) 1224

123. H.W.Barz, V.E.Bukanov and A.M.E1-Naiem, Nucl.Phys. A217 (1973) 141

124. C.Mahaux and H.A.Weidenmüller, Shell-Model Approach to Nuclear Reactions (North-Holland, Amsterdam, 1969)

125. G.Baur, F.Rösel and D.Trautmann, Nucl.Phys. A252 (1975) 77

126. R.G.Lovas, Acta Physica Academiae Sc.Hung. 36 (1974) 165

127. J.Hüfner and A.Sevgen, Nucl.Phys. A 218 (1974) 239

128. J.P.Schiffer, Nucl.Phys. 46 (1963) 246

129. P.J.A.Buttle and L.J.B.Goldfarb, Nucl.Phys.A176 (1971) 299

130. C.Ellegard, J.Kantele and P.Vedelsby, Nucl.Phys. Al29 (1969) 113

131. Y.A.B.Zel'dovich, JETP (Sov.Phys.) 12 (1961) 542

132. T.Berggren, Nucl.Phys. Al09 (1968) 265

133. F.Coester and L.Schlessinger, Ann.Phys. 78 (1973) 90

134. G.Baur, M.Pauli and D.Trautmann, to be published

135. L.Ray, W.R.Coker and T.Udagawa, Phys.Lett. 56B (1975) 318

136. L.R.Medsker, H.T.Fortune and S.C.Headley, Phys.Rev. C12 (1975) 401 
Figure Captions

Fig.-1

The system of coordinate vectors.

Fig._는

Comparison of a full DWBA calculation for the deuteron break-up on ${ }^{208} \mathrm{~Pb}$ at $\mathrm{Ed}=17 \mathrm{MeV}$ and $\mathrm{Ep}=11 \mathrm{MeV}$ with the experimental results of ref. [18]. The cross section is shown as a function of the neutron angle $\theta_{n}$ (for fixed $\theta_{p}=40^{\circ}$ ). The effect of finite-range is shown separately.

\section{Fig. -3}

The function $G(R)$ eq. $(4.37)$ is shown for the break-up reaction on $\mathrm{Au}$ for $\mathrm{Ed}=12 \mathrm{MeV}, \mathrm{Ep}=8 \mathrm{MeV}$ and $\theta_{n}=20^{\circ}$ for different proton angles. (Note the scale :).

Fig. $--\underline{4}$

Same as in fig. 3 but for $\mathrm{Ed}=18 \mathrm{MeV}$ and $\mathrm{Ep}=13 \mathrm{MeV}$.

\section{Fig. $-\underline{5}$}

The exact and approximate radial form factors $Z_{j u l_{n}}^{L l_{2}}(r)$ eqs. (4.51, 4.52) are shown for $E_{n}=0.5 \mathrm{MeV}$ and $L=l_{2}=l_{n}=0$. The real and imaginary parts are given separately. 


\section{Fig $\underline{\text {. }}-\underline{6}$}

Comparison of the exact and the semiclassical radial integrals (cf. eq.(4.91)). The real and imaginary parts are given separately.

\section{Fig. -7}

Energy dependence of the break-up reaction on $\mathrm{Au}$. The differential cross section $d^{2} \sigma / d \Omega_{p} d \Omega_{n}$ for two neutronproton angle combinations is shown as a function of the deuteron energy. In dashed lines, the predictions for pure Coulomb break-up are given, and in the dot-dashed curve the $n$ - Au interaction is included. The solid line includes also the nuclear deuteron-target and proton-targetractionexperimental points are taken from ref. $[26]$.

\section{Fig._- 8}

Proton-neutron angular correlation for the break-up reaction on $\mathrm{Au}$ at $\mathrm{E}_{d}=10.5 \mathrm{MeV}$ as a function of the proton angle $\theta_{p}$ (for fixed $\theta_{n}=40^{\circ}$ ).

\section{Fig. -9}

Comparison of our calculation for the deuteron break-up coincidence cross section on $\mathrm{Au}$ at $\mathrm{E}_{d}=12 \mathrm{MeV}$ with the experimental results of ref. [24]. A coplanar geometry was used and the neutron and proton angles are $\theta_{n}=20^{\circ}$ and $\theta_{p}=50^{\circ}$.

\section{Fig._1무}

The same as in fig. 9 but for $\theta_{n}=20^{\circ}$ and $\theta_{p}=-60^{\circ}$. 


\section{Fíg._-1노}

The dependence of the break-up cross section on the optical model parameters for the neutron-target interaction.

\section{Fig. $-\underline{12}$}

The deuteron break-up on ${ }^{197} \mathrm{Au}$ and ${ }^{208} \mathrm{~Pb}$ at $\mathrm{E}_{\mathrm{d}}=9 \mathrm{MeV}, Q_{\mathrm{h}}=40^{\circ}$.

\section{Fíg:-1은}

The "polarization integral" $I_{p}\left(Q_{r}\right)$ eq. (5.14) and its approximate value eq. (5.16) is shown as a function of the recoil momentum Qr.

\section{Fíg._-1느-}

The deviation between the coincidence cross section which includes the polarization effect and the one which neglects this effect is shown as a function of $E_{n}$ for different proton angles. The neutron angles are $\theta_{n}=20^{\circ}$ and $\theta_{n}=110^{\circ}$ $\operatorname{resp}_{;} \mathrm{E}_{\boldsymbol{d}}=9.5 \mathrm{MeV}$

\section{Fíg._-1 $\underline{5}$}

The same as in fig. 14 but for $E_{d}=11 \mathrm{MeV}$.

Fíg_-_므

The deviation between the pure Coulomb break-up cross section and the cross section which includes the polarization effect as a function of the recoil momentum (parametrized by $t_{r}$ ). The neutron energy is $0,5 \mathrm{MeV} ; E_{d}=9,5 \mathrm{MeV}$ and $11 \mathrm{MeV}$, resp.. The neutron angles are $\theta_{n}=20^{\circ}$ and $\theta_{n}=80^{\circ}$, resp.. The possible range of $t_{r}$ is indicated in the figure. 


\section{Fíg:-17}

The ratio $A_{2} /\left(q_{n}^{2} A_{0}\right)$ is shown as a function of $q_{n}$ for two different D-state probabilities $P_{D}(z)$ and three different hardcore radii.

\section{$\underline{\text { Fig }} \dot{-1} \underline{1} \underline{8}$}

Calculation of the deuteron break-up coincidence cross section on ${ }^{208} \mathrm{~Pb}$. The "pure coulomb" term is shown in a dashed line, whereas the full line was calculated according to eq. (5.34). The compound nucleus contribution is shown separately in a dotted line.

\section{Fig:- 19}

Comparison of our calculations with the data of ref. $[18]$. The same drawing conventions as in fig. 18 have been used.

\section{Fig $=-\underline{2} \underline{0}$}

The deviation $E\left(\theta, \xi, \xi^{\prime}, \rho, \eta\right)$ between semiclassical and the quantal calculations as a function of $1 / \eta^{2}$. The quantity $a_{c}$ has been kept fixed at $7 \mathrm{fm}$.

\section{Fig. $-\underline{2} \underline{1}$}

The break-up cross section, integrated over neutron angles, for $\theta_{p}=100^{\circ}$ as a function of the neutron energy at $E_{d}=9.5 \mathrm{MeV}$.

\section{Fig $=-\underline{2} 2$}

The same as in fig. 21 as a function of the neutron energy for different proton angles. 
Fig $\underline{\text { in }}-\underline{2} \underline{3}$

The same as in fig. 21 as a function of the proton angle, for diffe rent neutron energies.

Fìg:-_-2 $\underline{4}$

The energy spectrum of the emitted protons (neutrons) in the break-up reaction is presented for some deuteron energies $\mathrm{E}_{\mathrm{d}}$.

Eig._-_2

The total cross section of the break-up reaction on ${ }^{208} \mathrm{~Pb}$ as a function of the bombarding energy.

Fig._-26

The ratio Voo eq. (7.7) is shown as a function of the charge $\mathrm{Z}$ of the target nucleus for different neutron energies. The energy of the deuteron is $\mathrm{E}_{d}=10 \mathrm{MeV}$ and $12.5 \mathrm{MeV}$, respectively.

Fig._. 2 I

Comparison of the theoretical calculation for the "stripping enhancement factor" with the experimental results of ref. [119] for the reaction ${ }^{15} \mathrm{~N}(\mathrm{~d}, \mathrm{p}){ }^{16} \mathrm{~N}$ (unbound).

$\underline{\text { Fig._-2 }} \underline{8}$

The same as in fig. 27 for the reaction ${ }^{24} \mathrm{Mg}(\mathrm{d}, \mathrm{p}){ }^{25} \mathrm{Mg}$ (unbound). The experimental results are taken from ref。 $[120]$. 


\section{Fíg__-29}

Comparison of the calculations using "model SC"(right-hand side) for the reaction ${ }^{15} \mathrm{~N}(\mathrm{~d}, \mathrm{p}){ }^{16} \mathrm{~N}$ (unbound) with the experimental results of refs. $[34,35]$ (left-hand side).

\section{Fig. $3 \underline{30}$}

Model calculations for the ${ }^{15} \mathrm{~N}(\mathrm{~d}, \mathrm{p}){ }^{16} \mathrm{~N}$ continuum stripping spectrum. Three different curves are shown, corresponding to "model VC"(dashed line)," model SC"(continuous line) and a calculation with a "cut-off" (dashed-dotted line).

\section{Fig._- $\underline{3} \underline{1}$}

Schematic interpretation of the energy dependence of $d^{2} \sigma / d \Omega_{p} d E_{n}$.

\section{Fig._- $\underline{32}$}

A model calculation for ${ }^{208} \mathrm{~Pb}(\mathrm{~d}, \mathrm{p}){ }^{209} \mathrm{~Pb}$ (unbound) for several different Q- values. We have chosen $l_{\eta}=2$ and $E_{d}=11 \mathrm{MeV}$.

\section{Fíg._3 3}

The dependence of unbound state stripping on the angular momentum $l_{n}$. We have assumed that $\Gamma_{l_{n}}=$ const $=1 \mathrm{keV}$. We have chosen the reaction ${ }^{208} \mathrm{~Pb}(\mathrm{~d}, \mathrm{p}){ }^{209}$ (unbound) at $\mathrm{E}_{d}=11 \mathrm{MeV}$ and $Q=-2,5 \mathrm{MeV}$.

\section{Fig. $3 \underline{3}$}

The same as in fig. 33 but for $Q=3.5 \mathrm{MeV}$. 
- VII -

Fig._- $\underline{3} \underline{5}$

Comparison of different model calculations for the reaction ${ }^{208} \mathrm{~Pb}(\mathrm{~d}, \mathrm{p}){ }^{209} \mathrm{~Pb}$ (unbound) for $\mathrm{E}_{\mathrm{d}}=12 \mathrm{MeV}$ with the experimental data of ref. 130 . 


\section{Acknowledgements}

It is a great pleasure for us to thank Drs. F. Rösel and M. Pauli who worked with us on the present subject in various phases. Their fruitful collaboration formed a great amount of this report. Furthermore, we would like to thank Profs. K. Alder, A. Faessler, H.A. Weidenmüller and A. Winther for all the many discussions, their criticism and support. We would also like to thank Dr. G.R. Plattner for his careful reading of the manuscript. 


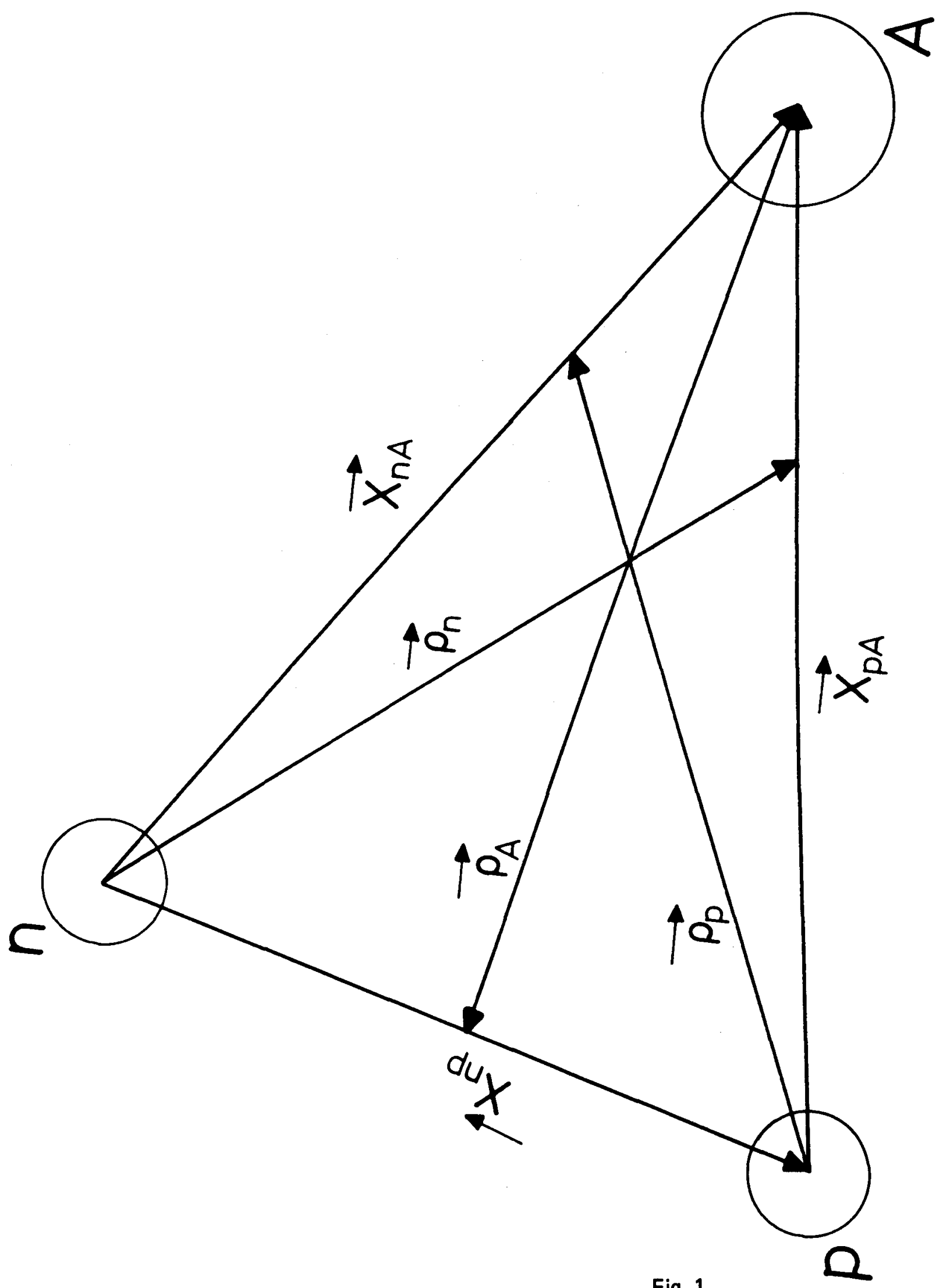

Fig. 1 


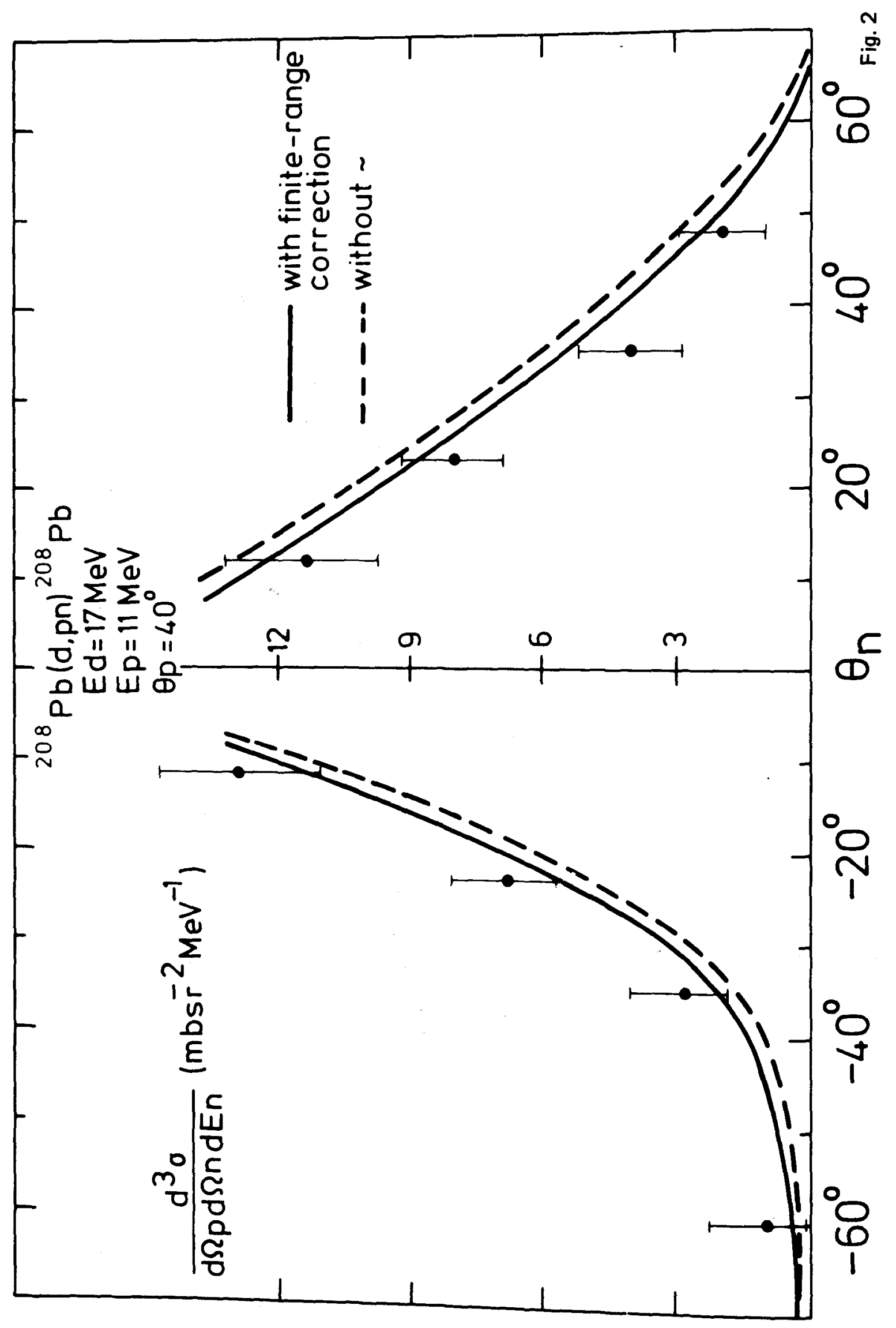




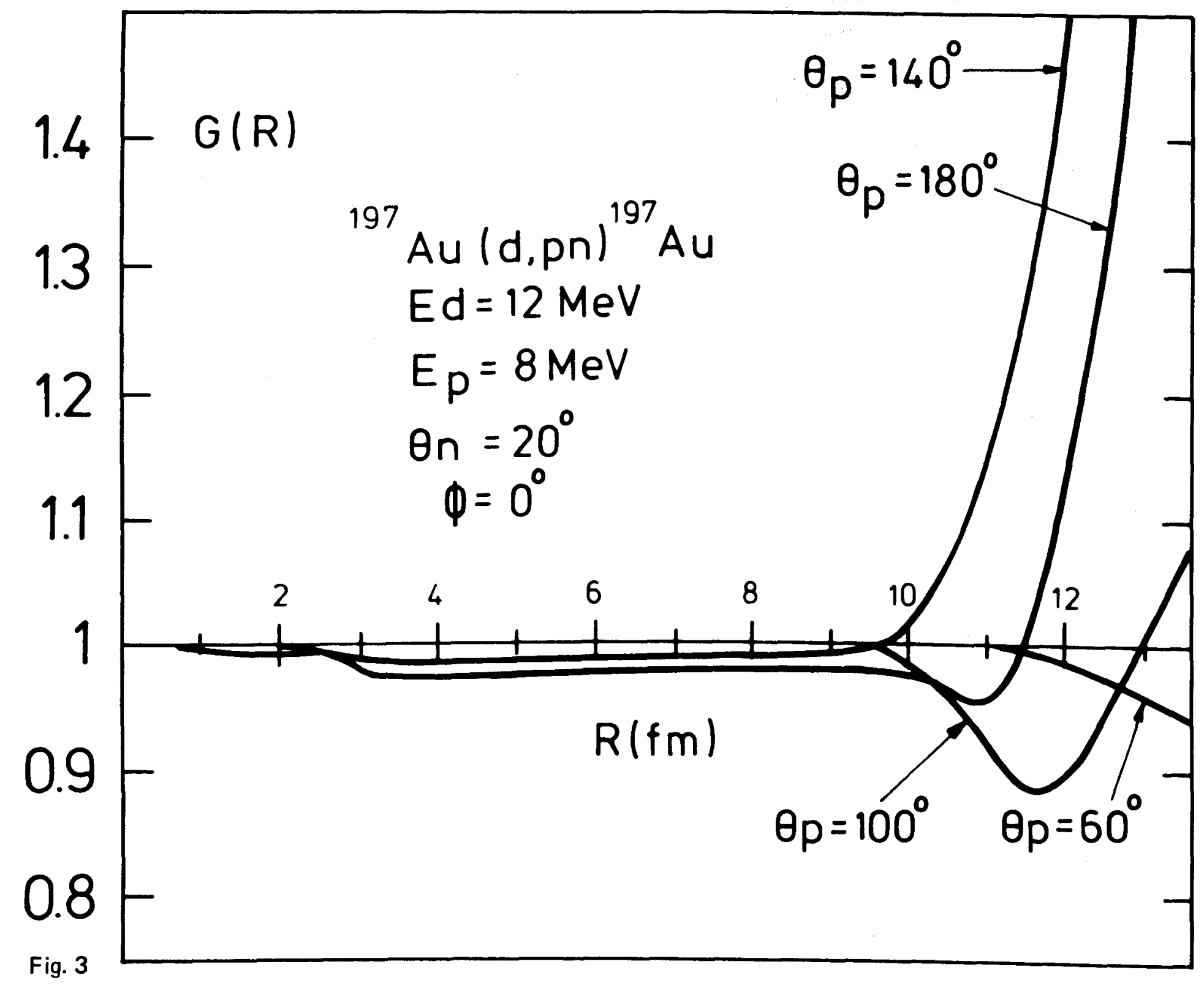




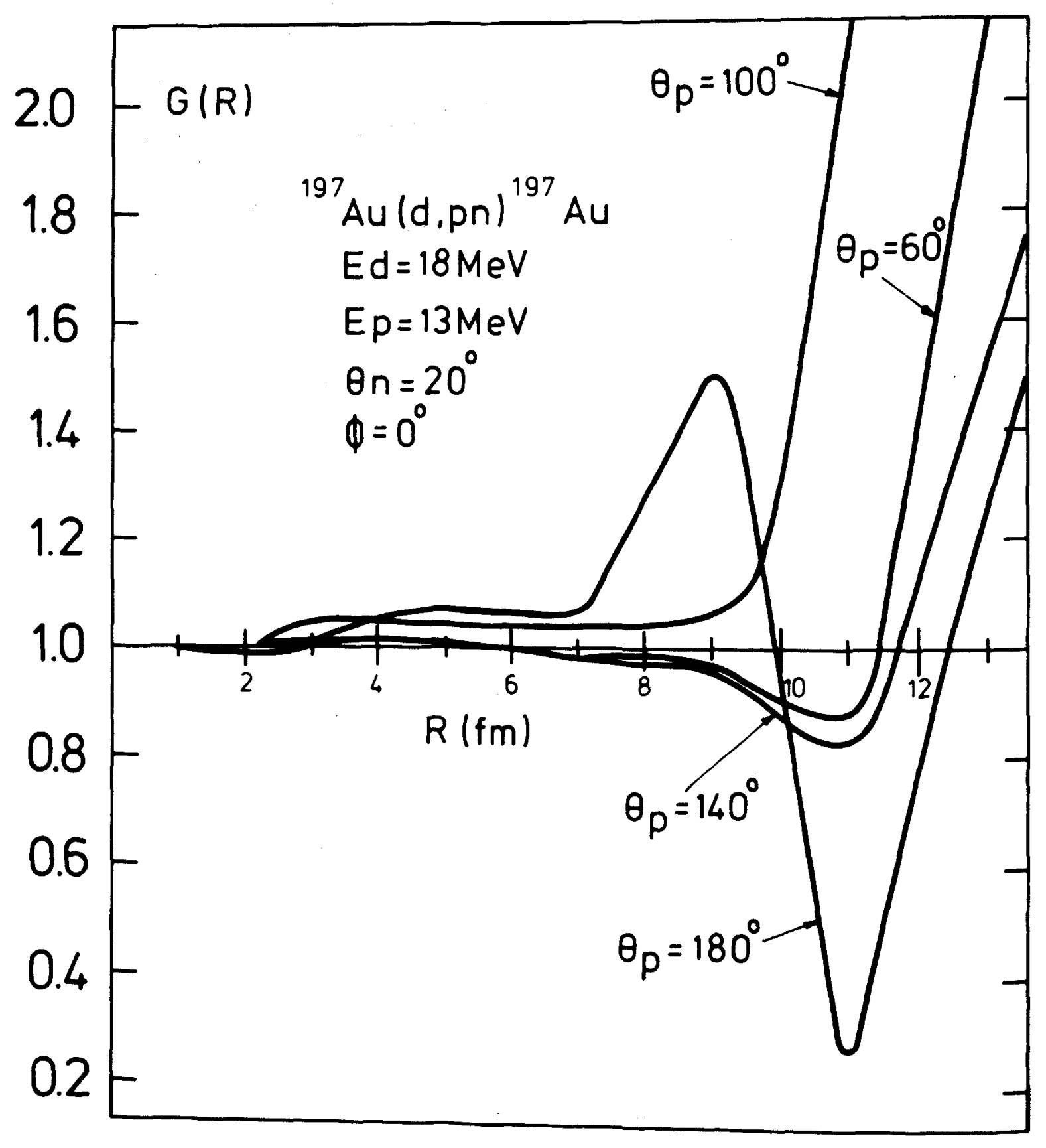

Fig. 4 


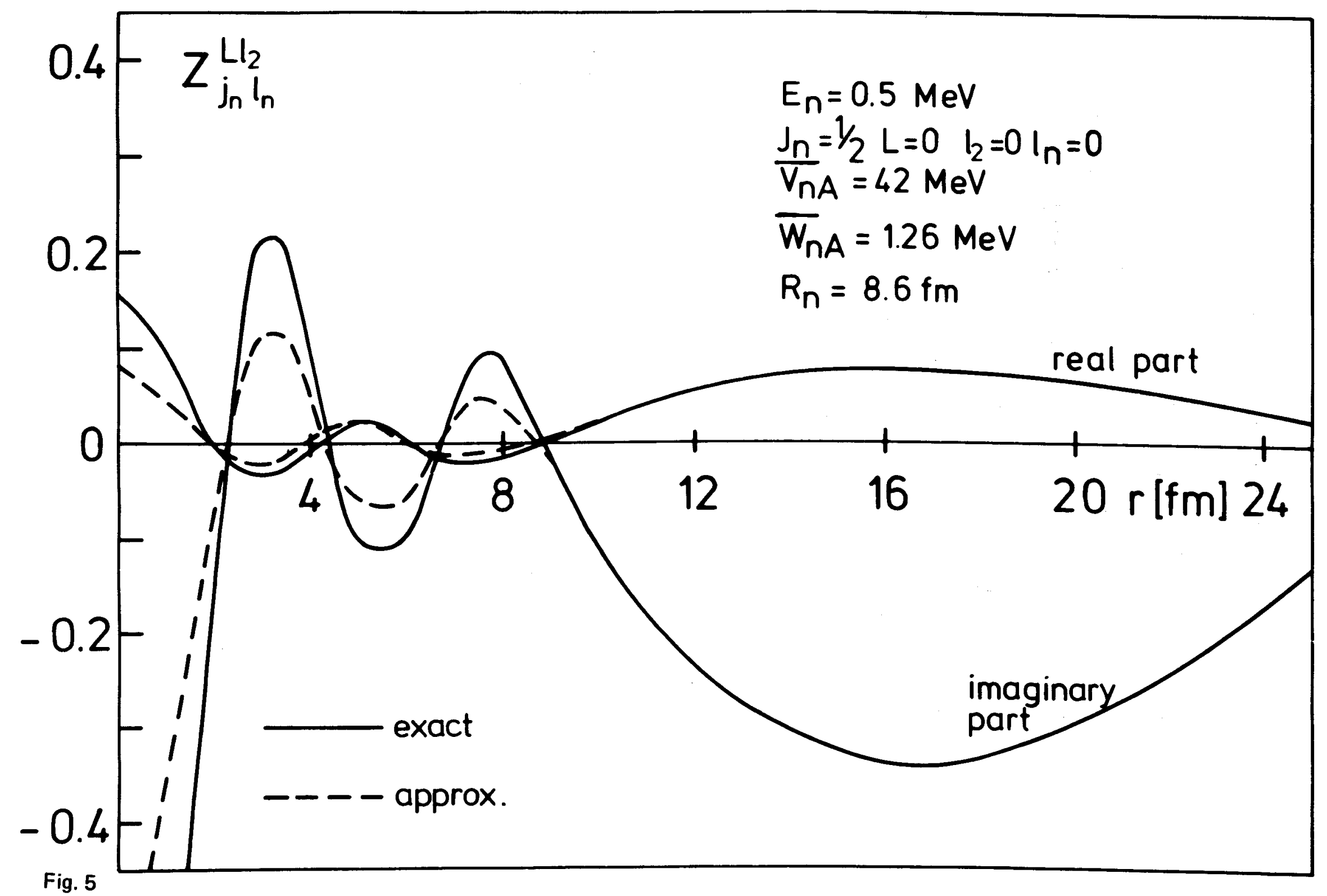




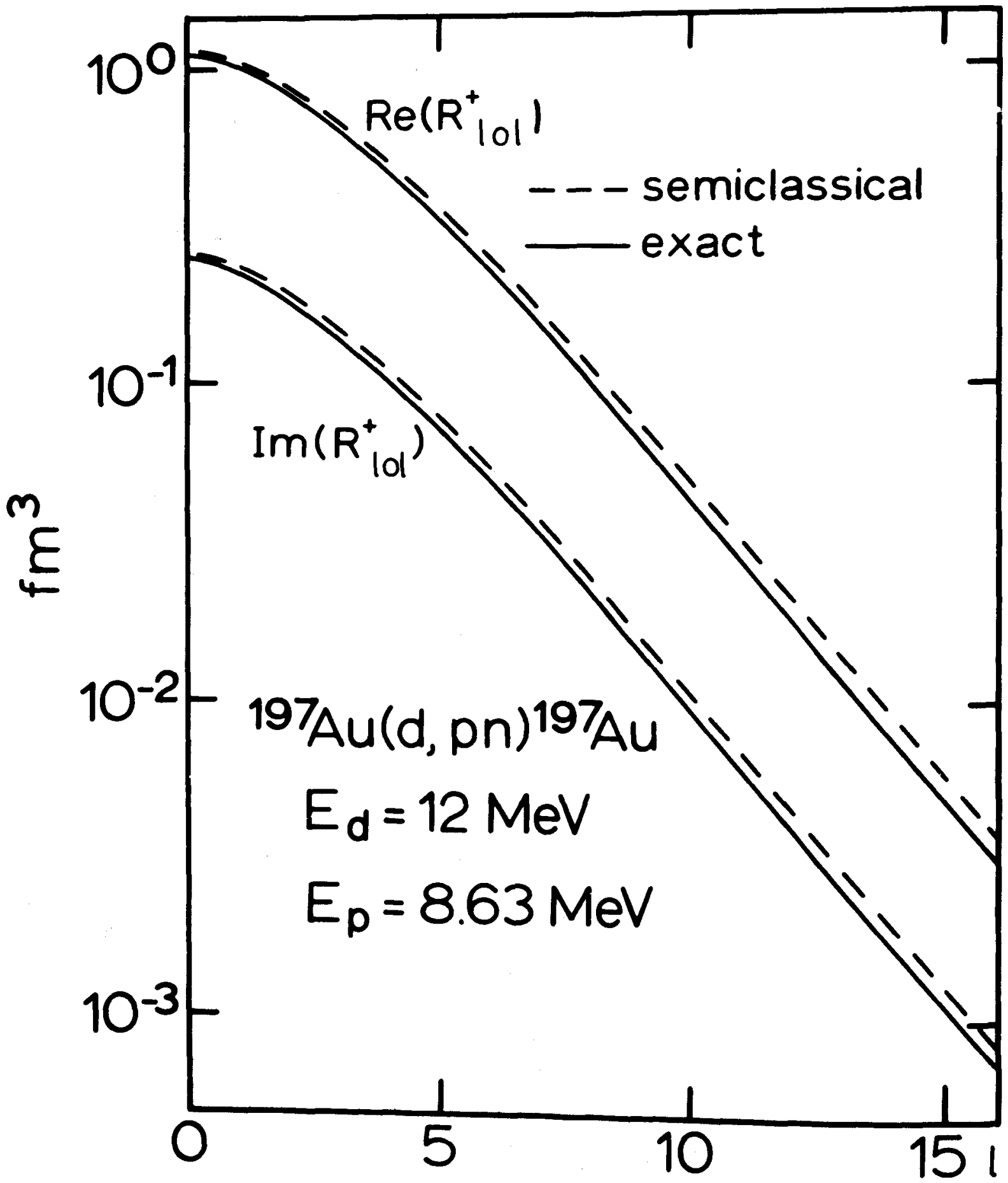

Fig. 6 


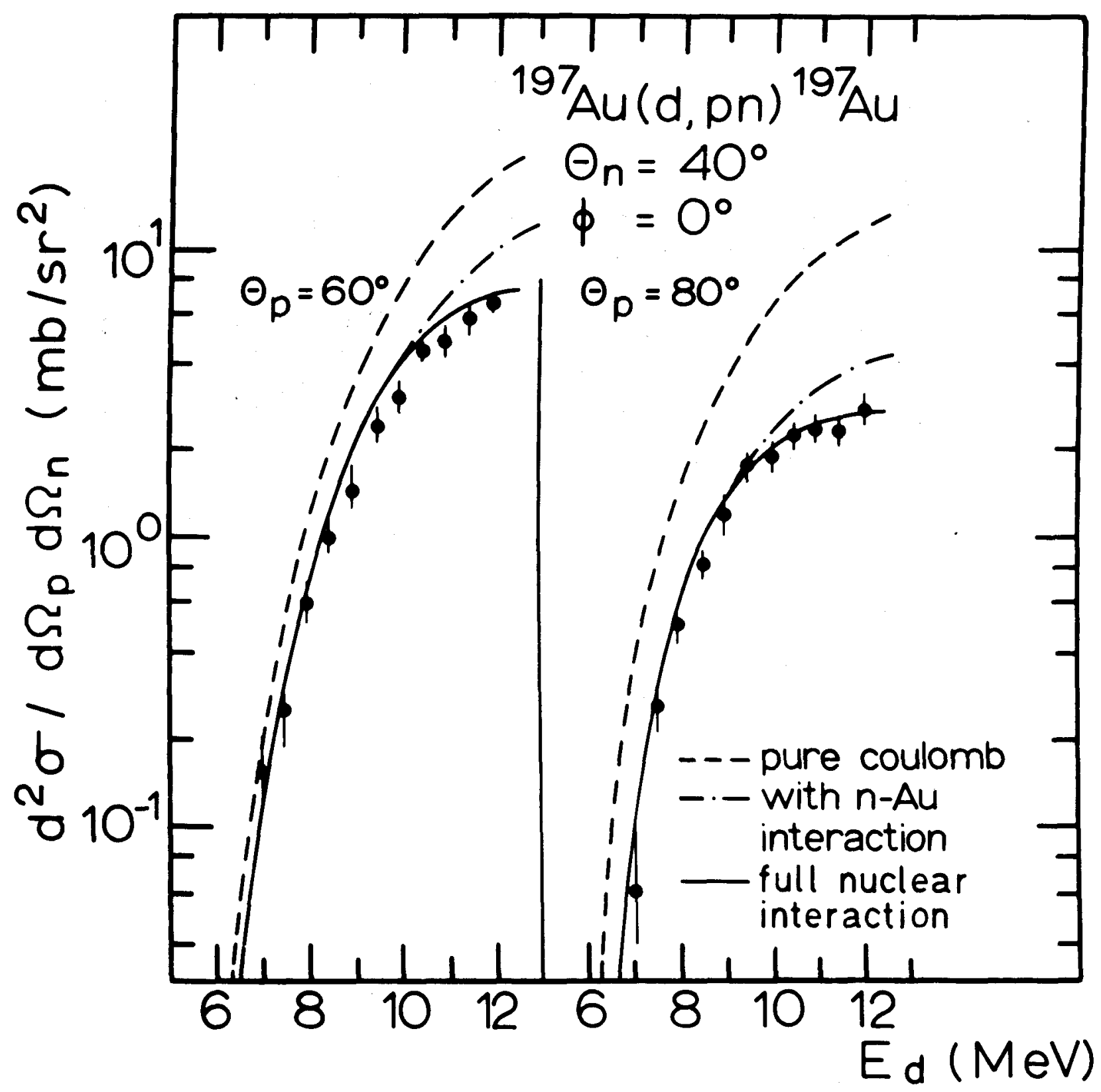

Fig. 7 


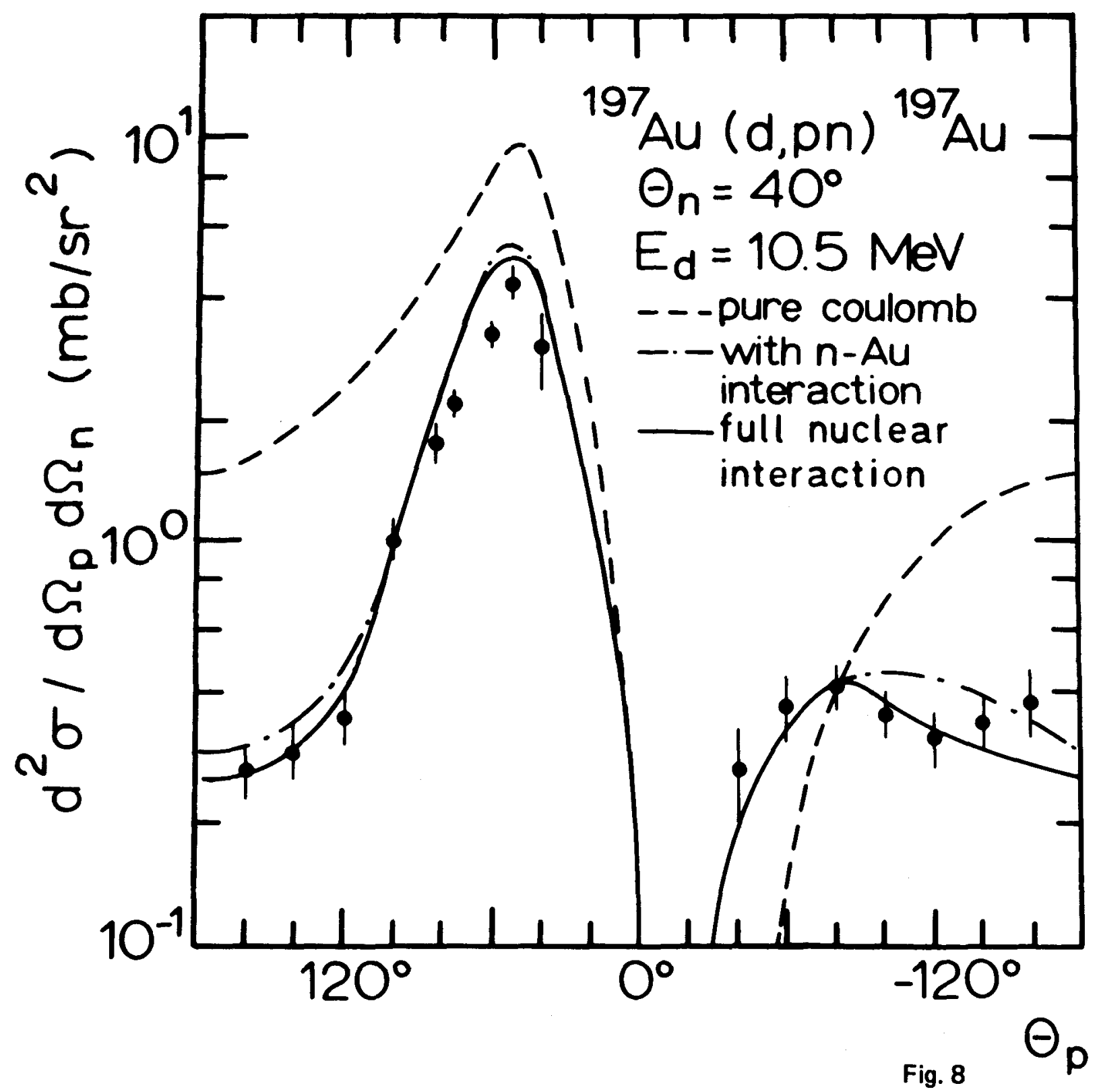




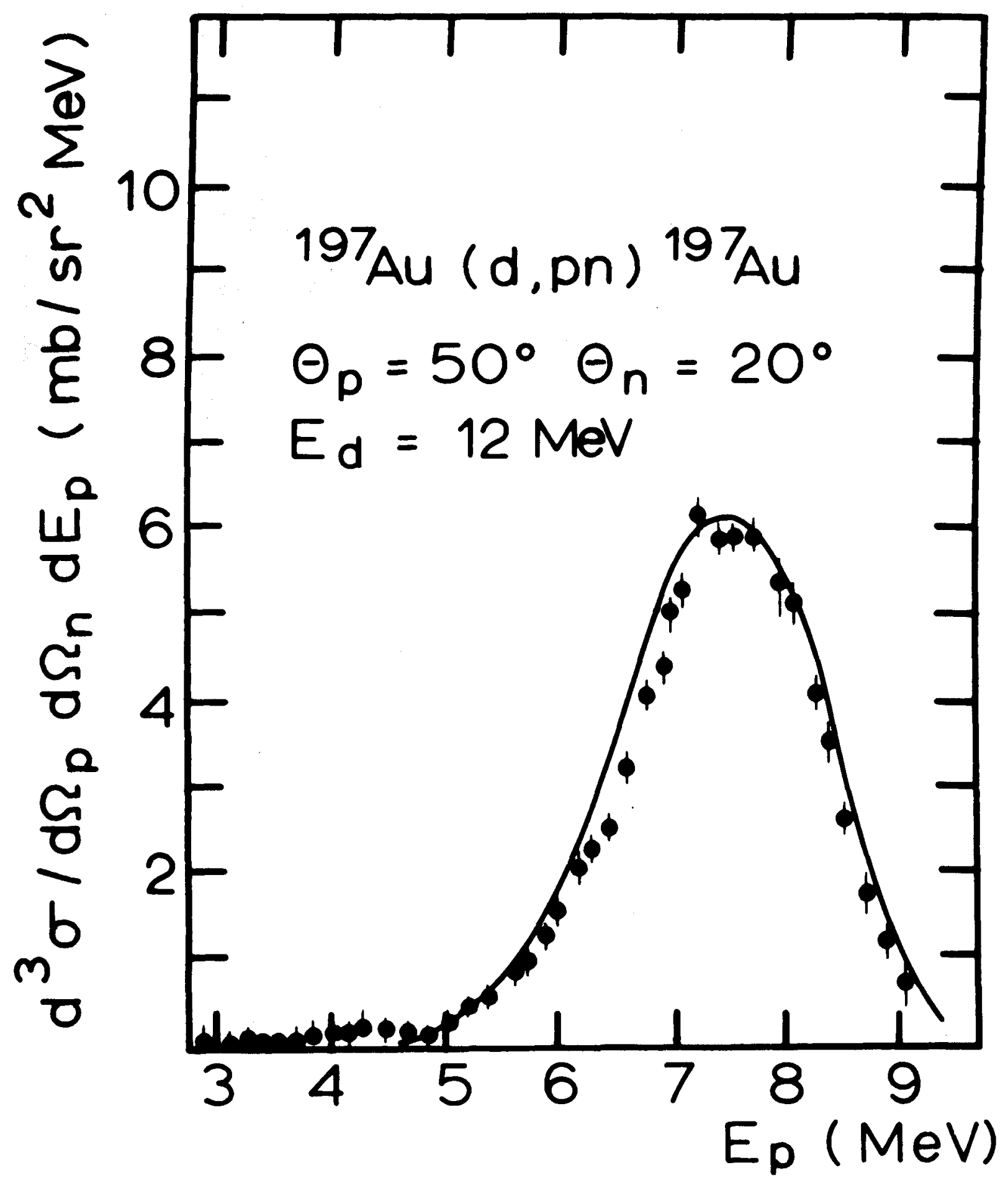

Fig. 9 


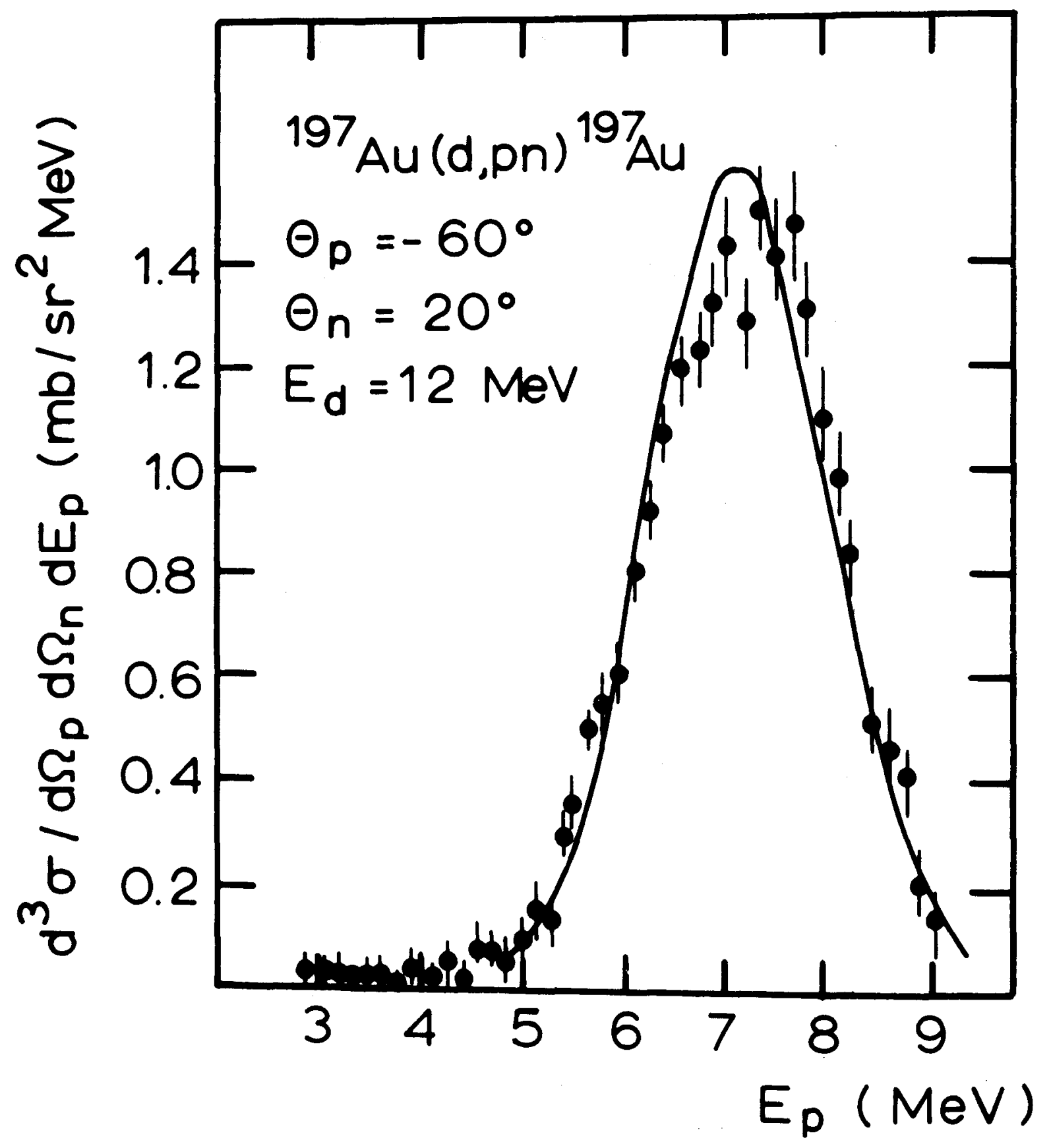

Fig. 10 


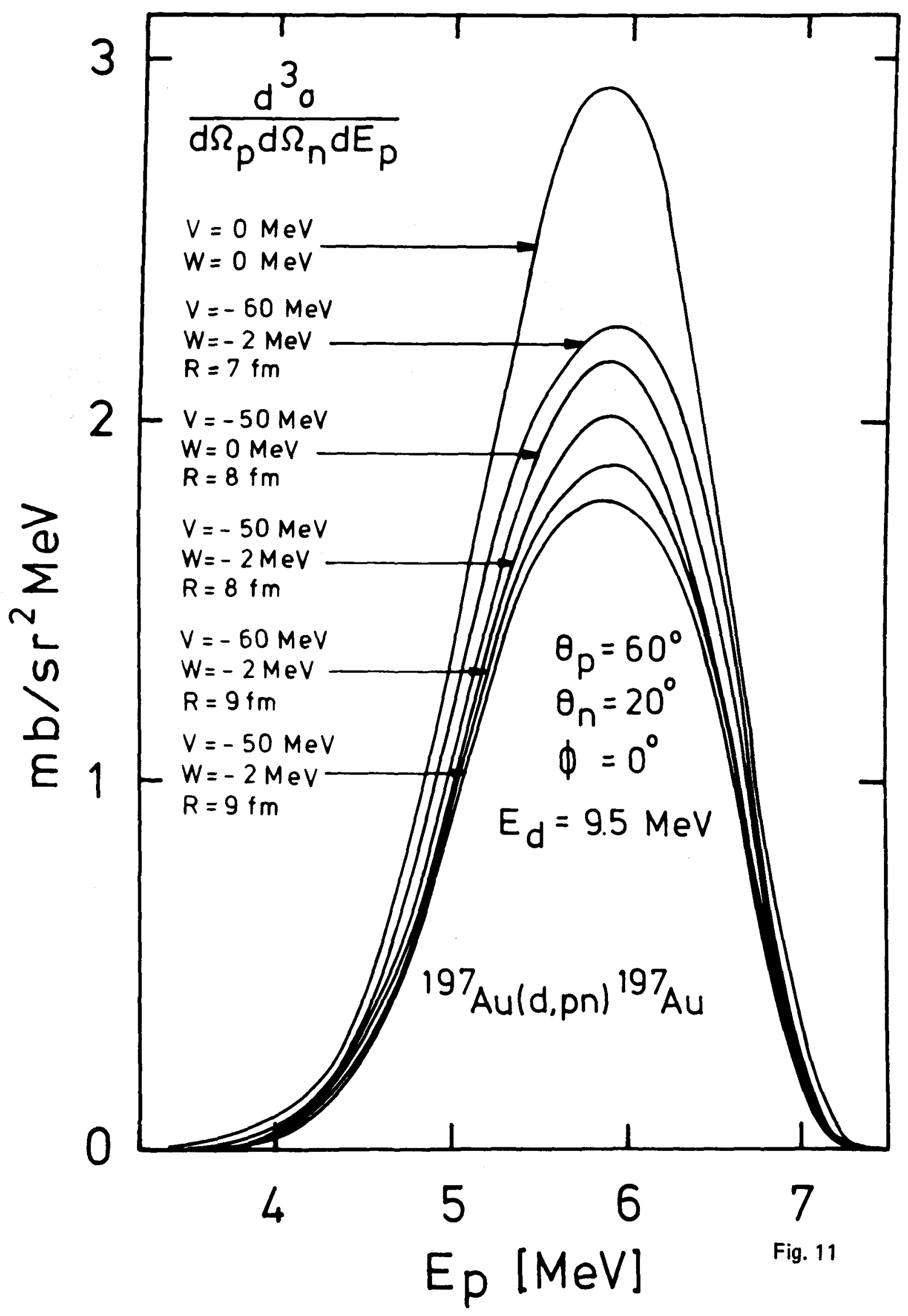




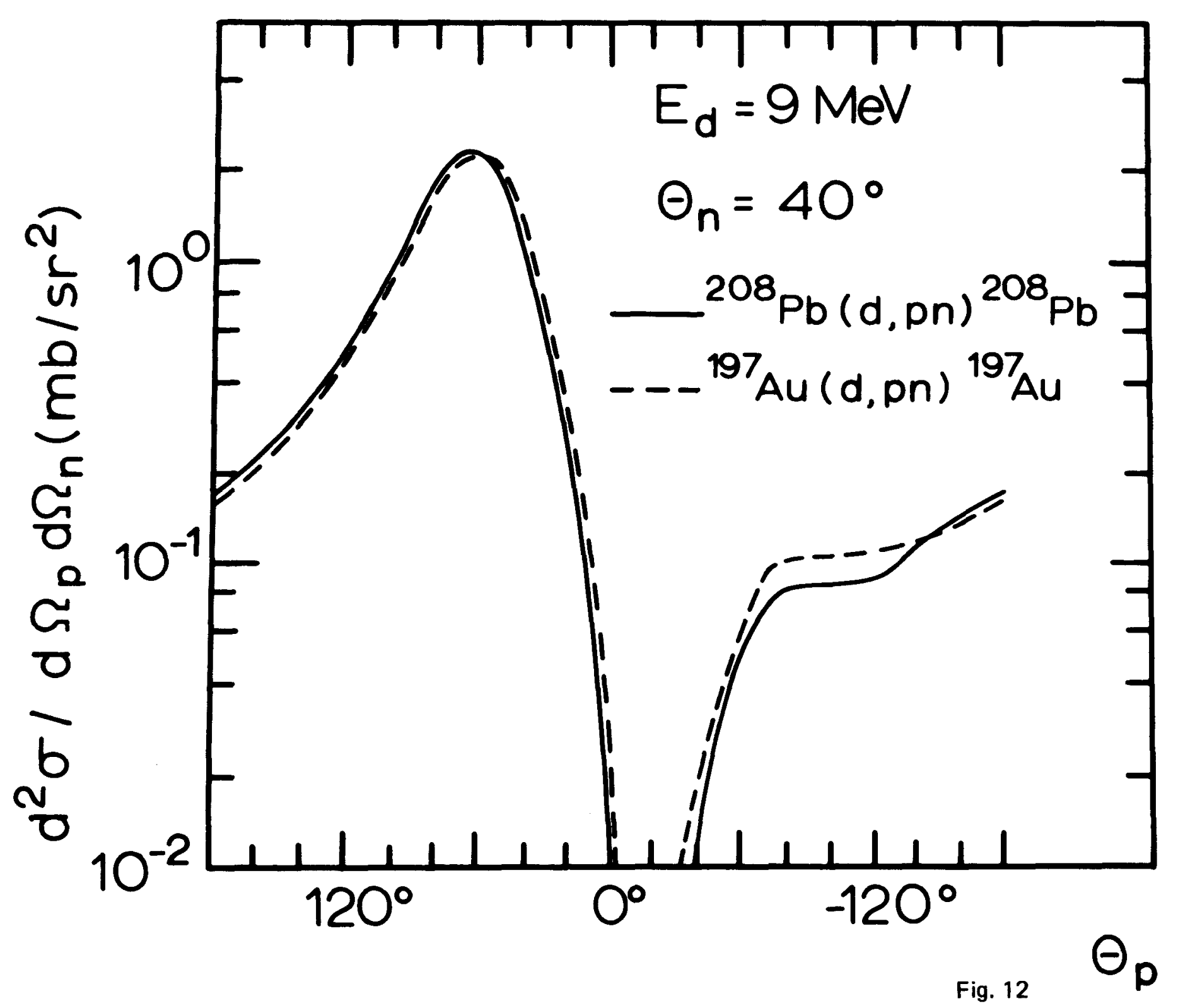




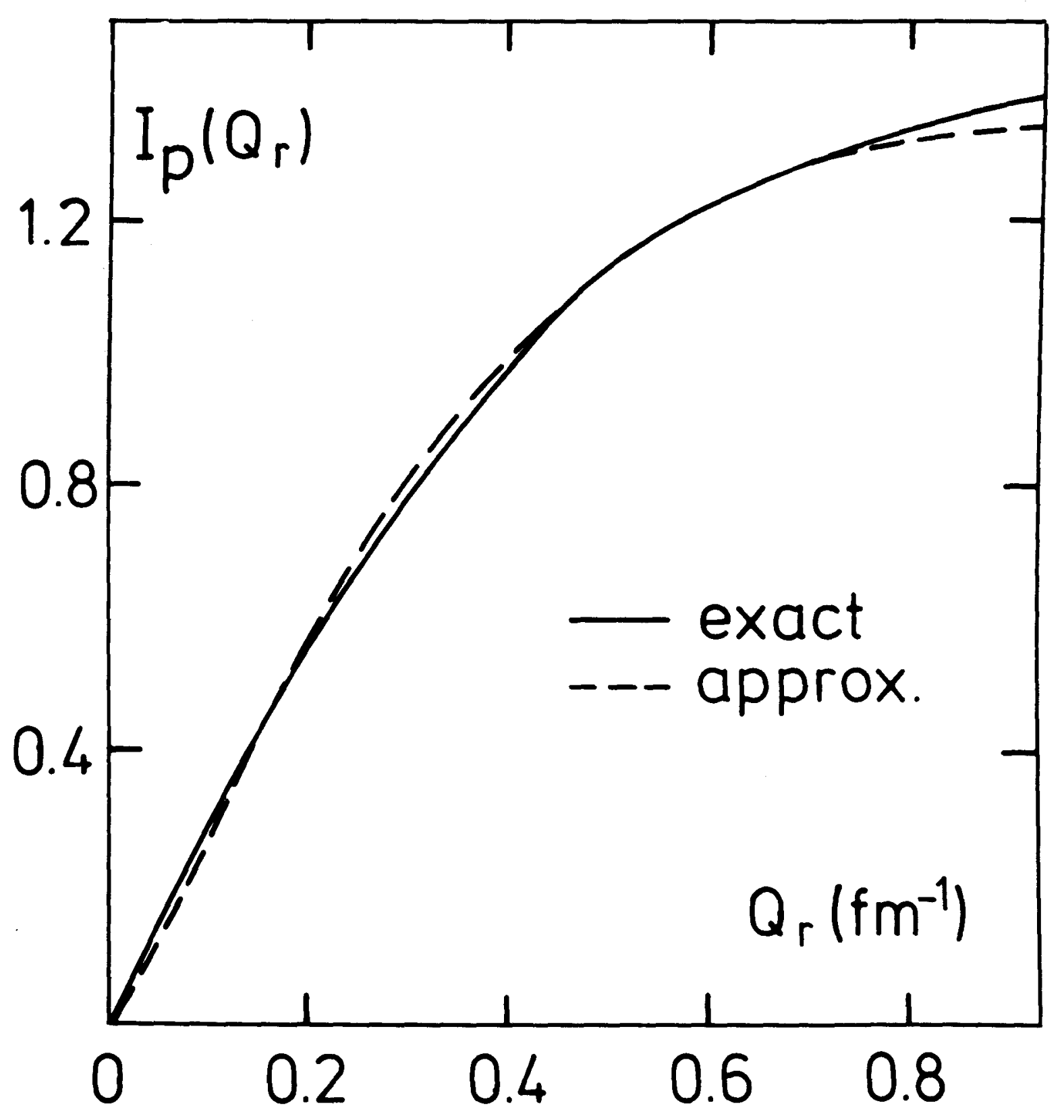

Fig. 13 


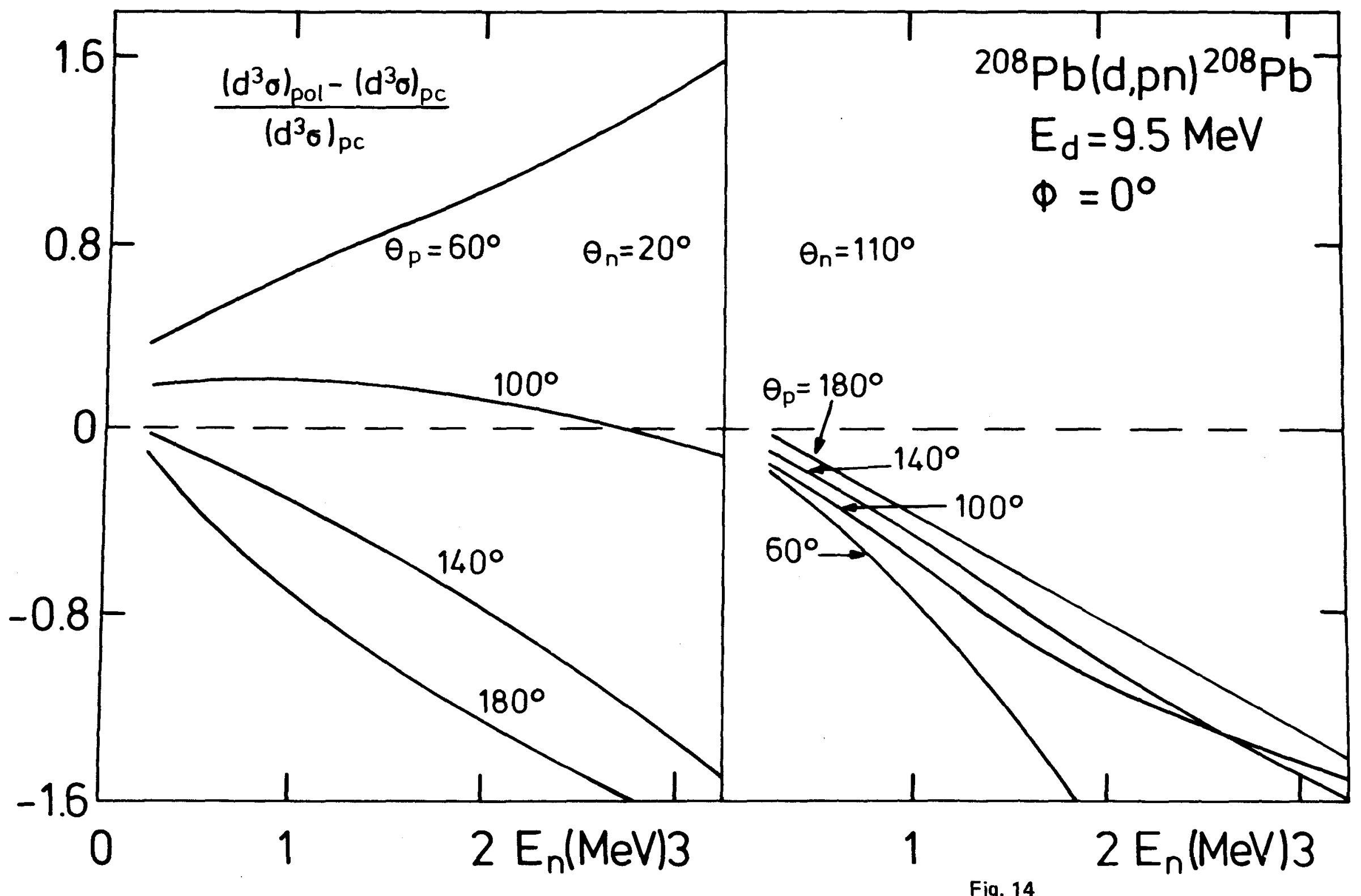

Fig. 14 


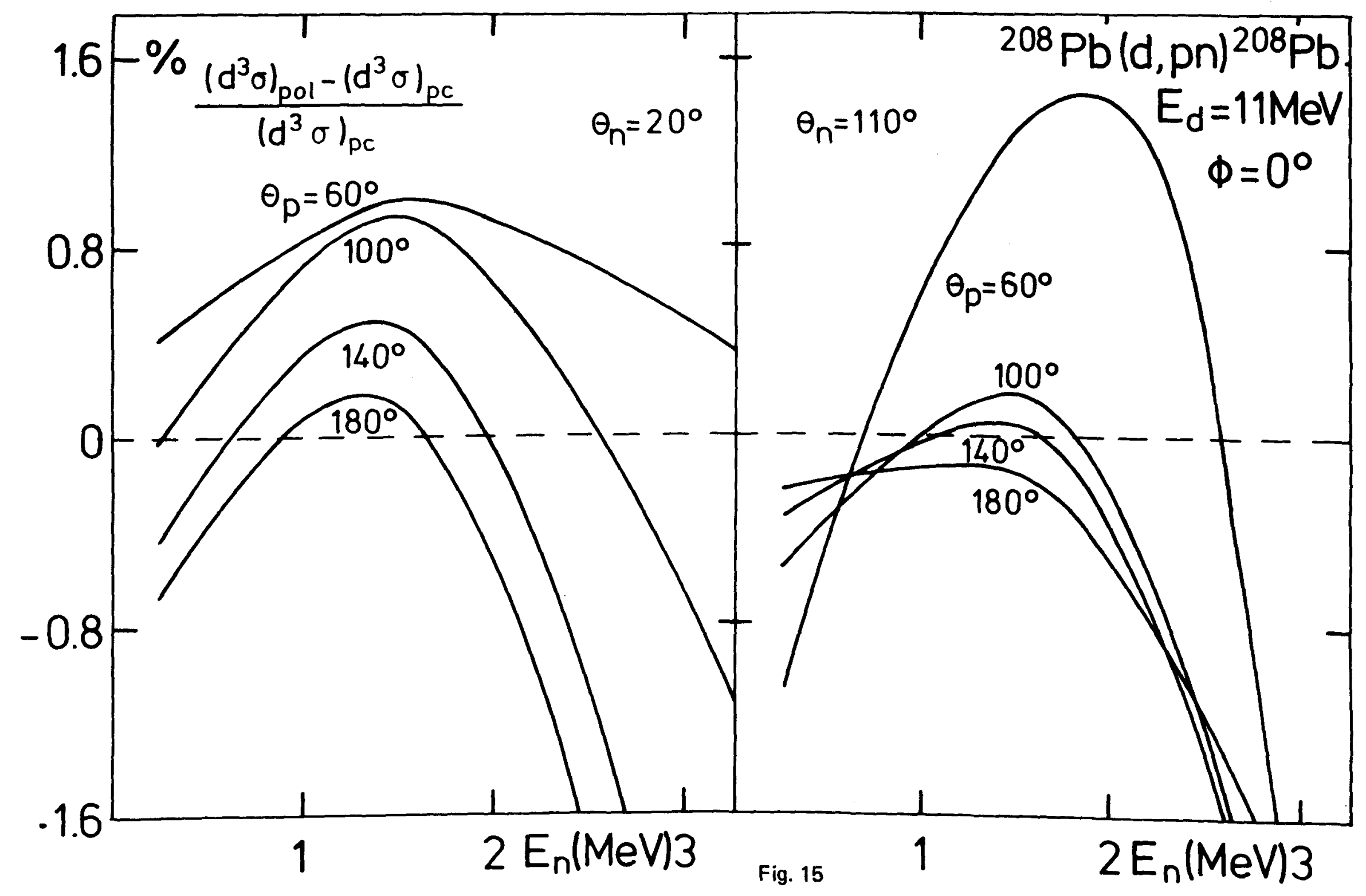




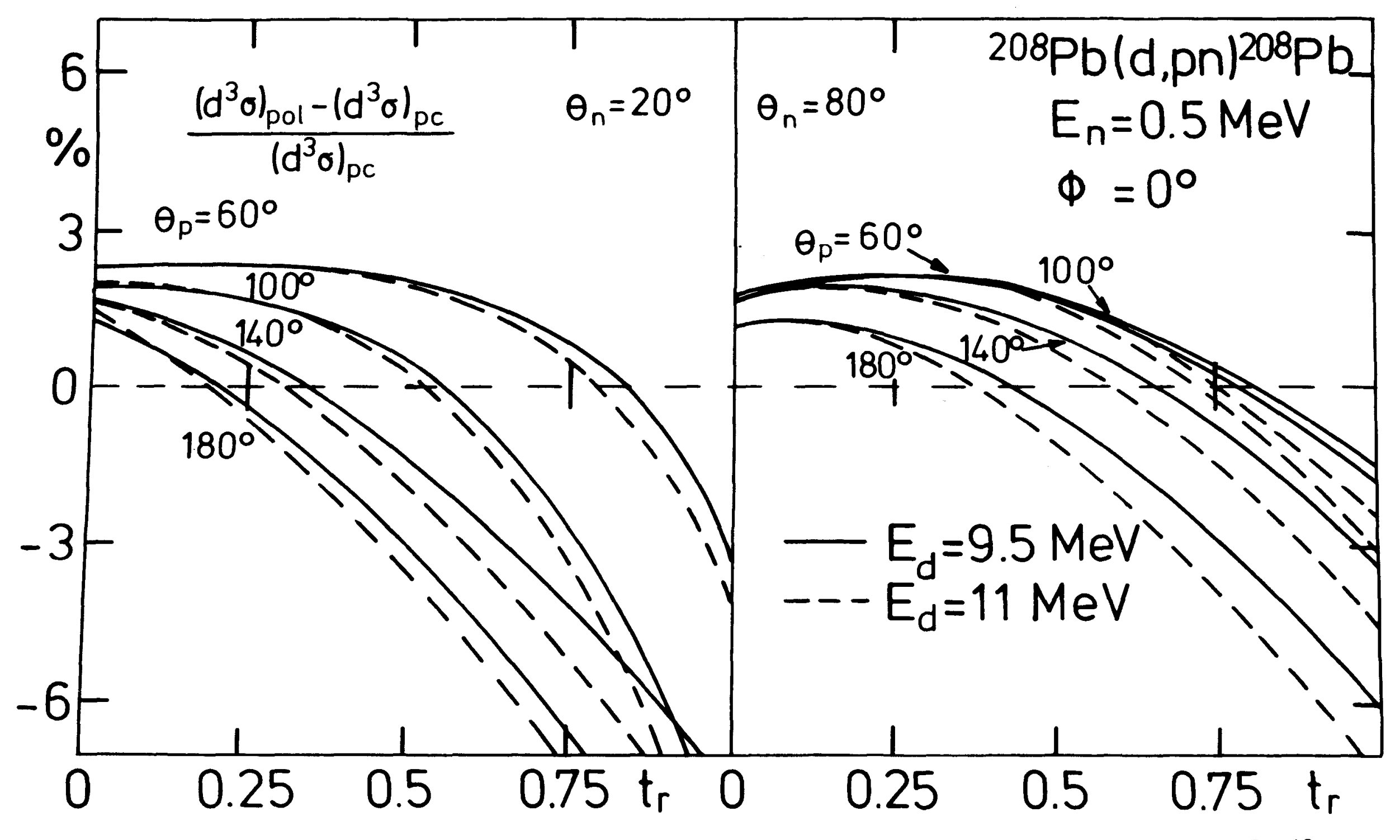

Fig. 16 


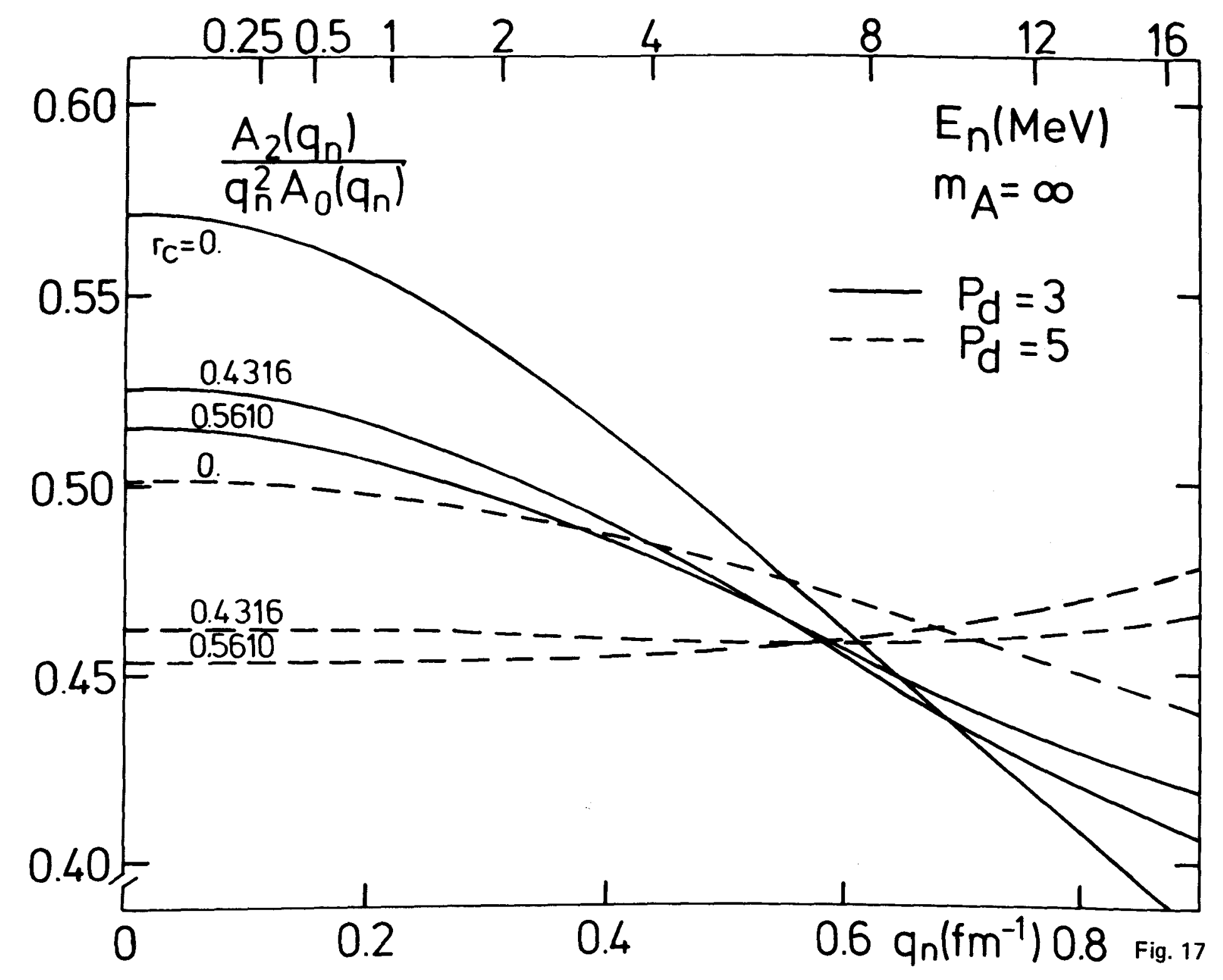




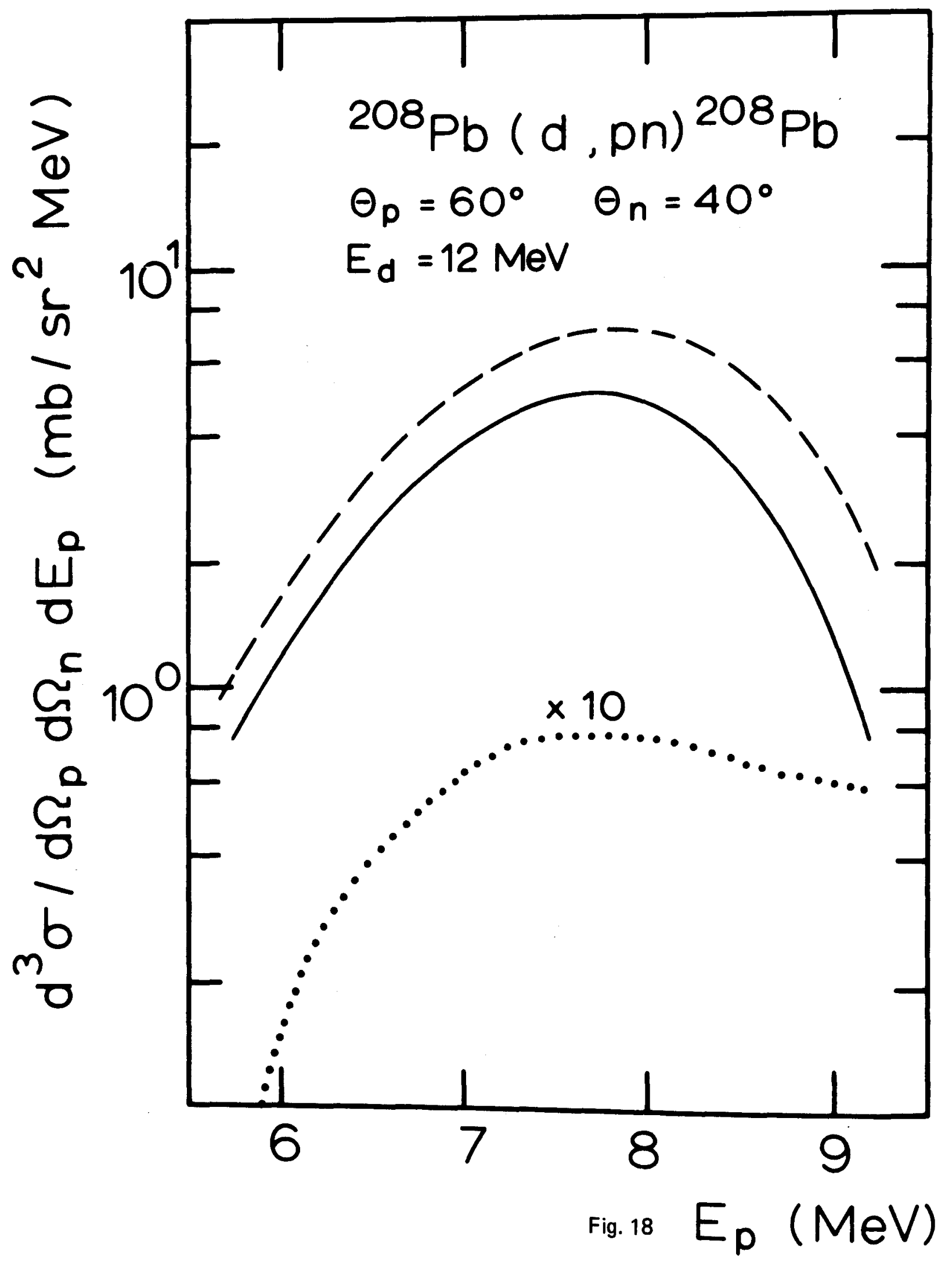




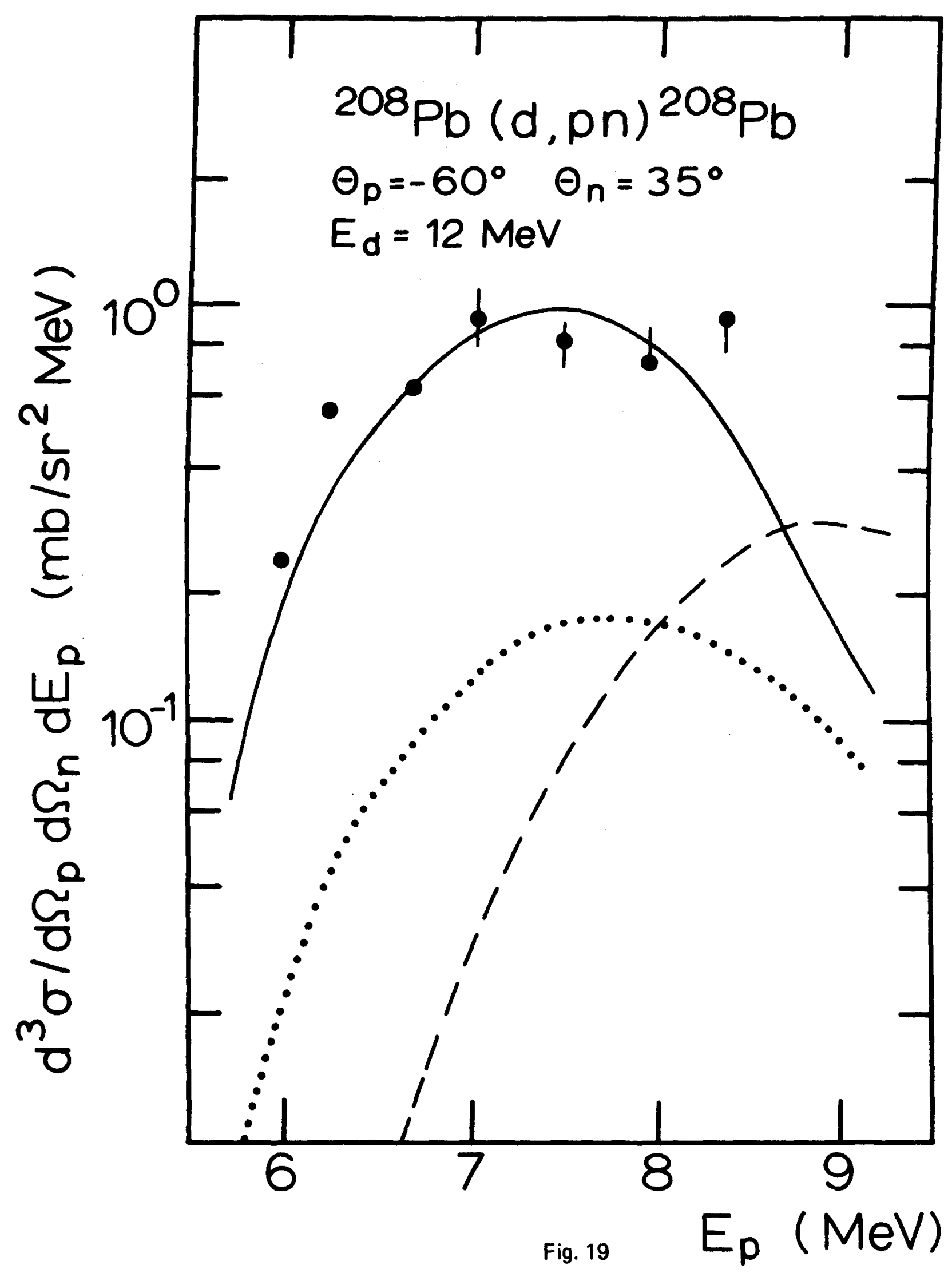




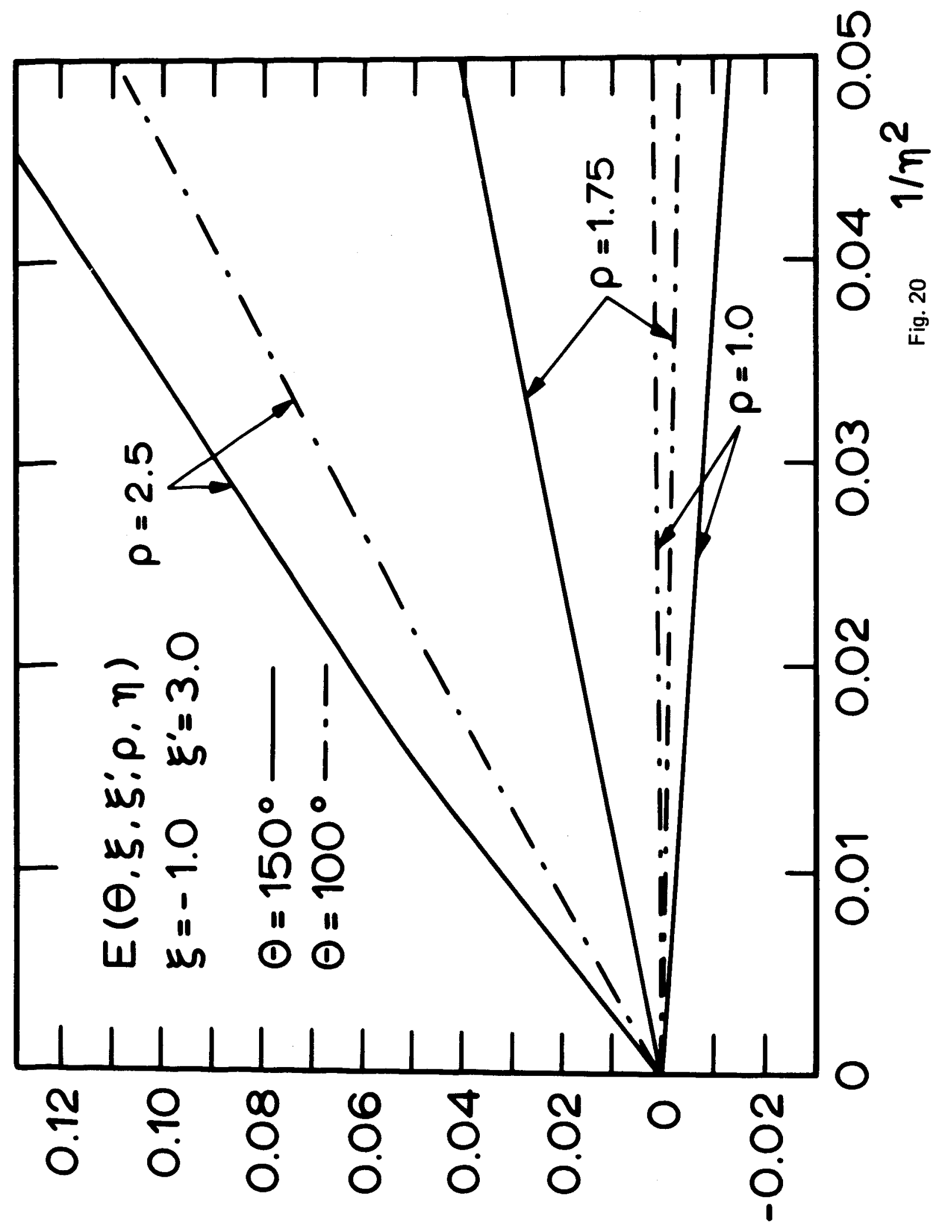




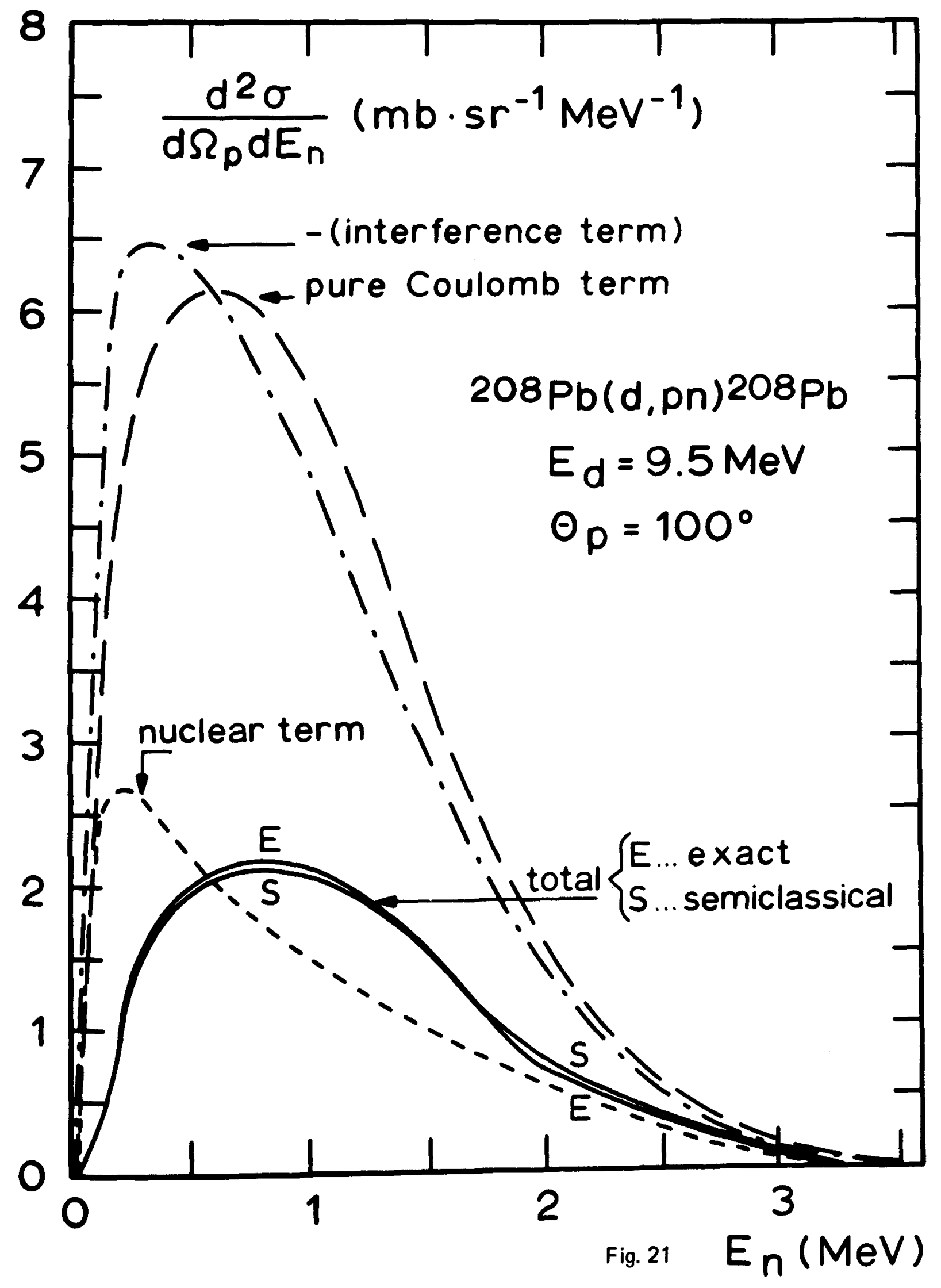




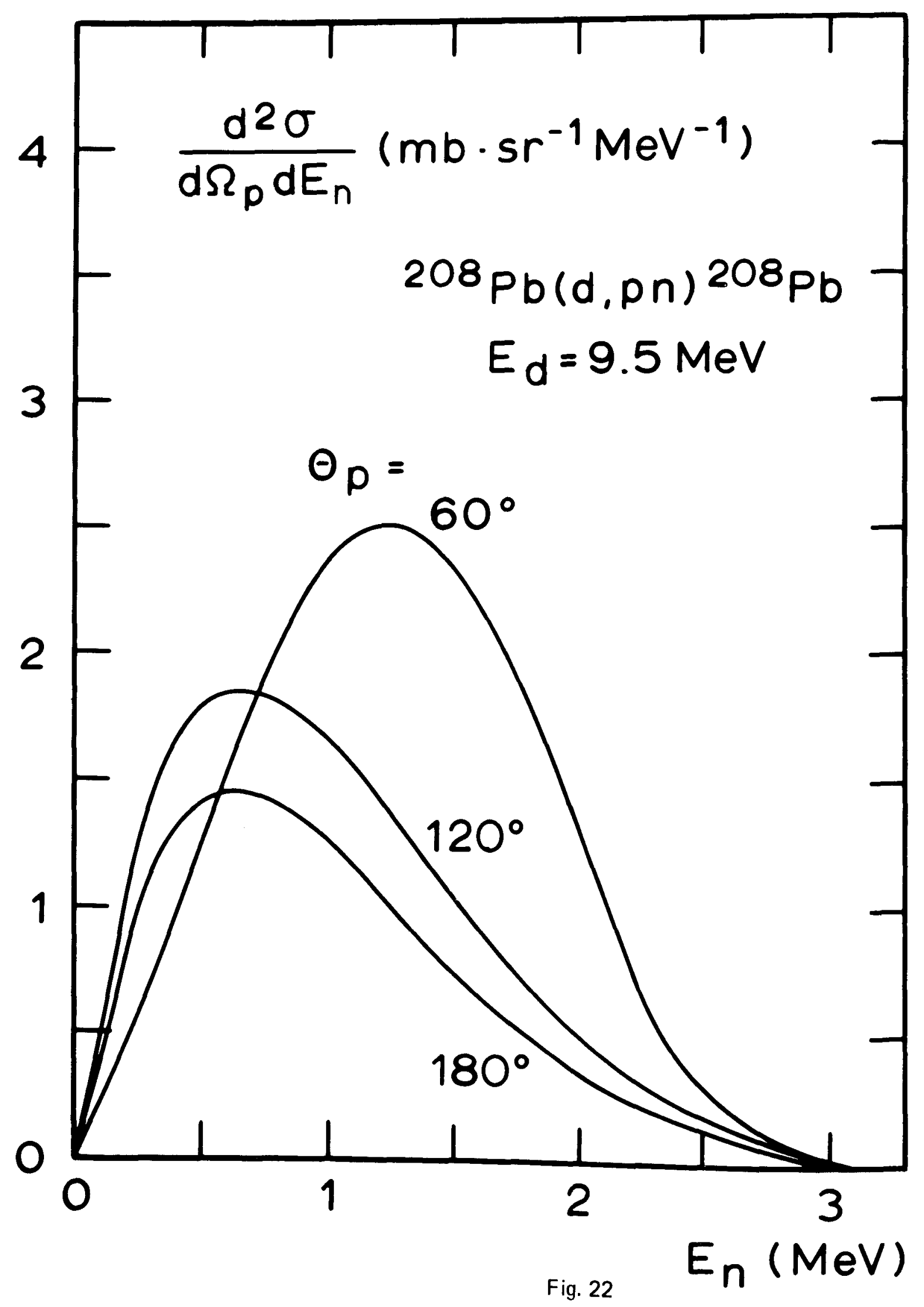




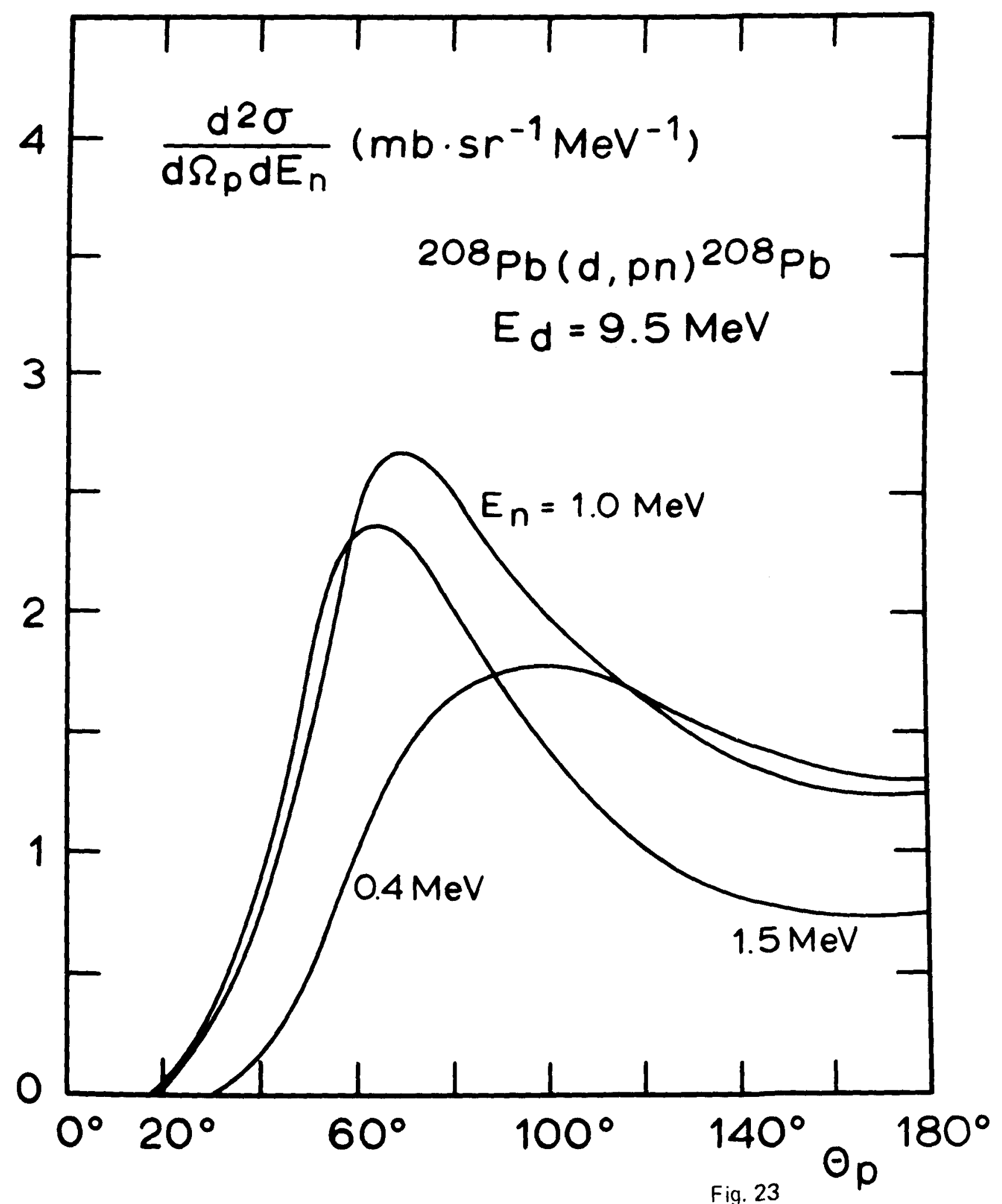

Fig. 23 


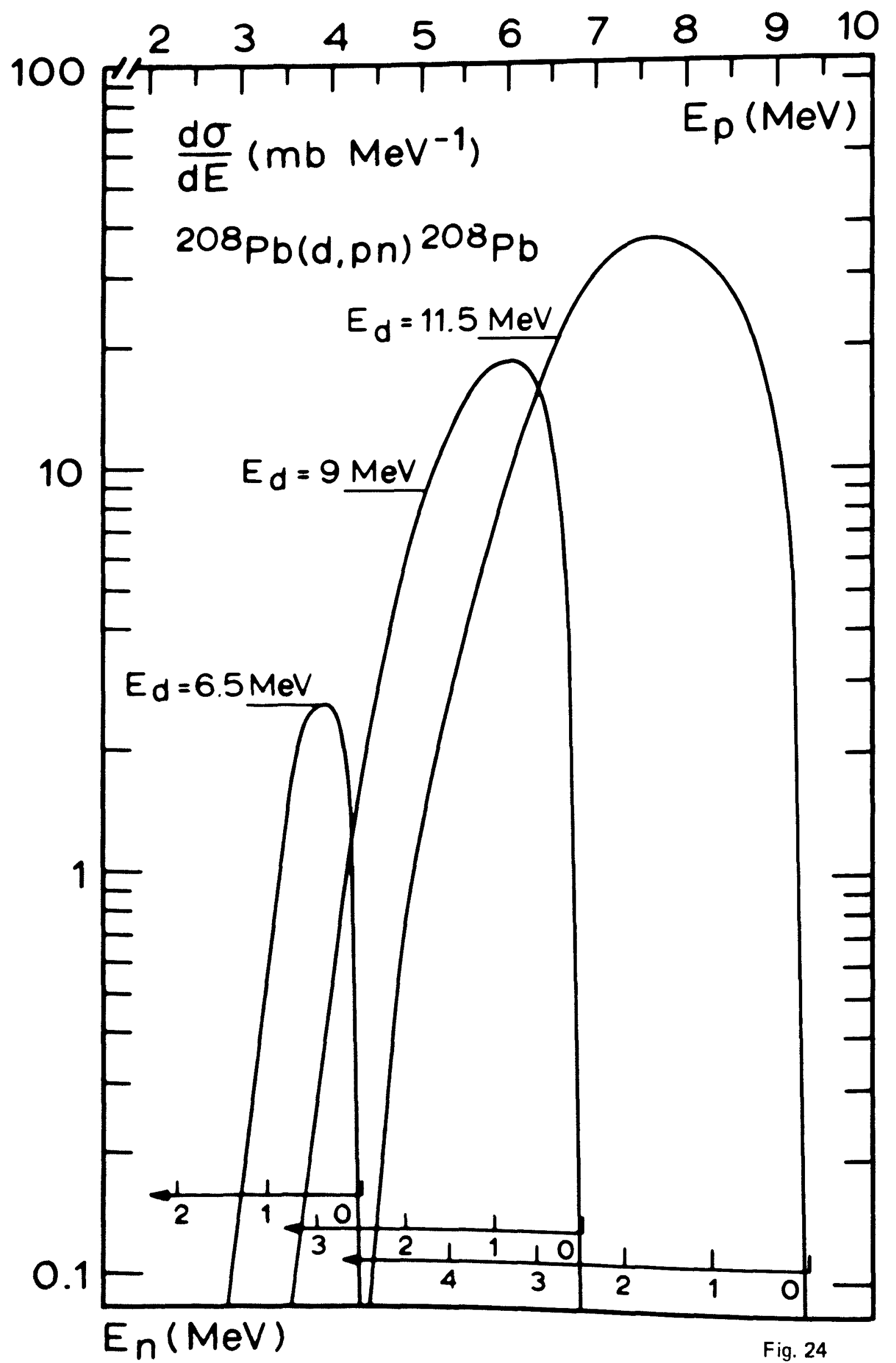




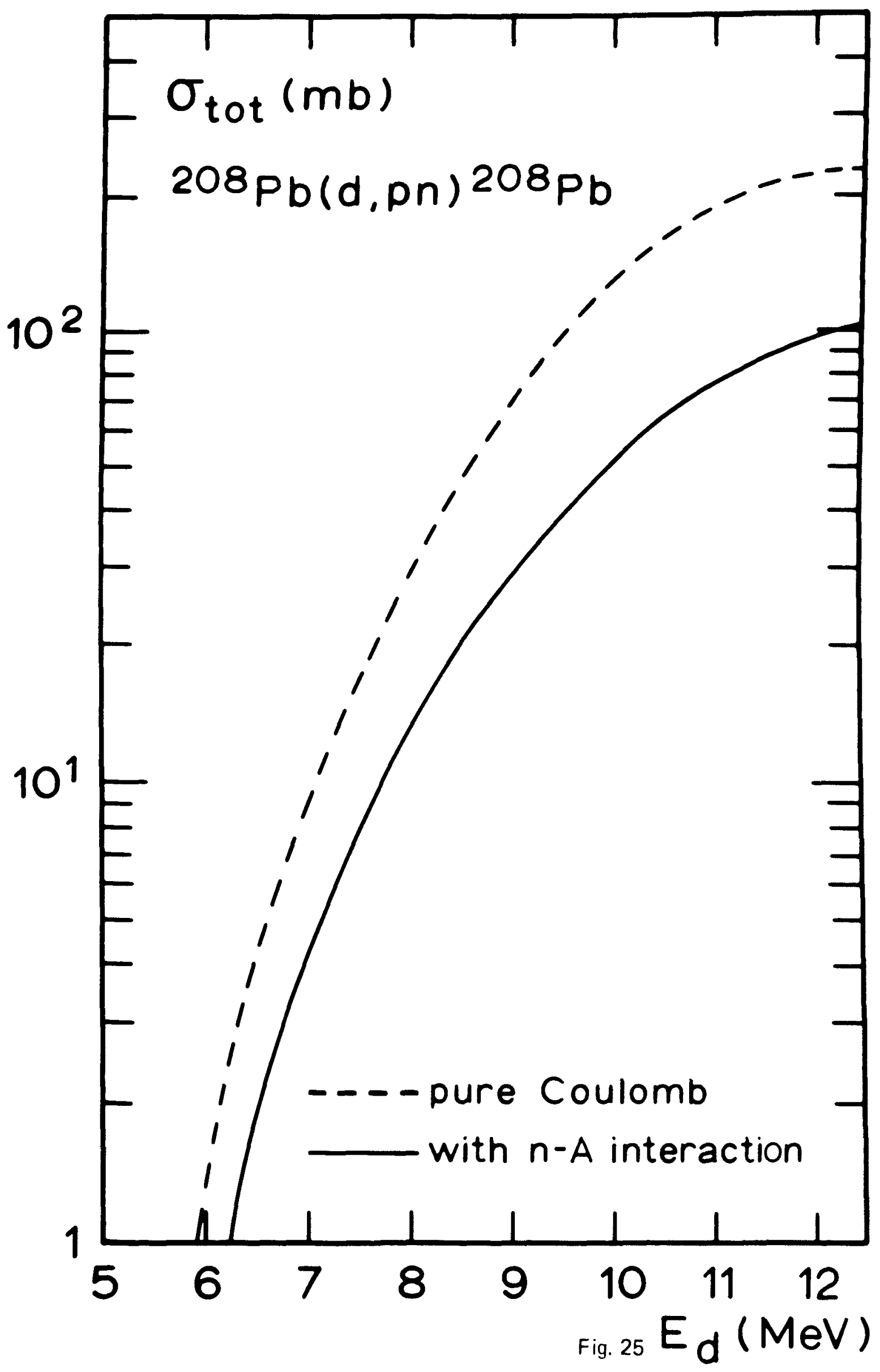




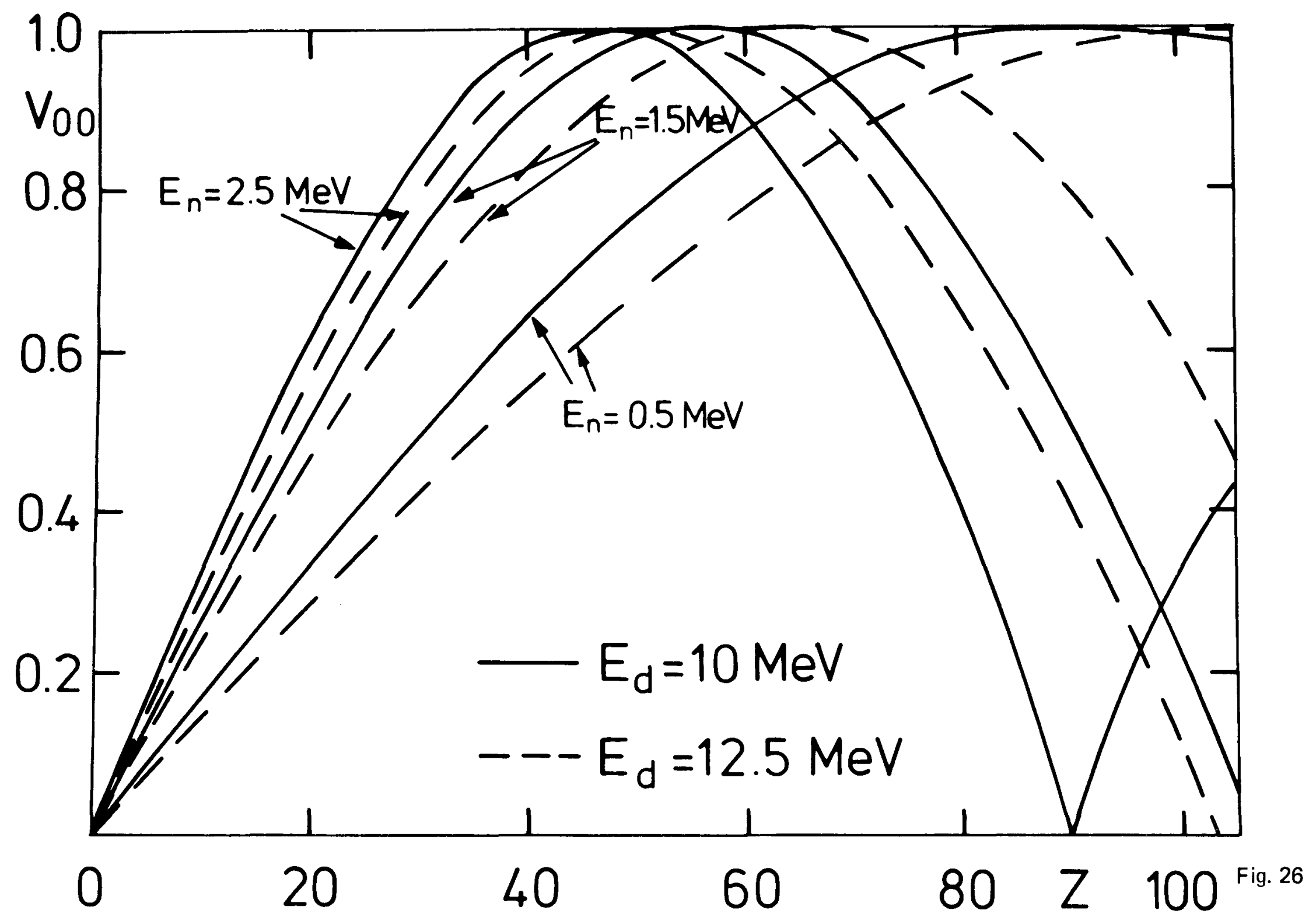




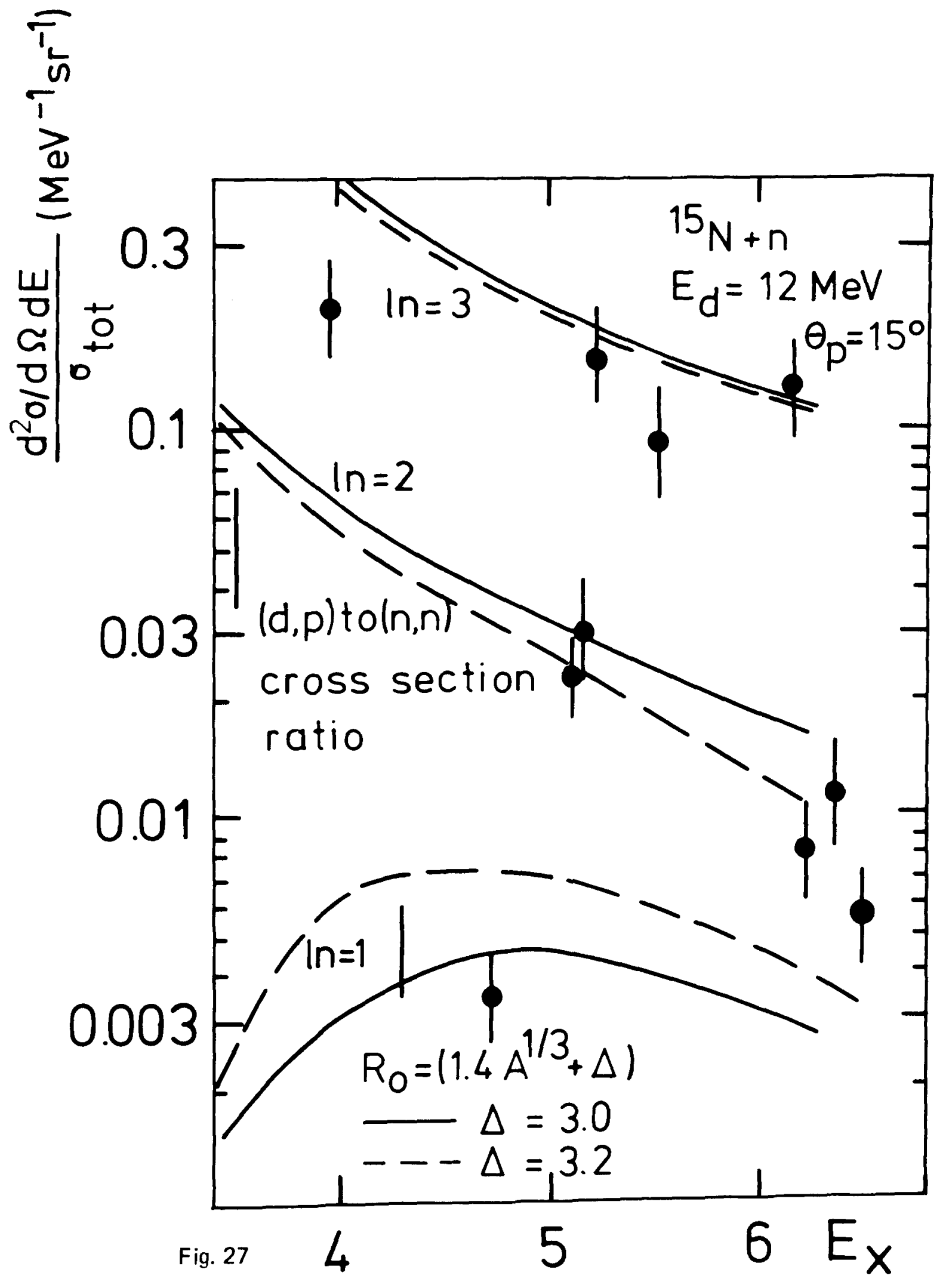




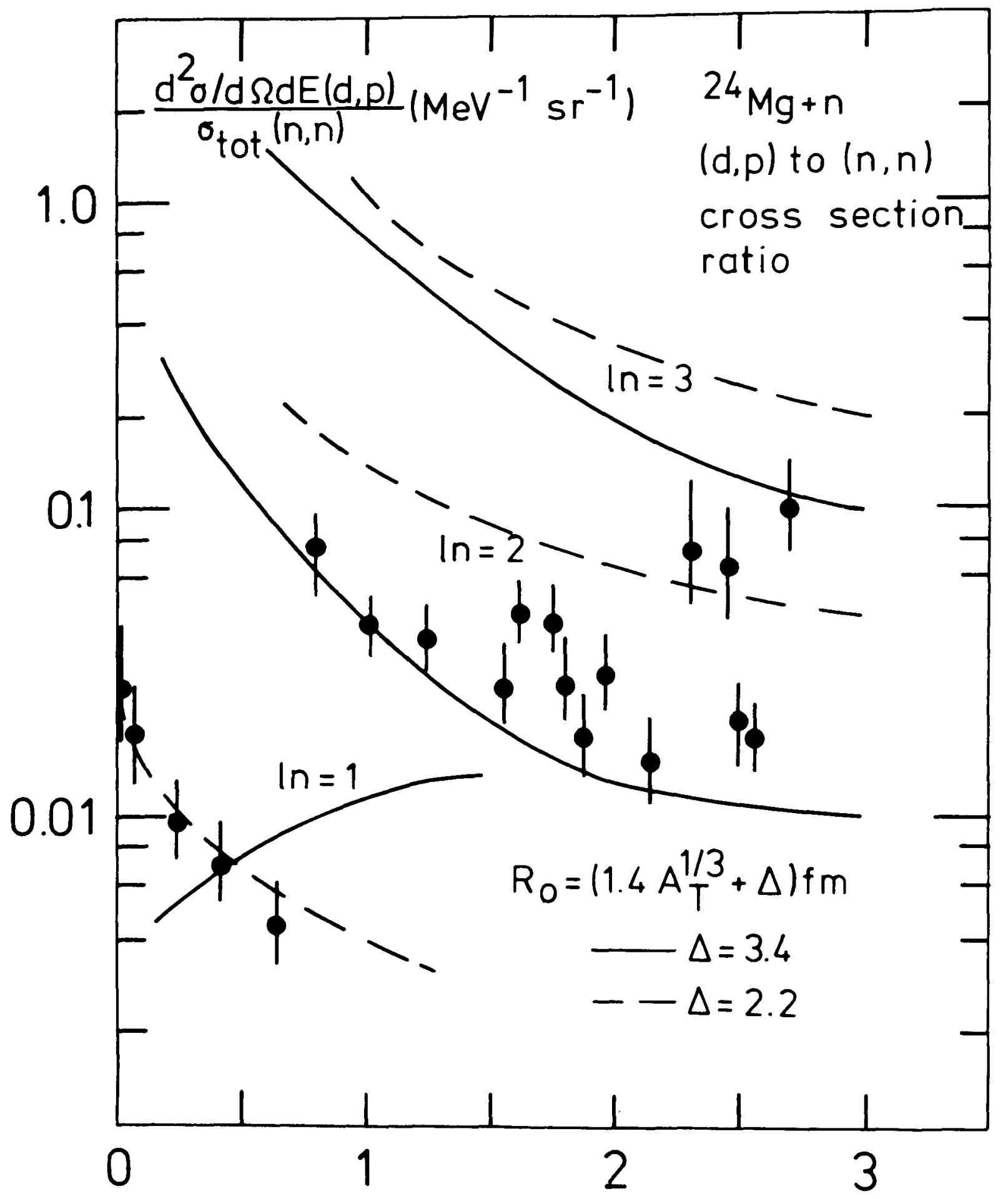

Fig. $28 \quad E_{n}(\mathrm{MeV})$ 

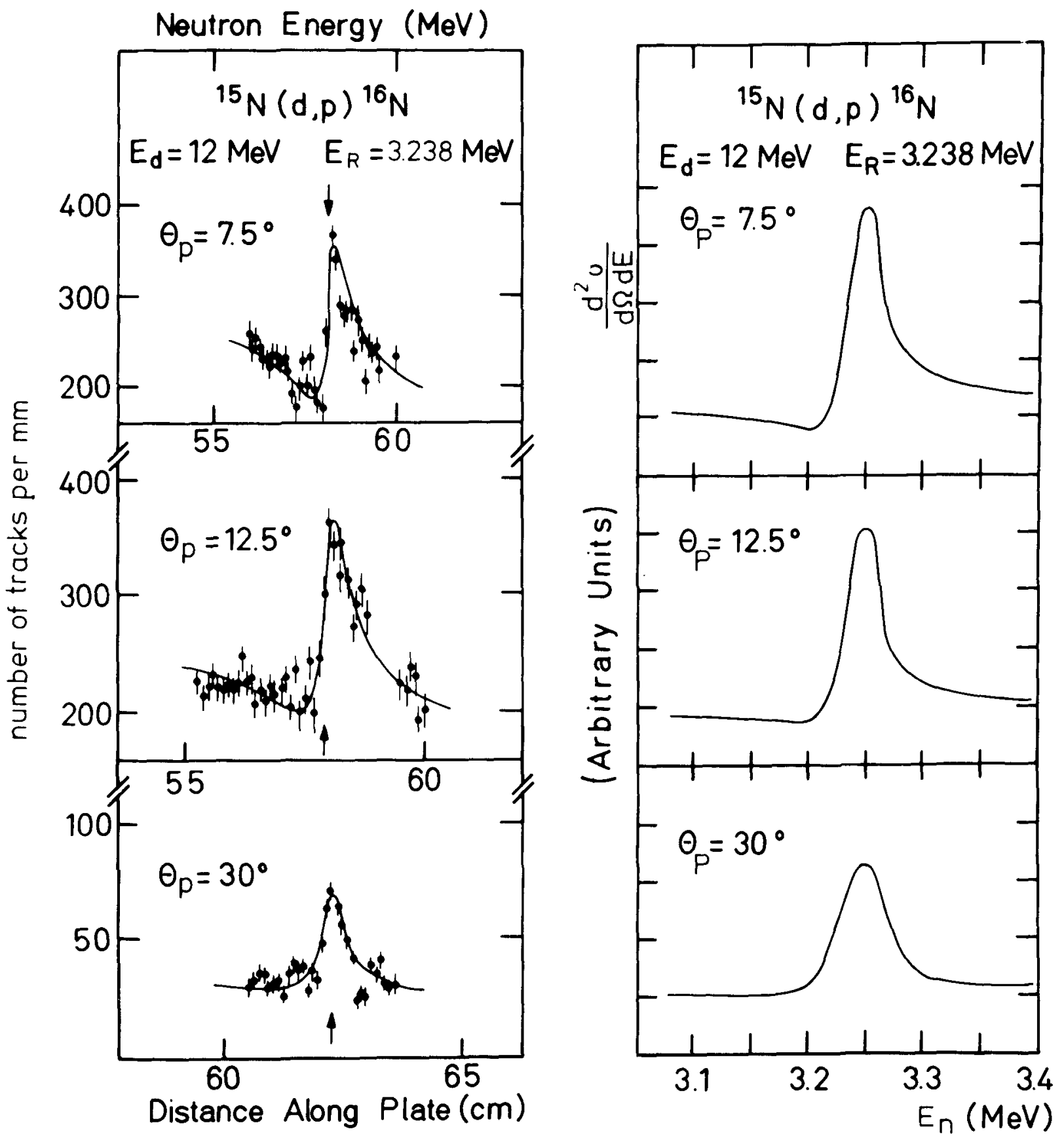

Fig. 29 


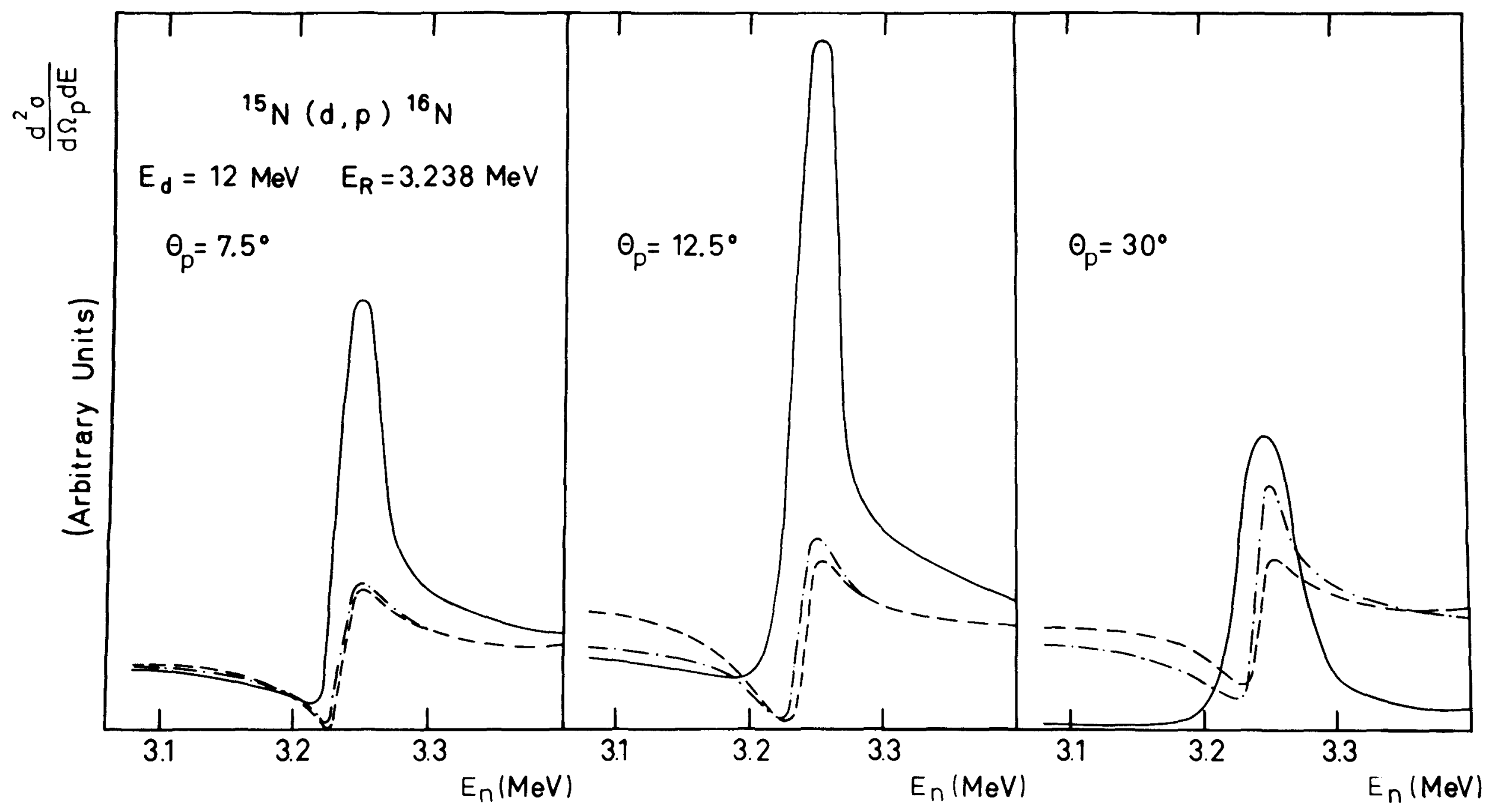

Fig. 30 


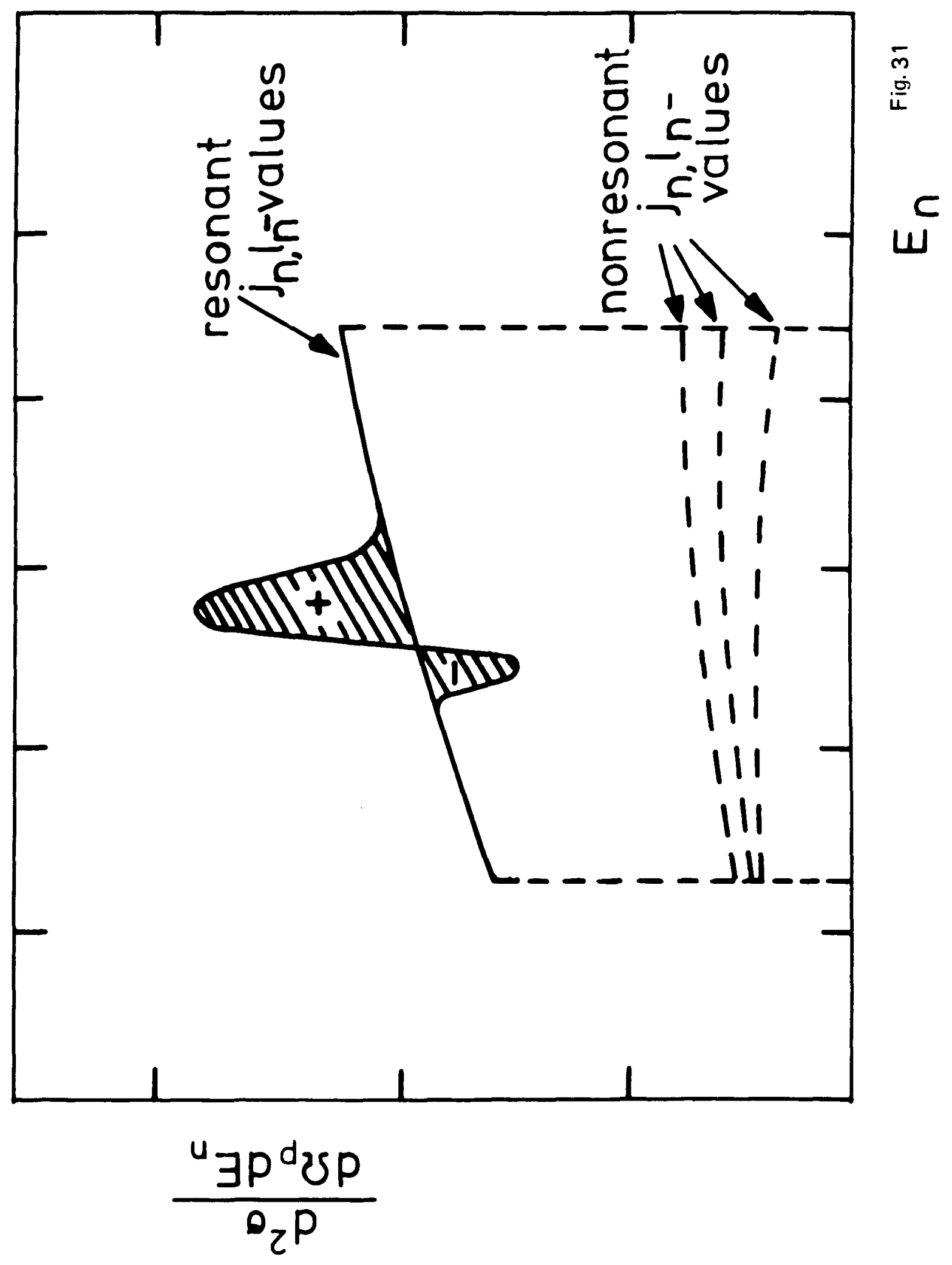




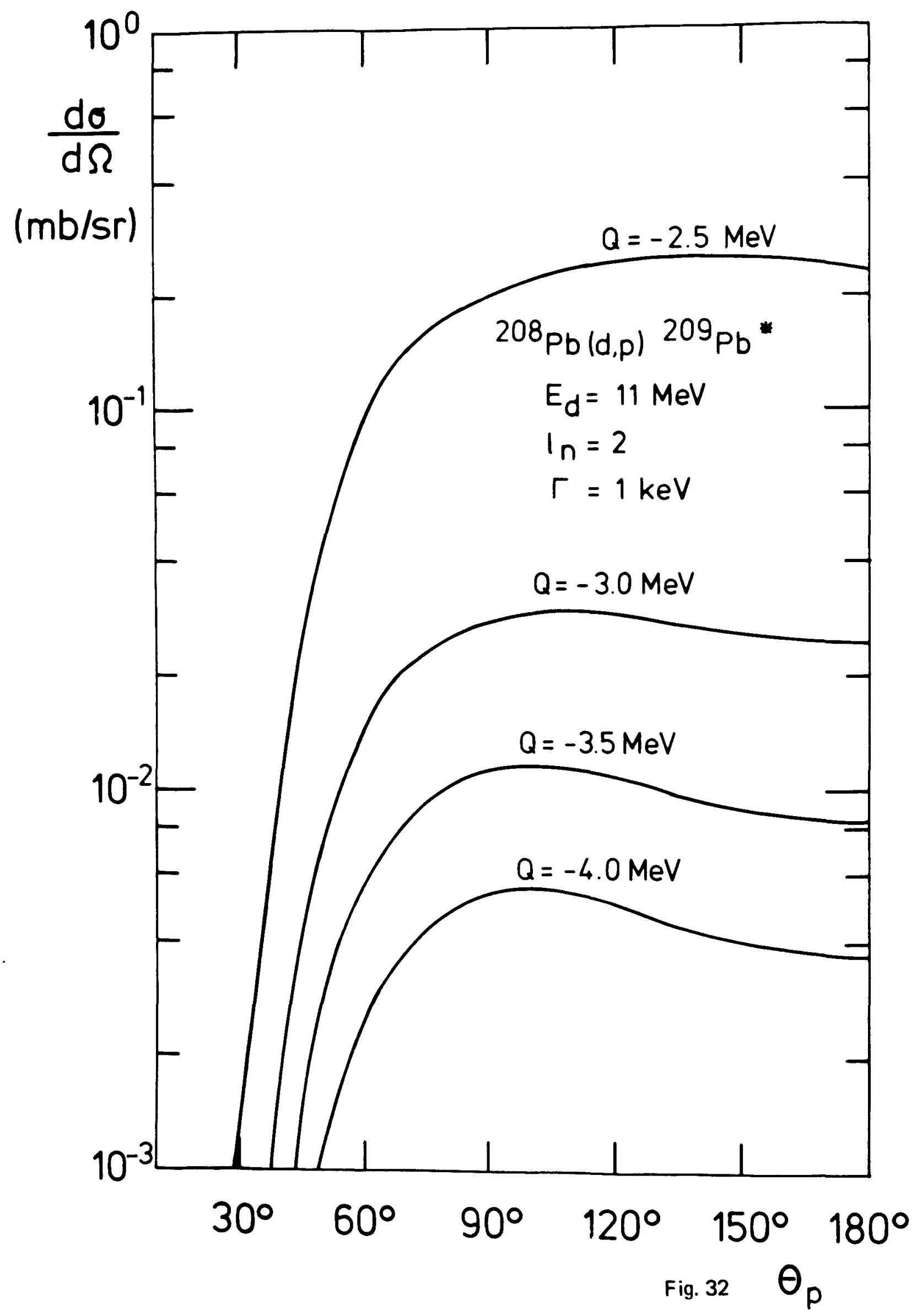




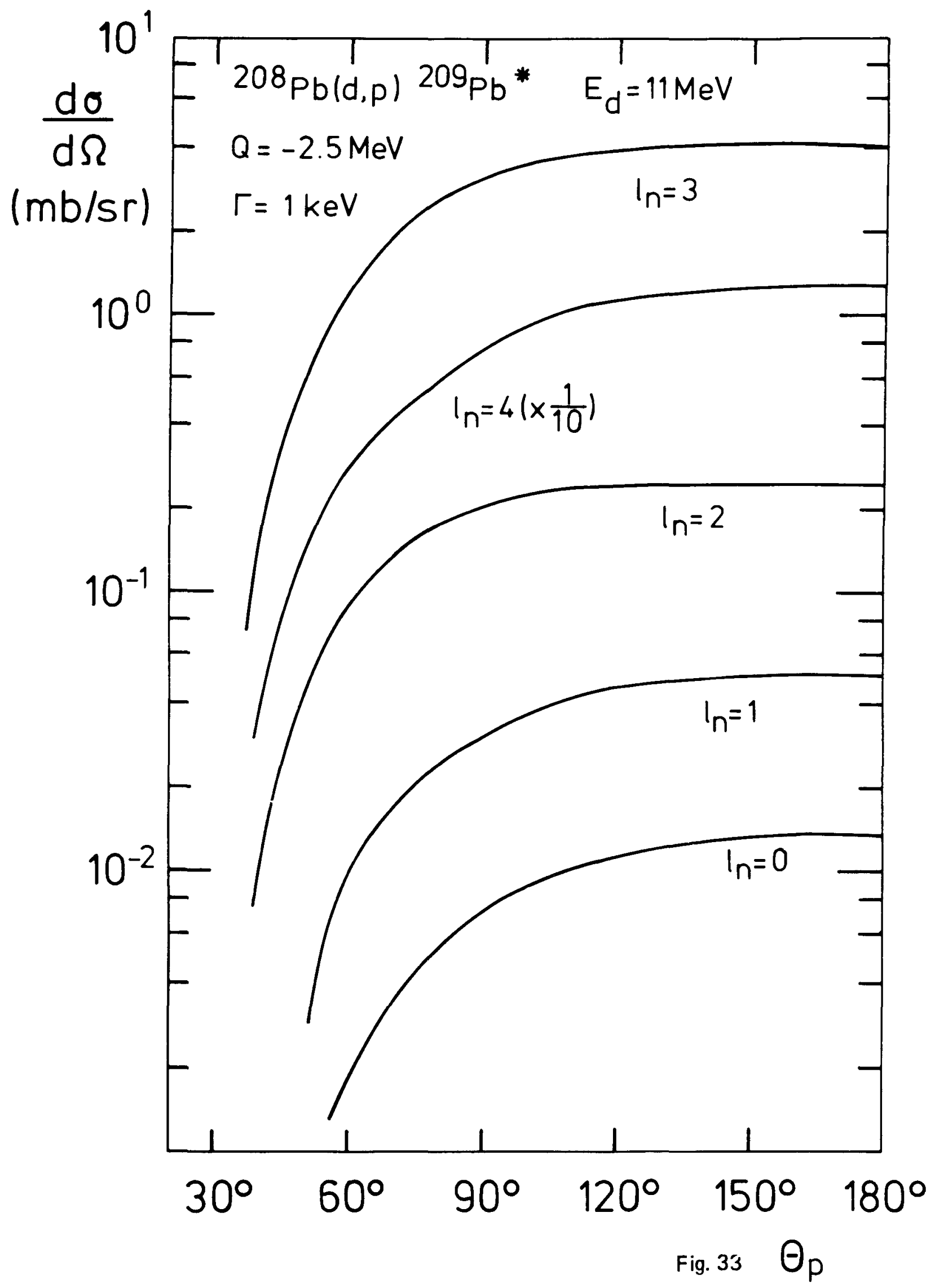




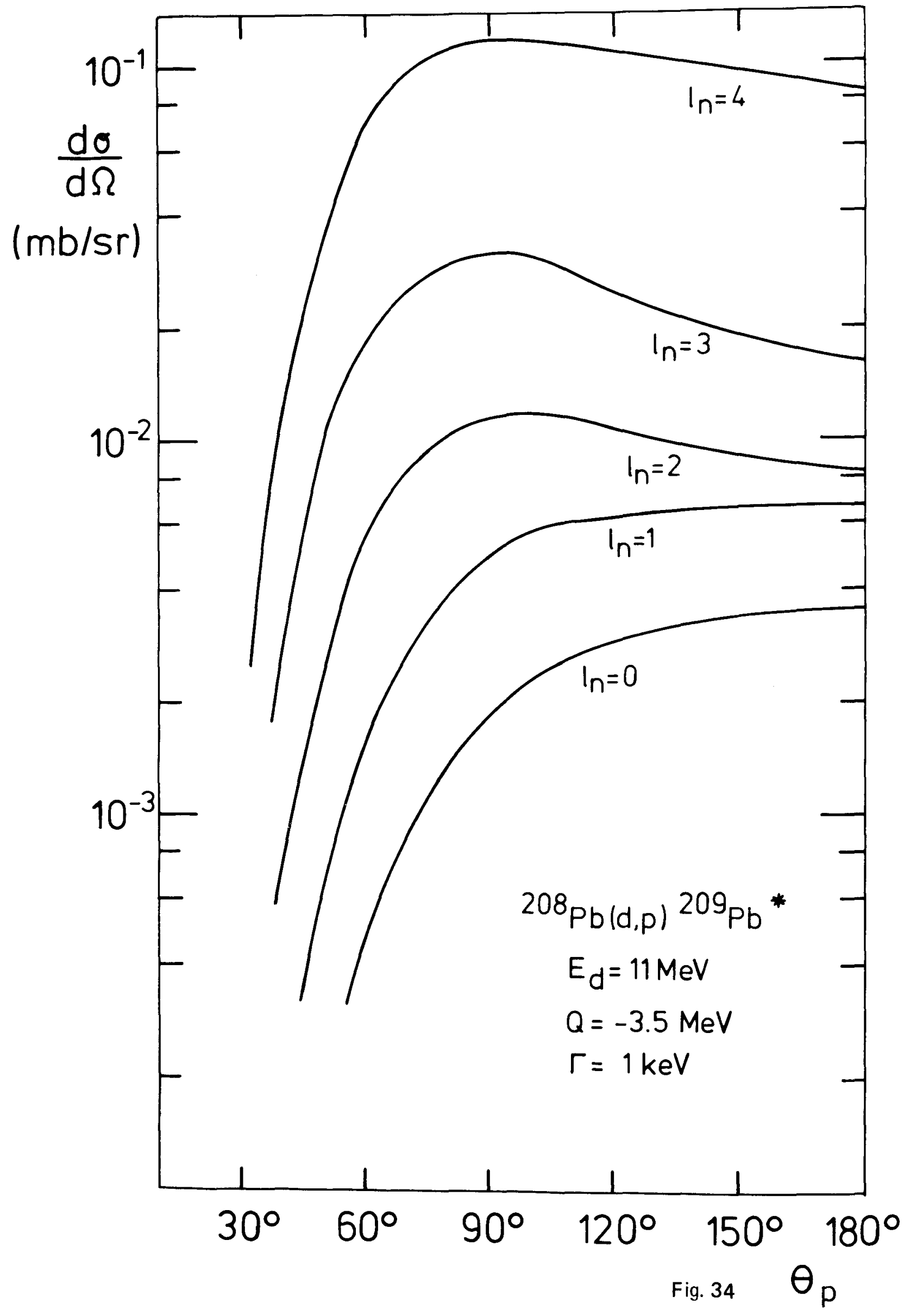




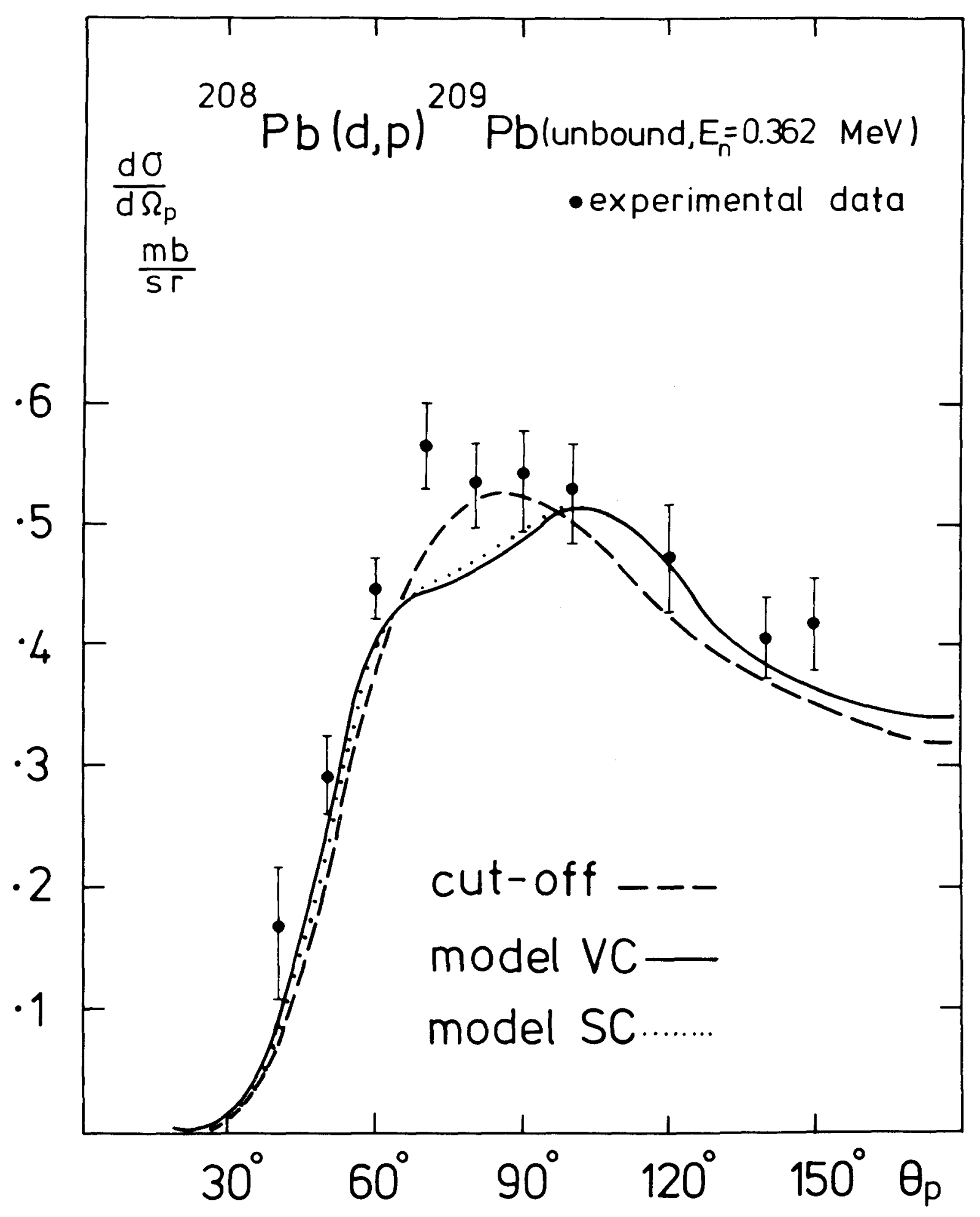

Fig. 35 